\title{
DC2007: 5th International meeting on dendritic cell vaccination and other strategies to tip the balance of the immune system, 16-18 July, 2007, Bamberg, Germany
}

Published online: 31 January 2008

Oral Presentations (16 July 2007)

\section{P001}

CD83 Knockdown in monocyte-derived dendritic cells by Small Interfering RNA (siRNA) leads to a diminished T-cell-stimulation

A. T. Prechtel, N. M. Turza, A. A. Theodoridis, A. Steinkasserer; University Hospital of Erlangen/Department of Dermatology, Erlangen, Germany

Mature human dendritic cells (mDC) are the most powerful antigen-presenting cells known today having the unique ability to induce primary immune responses. One of the best known surface markers for $\mathrm{mDCs}$ is the glycoprotein CD83 that is strongly upregulated during maturation, together with co-stimulatory molecules such as CD80 and CD86. When CD83 surface expression was inhibited by interference with the messenger RNA export or by infection with certain viruses, DCs showed a dramatically reduced capability to induce T-cell proliferation. However, in these cases side effects on other cellular functions cannot be excluded completely.

Here we present an efficient method to specifically influence CD83 surface expression by the use of RNA interference (RNAi). We used small-interfering RNA (siRNA) targeted against CD83 and carefully evaluated an electroporation protocol for the delivery of the duplex into the cells. Furthermore, we identified freshly prepared, immature DCs as the best target for the application of a CD83 knockdown and we were also able to achieve a long lasting silencing-effect for this molecule. Finally, we were able to confirm that CD83 functions as an enhancer during the stimulation of T-cells, significantly increases DC-mediated T-cell proliferation and goes hand in hand with clear changes in cytokine expression during T-cell priming. These results were obtained for the first time without the use of agents that might cause unwanted side effects, such as low molecular weight inhibitors or viruses. Therefore, this method presents a suitable way to influence DCs' biology.

A full meeting report is available at: http://dx.doi.org/10.1007/ s00262-007-0443-8
P002

Potent T-cell mediated antitumor immunity through fully mature, antigen-capturing DCs following vaccination with ligand-loaded CD1d-expressing tumor cells

K. Shimizu ${ }^{1}$, R. M. Steinman ${ }^{2}$, S. Fujiii ${ }^{1}$;

${ }^{1}$ Research Unit for Cellular Immunotherapy, Research Center for Allergy and Immunology, RIKEN, Yokohama, Japan, ${ }^{2}$ Laboratory of Cellular Physiology and Immunology and Christopher H. Browne Center for Immunology and Immune Diseases, The Rockefeller University, New York, NY, USA

NKT cells can link innate and adaptive immunity through the maturation of dendritic cells (DCs) and resultant adjuvant effects. However, NKT cells by themselves after the initial activation by a single dose of $\alpha$-GalCer administration become anergic. In this study, we demonstrate the efficacy of $\alpha$-GalCer-loaded tumor cells (tumor/Gal) in promoting protective immunity, especially in mice given $\alpha$-GalCer-loaded, CDld-highly expressing tumor cells. Immunization with tumor/Gal induced prolonged IFN- $\gamma$ secretion by NKT cells, which are potent mediators of innate immunity. In fact, tumor/Gal, such as B16/Gal-immunized mice survived for a long term, 6-12 months.

At the second step following the lysis of tumor cells by the activated NKT and NK cells, the killed tumor cells are taken up by neighboring DCs. Once neighboring DCs take up apoptotic tumor cells, they simultaneously cross present glycolipid on CD1d to NKT cells, undergo full maturation in vivo, leading to cross-priming of $\mathrm{T}$ cells. We successfully demonstrated the rejection of B16 tumor challenge (s.c) in B16/Gal cell administered mice. Our strategy turned out to be much powerful than the conventional peptide-pulsed DC therapy. The initial magnitude of the innate immune response correlates well with the subsequent induction of adaptive immunity. Thus, vaccination with tumor/Gal leads to the development of specific $T$ cell responses to epitopes present on the unmodified parental tumor cells by antigen-captured, fully matured endogenous DCs and could therefore constitute a potent immunotherapeutic strategy by enhancing both innate and adaptive tumor immunity. 
P003

Tumor endothelium in human pancreatic carcinoma promotes selective accumulation of $\mathrm{CD}^{+} \mathrm{CD}^{+} 5^{+}$ regulatory $\mathbf{T}$ cells

P. Beckhove ${ }^{1}$, D. Nummer ${ }^{1}$, E. Suri-Payer ${ }^{1}$,

H. Schmitz-Winnenthal ${ }^{2}$, A. Bonertz ${ }^{1}$, D. Antolovich ${ }^{2}$, M. Koch ${ }^{2}$, L. Galindo ${ }^{2}$,

M. Buchler ${ }^{2}, J$. Weitz ${ }^{2}$, V. Schirrmacher ${ }^{1}$;

${ }^{1}$ German Cancer Research Center, Heidelberg, Germany,

${ }^{2}$ Department of Visceral Surgery, University Hospital, Heidelberg, Germany

Regulatory T cells (Treg) have been detected in tumor tissues of various human carcinomas and may play a role in preventing spontaneous tumor rejection by the immune system. Using immunohistochemistry on tissue sections as well as flow cytometry of isolated cells, we demonstrate that CD4+, CD25+ and Foxp3+ Treg, selectively accumulate in pancreatic tumor tissue but not in adjacent non malignant pancreas. Absolute and relative Treg numbers were increased within pancreatic carcinomas compared to adjacent pancreas, and the percentage of Treg among CD4 cells was much higher in both tissues compared to blood. In contrast to conventional $\mathrm{T}$ cells, Tregs exerted an increased capacity to adhere to and transmigrate through autologous tumor-derived endothelial cells in vitro and, in an xenotransplant NOD/Scid mouse model, in vivo. Blocking by monoclonal antibodies revealed that this capacity was mediated by interactions of adhesion molecules, including CD62-E, MAdCAM-1, VCAM-1 and CD166 which were differentially overexpressed on tumor-derived endothelial cells compared to conventional endothelial cells with their respective ligands 37 integrin, CD62L and CD166 which were found to be selectively expressed on Tregs. The data provide evidence that tumor induced changes of adhesion molecule expression on endothelial cells allows a selective transmigration of Tregs through tumor endothelium. This mechanism may support the observed selective recruitment of Tregs into human pancreatic carcinomas and opens the possibility of blocking Treg transmigration through Abs against respective adhesion molecules in order to reduce Treg frequencies in tumors as a therapeutic strategie to strengthen anti tumor immune responses.

(J.N.C.I., in press)

\section{P004}

\section{Differentiation of adaptive $\operatorname{Tr} 1$ cells through} tolerogenic DC requires the $\mathrm{IL}-10$-dependent ILT-4/HLA-G pathway

\author{
S. Gregori ${ }^{1}$, V. Pacciani $1^{2}$, D. Tomasoni ${ }^{1}$, M. Battaglial ${ }^{3}$, \\ M. Roncarolo ${ }^{4}$. \\ ${ }^{I}$ San Raffaele Telethon Institute for Gene Therapy (HSR-TIGET), \\ Milan, Italy, ${ }^{2}$ University of Tor Vergata, Rome, Italy, ${ }^{3}$ Immunol- \\ ogy of Diabetes, Milan, Italy, ${ }^{4}$ Vita Salute San Raffaele University, \\ Milan, Italy
}

Interleukin-10 (IL-10) is a potent immunomodulatory cytokine that plays a central role in controlling inflammatory processes, suppressing T-cell responses, and maintaining immunological tolerance. IL-10 is crucial for the induction of adaptive Tr 1 cells, but the molecular mechanism underlying $\operatorname{Tr} 1$ cell differentiation is still unknown. Tr1 are defined by their unique cytokine production profile (i.e. IL-10+ +, IL-5 +, TGF- $\beta+$,IL4-,IL-2low,IFN- $\gamma$ low), are anergic, and down-modulate immune responses through the production of the immunosuppressive cytokines IL-10 and TGF- $\beta$.
We identify a unique subset of dendritic cells (DC), termed DC-10, which are present in vivo, can be induced in vitro in the presence of IL-10, and secrete high levels of IL-10 but low IL-12. DC-10 are characterized by the expression of CD14, CD11c, CD11b, CD83, CD71 and HLA-DR, but not CD1a, display a mature myeloid phenotype, and express immunoglobulin-like transcript (ILT)-2, ILT-3, ILT-4, and HLA-G. DC-10 promote induction of Tr1 cells in vitro through the ILT-4/HLA-G pathway, which is dependent on IL-10. ILT-4/HLA-G interaction is a critical step in the DC-mediated induction of $\operatorname{Tr} 1$ cells. In conclusion, the identification of DC10 and the mechanism underlying $\operatorname{Tr} 1$ cells differentiation is an important step towards the understanding of this regulatory $\mathrm{T}$ cell subset.

\section{P005}

Antibody-mediated targeted delivery of immunomodulatory agents to dendritic cells: proof of concept using the Notch ligand Delta1

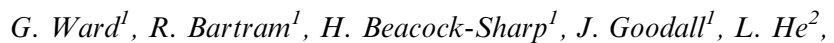
L. Young ${ }^{1}$, T. Keler ${ }^{2}$, B. R. Champion ${ }^{1}$;

${ }^{1}$ Celldex Therapeutics Ltd, Cambridge, UK, ${ }^{2}$ Celldex Therapeutics Inc, Phillipsburg, NJ, USA

Antibody-mediated targeting of antigens to dendritic cells (DCs) and other APC's is a specific and efficient delivery platform for direct in vivo application to the development of vaccines. Recently, the first candidate APC-targeted vaccine, which utilizes the human mannose receptor (hMR) specific fully human antibody, B11, has begun clinical trials for cancer immunotherapy. In principle, such antibodies to dendritic cells could also be used to selectively deliver immunomodulatory agents to APCs, either to modulate their functions directly or to modulate (enhance or suppress) the responses of T-cells interacting with them. However, to date there has been very little exploration of these potential applications.

We have used an anti-hMR antibody (Ab) as a model system to generate data supporting the concept of using APC-targeted delivery of immunomodulators for the induction of antigen-specific immune suppression. As immunomodulatory molecule we used the Notch ligand Delta1, which we have shown can promote regulatory cytokine secretion by T-cells. An Ab-Deltal fusion protein was produced that could effectively bind to both hMR and to Notch, and could activate Notch signalling in a reporter cell assay when bound to hMR, demonstrating bifunctionality of the molecule. It interacted well with DCs and could promote IL-10 secretion by activated T-cells. In vivo studies in hMR transgenic mice, with KLH as a model antigen, showed that Ab-Delta 1 pre-treatment induced a state of hyporesponsiveness to a subsequent strong immunogenic challenge with KLH in CFA. No such activity was seen in wild-type mice, indicating that APC-targeting was required for activity.

\section{Cancer Vaccination}

\section{P006}

A combination of MIP-3 alpha-transfected tumor cell vaccine with dendritic cell-based cancer vaccine enhances protection against tumor challenge

\author{
K. Liu, H. Yang; \\ National Health Research Institutes, Taipei, Taiwan
}

The aim of this study is to evaluate the efficacy of combining tumor cell-based and dendritic cell (DC)-based cancer vaccines. 
MIP- $3 \alpha$ was known to be the most powerful chemokine in inducing migration of immature DCs that express CCR6, the receptor for MIP-3 $\alpha$. We proposed to utilize MIP-3 $\alpha$-transfected tumor cells for in vivo conditioning of vaccination sites and that a sequential vaccination of irradiated MIP-3 $\alpha$-producing tumor cells followed by DCs pulsed with tumor cells can generate a stronger immune response and have a better efficacy in inhibiting the growth of tumor cells. Subcutaneous injection of irradiated tumor cells expressing MIP- $3 \alpha$ should effectively attract immature DCs to the injection site and internalize tumor materials. MIP- $3 \alpha$ secreted at the injection site can create an inflammatory environment that can promote the migration of immature DCs to draining lymph nodes for optimal activation of $\mathrm{T}$ cells. This inflammatory environment can further enhance the maturation and activation of the subsequently injected tumor-pulsed DCs as a DC-based cancer vaccine. Our results indicated that s.c. injection of mice with irradiated tumor cells expressing MIP- $3 \alpha$ only marginally inhibited tumor growth. However, vaccination of irradiated tumor cells expressing MIP-3 $\alpha$ followed by DC pulsed with tumor cells can effectively protect animals from tumor challenge. The protection effect was better when the tumor cell-based and DC-based vaccines were given at the same site and in such order. These results confirm that such a combinational vaccination strategy may generate a more effective immune response to suppress the growth of tumor cells.

\section{P007 \\ Identification and characterization of $T$ cell epitopes deduced from RGS5, a novel broadly expressed tumor antigen}

C. N. Bo $\boldsymbol{\beta}^{1}$, F. Grünebach ${ }^{1}$, K. M. Brauer ${ }^{1}$, T. Weinschenk ${ }^{2}$, L. Kanz ${ }^{1}$, S. Stevanovic ${ }^{3}$, H. Rammensee ${ }^{3}$, P. Brossart ${ }^{1}$;

${ }^{I}$ Abteilung für Hämatologie, Onkologie, Immunologie, Rheumatologie und Pulmologie, Universitätsklinikum Tübingen, Tübingen, Germany, ${ }^{2}$ immatics biotechnologies GmbH, Tübingen, Germany, ${ }^{3}$ Institut für Zellbiologie, Abteilung für Immunologie,

Tübingen, Germany

Using a comparative DNA chip analysis of renal cell carcinoma (RCC) and corresponding non-malignant tissue two HLA-class I bound peptides derived from the RGS5 protein were identified. RGS5 was previously shown to be expressed in pericytes and promote tumor angiogenesis. Using RT-PCR analysis we found that RGS5 is also expressed on a broad variety of solid tumors and leukemias and markedly overexpressed in 16 of 29 analyzed primary RCC samples, making it an interesting candidate for the development of vaccination strategies to target tumor cells and tumor vessels. To confirm the immunogenicity of identified epitopes cytotoxic $\mathrm{T}$ lymphocytes (CTL) were generated using dendritic cells (DC). CTL induced using the RGS5 peptides lysed autologous DC pulsed with the cognate peptide or transfected with in vitro-transcribed RGS5-RNA as well as HLA-matched tumor cell lines. The specificity and HLA restriction was confirmed using blocking antibodies and coldtarget inhibition assays. Processing and presentation of the identified peptides by malignant cells were confirmed using DC transfected with RGS5-RNA to generate specific CTL. These CTL lysed tumor cells in an antigen specific manner while sparing non-malignant cells. To analyze the induction of RGS5 specific CTL in an autologous setting we used blood samples from a patient with AML and were able to generate specific CTL capable of recognizing autologous AML blasts while sparing non-malignant cells.
Our results demonstrate that RGS5 is a novel tumor rejection antigen expressed in a wide range of malignancies that can be applied to target malignant cells and tumor angiogenesis.

\section{P008}

Integration of DC vaccination in the primary treatment of patients with GBM: preliminary experiences in adult patients

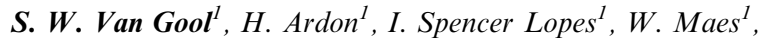

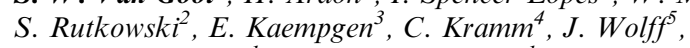 \\ F. Van Calenbergh ${ }^{1}$, S. De Vleeschouwer ${ }^{1}$; \\ ${ }^{1}$ Catholic University of Leuven, Leuven, Belgium, \\ ${ }^{2}$ University of Wuerzburg, Wuerzburg, Germany, \\ ${ }^{3}$ University of Erlangen, Erlangen, Germany, ${ }^{4}$ University of Halle, \\ Halle, Germany, ${ }^{5}$ MD Anderson Cancer Center, Houston, TX, USA
}

Purpose: In spite of neurosurgery, radiochemotherapy (RCT) and maintenance temozolomide chemotherapy (TZMm), the prognosis of patients with GBM remains poor. We integrated immunotherapy in the primary treatment for adults with GBM as phase I/ II protocol HGG-2006.

Methods: After (sub)total resection, leukapheresis was performed prior to, and 4 weekly vaccinations with autologous GBM lysateloaded DC were given after RCT. Boosts with GBM lysates were given during TMZm.

Results: Twenty-four patients (median age 52 years, 17-66) were treated. The proportion without progression at $6 \mathrm{~m}$ was $75.3 \%$. The OS is $100 \%$, with median FU of $5.3 \mathrm{~m}$ (1.6-22). Three patients were slightly progressive already during radiochemotherapy but could still be treated. Immune phenotyping showed an increase of the HLA-DR + fraction in the T cell population and the $\mathrm{CD} 25+$ fraction in CD4 + and CD8 $+\mathrm{T}$ cells after RCT. After 4 weekly DC injections, a further increase of the CD25 + fraction in the $\mathrm{CD} 8+\mathrm{T}$ cell population was observed. An increase of the frequency of tumor antigen-reacting IFN- $\gamma$-producing $\mathrm{T}$ cells was found with ELISPOT at time of vaccine 4 and 7 , in comparison to the two samples prior to vaccination, in $4 / 8$ tested patients. In one patient a sudden onset hemiplegia and aphasia was diagnosed as ischemic event at 8 months follow-up. Inflammation could not be ruled out. Quality of life during vaccination remained excellent. Conclusion: Tumor vaccination integrated within the standard primary postoperative treatment for patients with newly diagnosed malignant glioma is feasible, well tolerated and possibly beneficial for patients with minimal residual tumor burden.

\section{P009}

Therapeutic dendritic cell vaccination with ag coupled to cholera toxin in combination with intratumoural CpG injection leads to complete tumour eradication in mice bearing HPV 16 expressing tumours M. L. Nurkkala ${ }^{1}$, A. George Chandy ${ }^{1}$, A. Josefsson

We have evaluated whether cholera toxin (CT) can enhance the efficiency of therapeutic dendritic cell (DC) vaccination in mice bearing a human papilloma virus (HPV) 16 antigen (Ag) expressing tumour. Mice were therefore injected with the TC-1 cancer cell line expressing E6 and E7, which are the major oncogenic proteins produced in HPV induced cervical cancer, and 
they were then vaccinated with Ag pulsed DCs. While vaccination with E7 pulsed DCs had no impact on tumour growth, DCs pulsed with CT conjugated E7 (CT-E7) significantly reduced tumour size. However, this treatment was only able to eradicate the tumour in $11 \%$ of the affected animals. For complete tumour eradication, combinational therapy with CT-E7 pulsed DCs and local treatment of the tumour with $\mathrm{CpG}$ was required. Combinational therapy was associated with increased expression of MHC I and MHC II and increased levels of chemokine production in the tumour. These results suggest that combined treatment with CT-Ag pulsed DCs and local CpG administration offers an efficient strategy to eradicate an already existing HPV-E7 expressing tumour in mice.

\section{P010 \\ Age-dependent dendritic cell function: implication for cancer vaccine therapy}

\section{A. Grolleau-Julius, E. Harning, R. Yung; \\ University of Michigan, Ann Arbor, MI, USA}

We recently reported that bone marrow-derived dendritic cells (DCs) from old $\mathrm{C} 57 \mathrm{BL} / 6$ mice are less effective than their young counterpart in inducing the regression of pre-established B16-OVA melanomas. To examine the underlying mechanisms, we investigated the effect of aging on DC tumor antigen presentation and migration.

Although aging does not appear to affect the ability to present OVA peptide (SIINFELK) in the context of MHC class I molecule, DCs from aged (18-20 months) C57BL/6 mice are less efficient than those from young (3-6 months) C57BL/6 mice to stimulate MHC class I OVA-specific T cell in vitro $(P<0.0025)$ and in vivo $(P<0.025)$. This in turn correlates to a $43 \%$ reduction in the number of intra-tumor CD8 $\mathrm{T}$ cells in mice receiving OVA peptide-pulsed old DCs. The defect in T cell stimulation may be linked to the reduced DC-SIGN expression in aged DCs. To examine in vivo DC trafficking, we injected young and old fluorescent-labeled DCs into the footpad of $\mathrm{C} 57 \mathrm{BL} / 6$ mice, and quantified their migration to the lymph nodes by fluorescent microscopy. Half as many aged DCs were found to be present in the popliteal lymph nodes $(P<0.025)$. This correlates with reduced CCR7 gene expression $(P<0.0005)$, impaired in vitro migration of aged DCs in response to CCL21 $(P<0.05)$, and defective CCR7 signaling in aged DCs.

Taken together, our findings suggest that defective DC migration and $\mathrm{T}$ cell stimulation contribute to the observed impaired DC tumor immunotherapeutic response in aging.

\section{P011 \\ In vivo anti-cancer effect of dendritic cell vaccine in mouse leukemia model : comparing the effect of CD40L vs. PGE2 maturation}

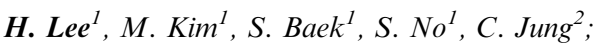 \\ ${ }^{1}$ The Cancer Center, Samsung Medical Center, Seoul, \\ Republic of Korea, ${ }^{2}$ Department of Hematology/Oncology, \\ Samsung Medical Center, Seoul, Republic of Korea
}

In this study, the differential effects of CD40L and PGE2 as maturation factor for anti-cancer therapeutic-DC were observed. Balb/ c syngeneic leukemia cell line, WEHI-3 was inoculated intravenously. Monocyte-derived DCs were cultured from mouse bone marrow cells with GM-CSF and IL-4 (1,000 IU/ml each). After 6 days, WEHI-3 lysate pulsed DCs were matured with CD40L or PGE2 for $18 \mathrm{~h}$. Therapeutic DCs were harvested and injected twice into the peritoneum with one week interval from one day after the tumor cell injection. Cultured DCs were characterized by phenotype analysis and cytokine production. After 2 weeks from the last DC injection, tumor bearers were sacrificed to monitor the tumor-specific immune induction. Tumor formation in the lung was measured as therapeutic effect of DC vaccination as well. The cultured cells confirmed as DCs by phenotype analysis. While IL-10 secretion was higher in PGE2-DC (424.7 vs. $284.6 \mathrm{pg} / \mathrm{ml}$ for PGE2-DC vs. CD40L-DC, respectively), IL-12 secretion was higher in CD40LDC (0.42 vs. $21.72 \mathrm{pg} / \mathrm{ml}$ for PGE2-DC vs. CD40L-DC, respectively). Auto MLR induced by CD40L-DC was higher than that of immature-DC or PGE2-DC. In the DC vaccine treated tumor bearing mice, tumor-specific $\mathrm{T}$ cell proliferations and the number of IFN-r secreting CD8 $+\mathrm{T}$ cells were highest in the CD40L-DC group (257, 113 and 1735 spots $/ 10^{5} \mathrm{CD} 8+$ cells for saline, PGE2DC and CD40L-DC group, respectively). Based on the tumorspecific immune induction and the inhibition of pulmonary tumor formation, CD40L-DC might be the better therapeutic module than PGE2 matured DCs in anti-cancer DC vaccine.

\section{P012}

\section{Experimental background and experience of clinical use of dendritic-cell vaccines in complex therapy of advanced cancer patients}

\author{
N. M. Khranovska, V. E. Orel, N. P. Tsyp, O. V. Skachkova; \\ Institute of Oncology AMS of Ukraine, Kyiv, Ukraine
}

The objective of study: To determine the most effective ways of generation and application of dendritic-cell vaccines in experiment, to study direct and immediate immunologic and clinical results of their use in complex treatment of cancer patients.

Materials and methods: Splenic DC at amount of $2 \times 10^{5} /$ mouse loaded with lysate of tumor cells (LTC) or mechanomodified lyophilized tumor cells (MMLTC) were infused to CBA or C57Bl/ 6 mice 3 days after inoculation of sarcoma 37 (S37) or removal of primary tumor (Lewis' lung carcinoma, LC). Ways of administration - intracutaneous, subcutaneous, intraperitoneal or intravenous. 59 lung cancer (LC) IIIA stage patients and 55 advanced ovarian cancer (AOC) IIIc-IV stages patients were infused postoperatively by $4,62 \pm 0,48 \times 10^{6}$ of DC loaded with MMLTC or LTC intravenously (2-11 infusions).

Results: The most effective ways of DC-vaccines are: three times with 3-7 days interval intravenously (DC loaded with LTC S37, ratio $1 \mathrm{DC}: 3$ tumor cells, $10^{7}$ cells $/ \mathrm{ml}$ or loaded with MMLTC LTC LC, $0.05 \mathrm{mg} / \mathrm{ml}$, in mouse with S37 and LC, respectively). DC- vaccinotherapy was accompanied by increase of amount of CD8, CD16-lymphocytes, activated lymphocytes (HLA-DR+, $\mathrm{CD} 38+, \mathrm{CD} 71+)$ and functional activity of NK-cells. The survival of AOC patients who received DC-vaccinotherapy within a year was $89 \%$, and in group of comparison - $81 \%$, LC patients $82 \%$ versus $65 \%$. Two-year survival of AOC patients was 46 and $10 \%$, respectively to groups with/without DC-vaccinotherapy $P=0.01$ ).

Conclusion: Thus, DC-vaccinotherapy in complex treatment of AOC and LC patients is encouraging. 


\section{P013}

Immunotherapy with irradiated autologous leukaemic cells in patients with B-CLL in early stages

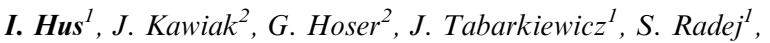 \\ A. Dmoszynska ${ }^{1}, J$. Rolinski ${ }^{1}$; \\ ${ }^{1}$ Medical University of Lublin, Lublin, Poland, ${ }^{2}$ Medical Centre of \\ Postgraduate Education, Warsaw, Poland
}

The assumption of the study was based on the hypothesis, that stimulation of immature dendritic cells, such as skin Langerhans cells by tumour antigens released from apoptotic leukemic cells, and then processing and presenting of these antigens to $T$ cells in regional lymph nodes would lead to activation of specific CTL against B-CLL cells. For this, peripheral blood mononuclear cells including on average $78.56 \%$ of $\mathrm{CD} 19+/ \mathrm{CD} 5+$ lymphocytes were irradiated, and then administered intradermally to 17 patients with B-CLL in early stages. In 8 out of 17 patients, in the first two injections, irradiated leukemic cells were mixed with BCG with intend to improve the efficacy of therapy by additional induction of innate immunity. A clinical response $(>25 \%$ reduction of $\mathrm{WBC}$ count) was observed in $5 / 17$ patients, stabilisation of disease also in $5 / 17$ patients and in $7 / 17$ patients there was no response to immunotherapy. In seven patients significant increase of lymphocytes doubling time was noted $(24.00 \pm 16.97$ vs. $9.28 \pm 5.65$ months; $P=0.02$ ). There was no impact of $\mathrm{BCG}$ for either immune responses or clinical outcome of vaccinated patients. In the whole patients' group there was a significant increase of $\mathrm{CD} 3+\mathrm{T}$ cells absolute counts during vaccination period. Before the start and during immunotherapy, percentages of $\mathrm{CD} 3+\mathrm{T}$ cells and $\mathrm{NK}$ cells were significantly higher in responding patients. In conclusion, our results demonstrated feasibility and safety of autologous irradiated leukemic cells immunotherapy in patients with B-CLL and suggest further investigations with regard to modifications aimed for improvement of the results of therapy.

\section{P014}

In vivo efficacy of hematopoietic stem cell-derived allogeneic-DC vaccine in mouse melanoma metastasis model

\author{
H. Lee ${ }^{1,2}$, M. Kim ${ }^{1}$, S. Baek ${ }^{1}$; \\ ${ }^{1}$ The Cancer Center, Samsung Medical Center, Seoul, Republic \\ of Korea, ${ }^{2}$ Sungkyunkwan Univ., Sch. of Medicine, Seoul, \\ Republic of Korea
}

Dendritic cell (DC) based cancer vaccine is being studied for several years with cells mainly derived from autologous PBMC of patients. Autologous-DC culture protocol has limitations in yield and ability of DC differentiation based on patient's individual status. In order to overcome these limitations, allogeneic-DCs were cultured as therapeutic module but there are not many preclinical answers for effect or toxicity of allogeneic-DCs to use for clinical trial. In this study, we compared the anti-tumor effect of allogeneic and autologous dendritic cells differentiated form mouse bone marrow stem cells in metastatic melanoma model. B16F10 melanoma cells $\left(5 \times 10^{4} /\right.$ mouse $)$ were injected intravenously into the C57BL $/ 6$ mice. Therapeutic DCs differentiated from autologous (C57BL/6:CDC) or allogeneic (B6C3F1:BDC) bone marrow stem cells with GM-CSF, SCF and IL-4 for 13 days and pulsed with B16F10 tumor cell lysate (Blys-DC) for $18 \mathrm{~h}$. Cultured cells were characterized as DCs by phenotype analysis and cytokine secretion assay. Therapeutic-DCs were injected in- tra-peritoneal, twice with 1-week interval. Anti-tumor response was observed by DC vaccine without any toxicity in allogeneicDC treated mice (tumor burden score: $2.667 \pm 0.184$, $2.500 \pm 0.463,2.000 \pm 0.286,1.500 \pm 0.286,1.667 \pm 0.297$ for saline, CDC/Unpulsed-DC:U-DC, CDC/Blys-DC, BDC/U-DC and $\mathrm{BDC} / \mathrm{Blys}-\mathrm{DC}$, respectively). IFN- $\gamma$ secretion was significantly increased in allogeneic-DC group stimulated with B16F10 cell lysate $(2643.3 \pm 589.7,8561.5 \pm 2204.9 .6901 .2 \pm 141.1 \mathrm{pg} /$ $1 \times 10^{6}$ cells for saline, BDC/U-DC and BDC/Blys-DC, respectively) with increased NK cell activity. Conclusively, promising pre-clinical data were obtained that allogeneic-DC can be used for DC-based cancer immunotherapy.

\section{P015}

Tumor immunotherapy by epicutaneous immunization requires Langerhans cells

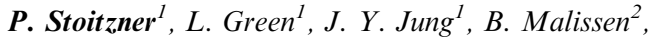 \\ A. Kissenpfennig ${ }^{3}$, I. Hermans ${ }^{l}, F$. Ronchese ${ }^{1}$; \\ ${ }^{1}$ Malaghan Institute of Medical Research, Wellington, \\ New Zealand, ${ }^{2}$ Centre d'Immunologie de Marseille-Luminy, \\ Marseille, France, ${ }^{3}$ Centre of Cancer Research and Cell biology, \\ Belfast, $U K$
}

A role for Langerhans cells (LC) in the induction of immune responses in the skin has yet to be conclusively demonstrated. We used a protocol of skin immunization with ovalbumin protein (OVA) to induce immune responses against OVA-expressing melanoma cells. Mice injected with OVA-specific CD8 $+\mathrm{T}$ cells and immunized with OVA onto barrier-disrupted skin had increased numbers of CD8 $+\mathrm{T}$ cells in the blood, efficiently killed OVA-coated targets in vivo, and their CD8 $+\mathrm{T}$ cells produced IFN-gamma when restimulated in vitro. These mice also generated accelerated cytotoxic responses after secondary immunization with OVA. In contrast, mice immunized with OVA on intact skin showed minor expansion of specific $\mathrm{T}$ cells, low level cytotoxicity, and no IFN-gamma production. Prophylactic or therapeutic immunization with OVA onto barrier-disrupted skin prevented or delayed the growth of B16.OVA tumors, respectively. Delayed tumor growth was also observed in mice not injected with transgenic CD8 $+\mathrm{T}$ cells, and was further enhanced by the TLR7 ligand imiquimod. Depletion of LC at the time of skin immunization dramatically reduced the tumor protective effect, indicating a major role of LC in this immunization protocol. Thus, targeting of tumor antigens to LC in vivo is an effective strategy for tumor immunotherapy.

\section{P016}

A mouse model for glioma immunotherapy with tumor RNA-loaded DC: proof of a tumor-specific immune response in a preventive vaccination strategy

\author{
W. R. Maes, C. M. Deroose, R. Gijsbers, Z. Debyser, \\ J. L. Ceuppens, S. W. Van Gool; \\ Catholic University, Leuven, Belgium
}

We previously reported in a human in vitro setting the feasibility of generating antitumoral CTL through stimulation with autologous DC loaded with total RNA from human glioma cell lines. Recently, we validated the therapeutic efficacy of RNAloaded DC vaccination in a BL6-GL261 mouse glioma model. We also developed a quantitative and non-invasive biolumi- 
nescent in vivo imaging (BLI) technique to evaluate orthotopic tumor growth using GL261 glioma cells lentivirally transduced with luciferase.

Briefly, mice were vaccinated twice intraperitoneally with one million mature bone-marrow derived DC (electroporated with total tumor RNA) before subsequent challenge with GL261Fluc glioma cells. We observed a significant prolonged survival of the mice treated with DC loaded with GL261 total RNA compared to mock or non-vaccinated mice. There was a clear correlation between the intracranial BLI signal and the clinical status of the animals.

To evaluate tumor-specificity of the induced immune response, we isolated spleen and lymph node cells from vaccinated mice with/without subsequent tumor challenge and performed in vitro cytotoxicity assays with these cells. Preliminary results show specific GL261 tumor cell lysis (but no lysis of irrelevant target cells) when lymph node cells from mice vaccinated with RNA loaded DC are used. In vivo we confirmed this tumor-specificity using a subcutaneous tumor model in which mice were vaccinated with DC loaded with GL261 RNA and subsequently challenged with GL261 glioma or MC17-51 fibrosaroma cells. No vaccination effect was seen on fibrosarcoma tumor formation, whereas the growth of GL261 tumors was clearly delayed.

\section{P017 \\ Electroporation of Elutra ${ }^{\mathrm{TM}}$ generated dendritic cells with RNA: towards a closed system}

J. Dörrie, N. Schaft, M. Erdmann, I. Müller, D. Stoica, U. Hirsch, E. Kämpgen, B. Schuler-Thurner, G. Schuler;

University Hospital Erlangen, Department of Dermatology, Erlangen, Germany

Electroporation of monocyte-derived dendritic cells (moDC) with RNA, to load them with antigen, or to manipulate their function, has become a widely used method, also in clinical applications. Since the production of moDC is increasingly performed under GMP conditions, the development of a closed system should prove very beneficial. This is hampered by the standard isolation method of monocytes via plastic adherence from density-gradient purified peripheral blood mononuclear cells. An alternative way to isolate monocytes is cell elutriation via Elutra ${ }^{\mathrm{TM}}$ cell separator, which is a functionally closed system. Here we examined the question whether Elutra ${ }^{\mathrm{TM}}$-generated moDC can be electroporated with equal efficiency and display equal functionality compared to moDC obtained via plastic adherence. Both DC populations were compared with respect to several features relevant for production and effectivity of a DC vaccine after transfection of RNA encoding a tumor antigen. The Elutra ${ }^{\mathrm{TM}}$-derived DC were clearly superior in yield, i.e. a significantly higher amount of DC could be generated from the same amount of leukapheresis product. Both populations could be electroporated with similar, high efficiency (on average $>85 \%$ ), and appeared equal in antigen expression kinetics, antigen-specific $\mathrm{T}$ cell stimulation capacity, CCR7-mediated migration, and expression of surface maturation markers. The data obtained allow for the use of ELUTRAgenerated DC for RNA-electroporation in clinical settings and pave the way for a future fully closed system.

(JD, NS, and ME contributed equally)
P018

\section{Identification of tumor antigens using antibodies induced by DC-based vaccines}

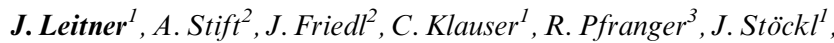 \\ O. Majdic ${ }^{l}$, G. Zlabinger ${ }^{1}, P$. Steinberger ${ }^{1}, K$. Pfistershammer ${ }^{4}$; \\ ${ }^{1}$ Institute of Immunology, Center for Hygiene and Medical \\ Microbiology, Medical University of Vienna, Vienna, Austria, \\ ${ }^{2}$ Department of Surgery, Medical University of Vienna, Vienna, \\ Austria, ${ }^{3}$ Department of Pathophysiology, Medical University of \\ Graz, Graz, Austria, ${ }^{4}$ Department of Dermatology, Division of \\ Immunology, Allergy and Infectious Diseases, Medical University \\ of Vienna, Vienna, Austria
}

Immunizing tumor patients with tumor lysate pulsed dendritic cells (DC) is a promising strategy to elicit efficient anti-tumor response. Numerous studies have demonstrated enhancement of cellular and humoral immune responses after vaccination.

In this study we will focus on patients suffering from medullary thyroid carcinoma who have been immunized with autologous DC loaded with the medullary thyroid carcinoma cell line (MTC). Our aim is to identify and characterize antigens, which are recognized by antibodies induced during this treatment and to analyze the humoral immune response in such patients. We found that several DC-vaccinated patients had a strong antibody response to the surface of the MTC cell line. To identify these antigens we will generate a retroviral cDNA expression library from the MTC tumor cell line and screen it with sera from immunized patients. These molecules should be analzyed regarding $\mathrm{T}$ cell responses and expression in primary tumors.

Identification of such tumor antigens could eventually lead to improved DC-vaccination strategies in medullary thyroid carcinoma based on defined antigens and could also be useful to monitor the success of the immunotherapy. Finally as our approach aims to identify surface tumor antigens this study could lead to novel therapeutic strategies.

\section{P019}

\section{Mammalian cells under pressure: cell death pathways and immunogenicity of dying cells}

\author{
C. Janko ${ }^{1}$, U. Gaipl ${ }^{1}$, S. Franz ${ }^{1}$, N. Ebel ${ }^{2}$, E. Schlücker ${ }^{2}$, \\ R. Meyer-Pittroff ${ }^{3}$, M. Herrmann ${ }^{1}$, B. Frey ${ }^{4,1}$; \\ ${ }^{1}$ Department for Internal Medicine 3 and Institute for Clinical \\ Immunology, University of Erlangen-Nürnberg, Erlangen, \\ Germany, ${ }^{2}$ Department for Process Technology and Machinery, \\ University of Erlangen-Nürnberg, Erlangen, Germany, ${ }^{3}$ Center \\ of Life and Food Science, München, Germany, ${ }^{4}$ Department \\ of Radiation Oncology, University of Erlangen-Nürnberg, Erlangen, \\ Germany
}

Various forms of cancer cause the death of every forth human in Germany. Recently described therapeutic approaches are based on the vaccination of cancer patients with autologous, inactivated tumour cells. The master requirements of cell based tumour vaccines are (I) the complete inactivation, (II) the preservation of their immunogenicity, and (III) the accordance with statutory provisions. Currently, chemotherapeutics and physical treatments are used to inactivate tumour cells, but all procedures have methodological, therapeutical, or legal restrictions. Therefore, we employed high hydrostatic pressure $(P>50 \mathrm{MPa})$ for inactivation of tumour cells. 
The treatment of tumour cells with pressure up to $100 \mathrm{MPa}$ decelerated proliferation but did not affect cells' viability. Pressure above $200 \mathrm{MPa}$, however, resulted in cell death without survivers even in long-term culture. Cells pressurized with $200 \mathrm{MPa}$, died in consecutive cultures by apoptosis, whereas pressure above $300 \mathrm{MPa}$ necrotizes the cells during treatment. Most importantly, both pressurized apoptotic and necrotic cells retained their immunogenicity. In contrast to alternative methods for the induction of necrosis (heat, detergent, oxidative stress), the nuclei of HHP treated cells were efficiently degraded by a $\mathrm{Ca}^{2+}$-dependent DNase within a few hours in culture. Furthermore, the viscosity of the cytoplasm increased and soluble cellular constituents are retained within the necrotic cell. In addition, proteinaceous, gelificated cytoplasm conserves the shape of the treated cells for weeks. These features favour HHP as a powerful technique for inactivation of tumour cells, preserving their immunogenicity.

\section{P020}

\section{Reciprocal regulation of the therapeutic effectiveness of cancer vaccines by effector versus memory $\mathrm{CD8}^{+} \mathrm{T}$ cells}

Y. Nakamura, P. Watchmaker, J. Urban, R. Mailliard, P. Kalinski; University of Pittsburgh Cancer Institute, Pittsburgh, PA, USA

$\mathrm{CD}^{+} \mathrm{T}$ cell can activate dendritic cells (DCs) and enhance their Th1-driving and CTL-inducing functions. They can also kill DCs, terminating immune responses. Here, we report that these opposite "helper" versus "suppressor" functions are differentially expressed at different stages of $\mathrm{CD}^{+} \mathrm{T}$ cell activation, being selectively performed by memory versus effector cells. The interaction of DCs with memory $\mathrm{CD}^{+} \mathrm{T}$ cells results with $\mathrm{DC}$ activation, enhancement of their IL-12-producing function, and increased effectiveness in inducing tumor-specific Th1 and CTL responses. In contrast, effector cells kill DCs, terminating their immunostimulatory functions. Using human in vitro and mouse in vivo models, we have observed that promoting DC interaction with these different subsets of $\mathrm{CD} 8^{+} \mathrm{T}$ cells can selectively enhance or suppress the induction of tumor-specific immunity, promoting or eliminating the therapeutic effects of vaccination. The selective performance of helper functions by memory, but not effector $\mathrm{CD}^{+} \mathrm{T}$ cells, indicates the existence of a novel mechanism of immune memory. Our data help to explain poor efficacy of therapeutic vaccines compared to preventive ones and the importance of tumor-specific memory cells for anti-tumor effects, helping to design effective immunotherapeutic strategies against cancer.

\section{P021 \\ Dendritic cells regulate tumor-homing potential and tumoricidal activity of tumor-specific $T$ cells}

\author{
P. Watchmaker, R. Muthuswamy, T. Reinhart, J. Kirkwood, \\ D. Bartlett, P. Kalinski; \\ University of Pittsburgh Cancer Institute, Pittsburgh, PA, USA
}

Dendritic cell-based vaccines have been shown to effectively induce high numbers of circulating tumor-specific T cells, but their ability to induce clinical responses remains limited. In accordance with these data, we observed that the frequently-used nonpolarized "standard" DCs (sDC; induced by the combination of
IL-1 $\beta / \mathrm{TNF} \alpha / \mathrm{IL}-6 / \mathrm{PGE}_{2}$ ), induce substantial expansion of $\mathrm{T}$ cells, but are poorly effective in inducing $\mathrm{T}$ cell effector functions and tumor-relevant homing properties. In contrast, while type-1 polarized DCs $(\alpha \mathrm{DC} 1 \mathrm{~s}$; induced by the combination of IL- $1 \beta /$ $\mathrm{TNF} \alpha / \mathrm{IFN} \alpha / \mathrm{IFN} \gamma / \mathrm{p}-\mathrm{I}: \mathrm{C})$, induce comparable $\mathrm{T}$ cell proliferation, they induce pronounced CTL activity in polyclonal and melanoma-specific $\mathrm{CD} 8^{+} \mathrm{T}$ cells, in addition to their long-term survival. Moreover, $\alpha \mathrm{DCl}$ are superior in inducing $\mathrm{T}$ cell expression of CXCR3 and CCR5, the chemokine receptors mediating $\mathrm{T}$ cell entry into tumors, and associated with prolonged survival of patients with advanced melanoma and with improved response to immunotherapy. We have recently started clinical comparison of the effectiveness of semi-continuous intralymphatic $\alpha \mathrm{DC} 1$ - and sDC-based vaccines against melanoma, and are implementing a trial in the setting of colorectal cancer to determine the preferred route of administration of $\alpha \mathrm{DCl}$-based vaccines.

P022

mRNA transfection of DCs in immature and mature state: comparable in vitro priming of Th and CTL against DCs electroporated with tumor cell line derived mRNA

\author{
S. Jarnjak-Jankovic, S. Saeboe Larssen, G. Kvalheim, \\ G. Gaudernack; \\ The Norwegian RadiumHospital, Oslo, Norway
}

The use of mRNA in vaccine studies has generally been through loading or transfection of immature DC, followed by a maturation step. A recent study has suggested that this strategy may result in inferior CTL priming. Furthermore the study did not address any possible effects on the priming of $\mathrm{CD} 4+\mathrm{T}$ cell response. In this study, we compare mRNA transfection of mature DCs with that of immature DC and use as readout their capacity to prime autologous $\mathrm{T}$ cells during one cycle of in vitro stimulation. In this model system we used mRNA from the tumor cell line Jurkat E6. Our results demonstrate that no differences in the phenotype of DCs transfected either immature stage (day 5) or in the mature stage (day 7) were observed. Interestingly, no major differences in their ability to prime CD4 and CD8 T cell responses were observed. Since in vitro priming to some extent may reflect the capacity of these DCs to prime T cells in vivo after vaccination, these studies support the use of RNA-transfected mature DC in clinical protocols. Transfection of the DCs at the end of the maturation process represents a logistical improvement in the GMP production of mRNA transfected DCs for clinical protocols.

\section{P023}

\section{Cyclin-D1 as a widely expressed tumor antigen: generation of high avidity CTL in an autologous setting}

\author{
E. Kondo ${ }^{1,2}$, M. Weirauch ${ }^{1,2}$, C. Wickenhauser ${ }^{3}, J$. L. Schultze $e^{2}$, \\ M. von Bergwelt-Baildon ${ }^{1,2}$; \\ ${ }^{1}$ Max-Eder Young Investigator Group, University of Cologne, \\ Cologne, Germany, ${ }^{2}$ Molecular Tumor Biology and Tumor \\ Immunology, 1st Department of Internal Medicine, University of \\ Cologne, Cologne, Germany, ${ }^{3}$ Institute of Pathology, University of \\ Cologne, Cologne, Germany
}


Cyclin-D1, a key cell cycle regulator, is over-expressed in multiple types of cancer. Such tumor-associated genes may be useful targets for cancer immunotherapy. We previously demonstrated that antigen-specific CTL can be expanded using CD40-activated B-cells (CD40-Bs) as APC. CD40-Bs efficiently present antigen and prime naïve T-cells in vitro. Using CD40-Bs as sole APC we have developed a highly efficient $T$ cell expansion system that has been used to identify novel epitopes in viral, tumor and autoimmune disorders. While it had previously been suggested that CTL recognizing Cyclin-D1 derived epitopes are eliminated from the repertoire due to thymic deletion, $\mathrm{CD} 8^{+}$T-cells specific for several Cyclin-D1 derived epitopes were readily expandable from HLA-A $* 0201^{+}$normal donors and patients with Cyclin-D1 ${ }^{+}$ cancer in the above system. Such T-cells recognized and efficiently lysed HLA-A*0201 ${ }^{+}$Cyclin-D1 ${ }^{+}$tumor cell lines, indicating that these epitopes are processed and presented. We next cloned the CTL lines by limiting dilution and obtained multiple CTL clones with different avidity. Among these CTL clone 2-9 (half maximal lysis at $5.8 \mathrm{nM}$ ) recognized primary tumor cells from mantle cell lymphoma and plasma cell leukemia patients in an HLA-A*0201restricted manner. Taken together, these data indicate that indeed high-avidity Cyclin-D1 specific CTL are present in the repertoire underlining its potential as a target for cancer immunotherapy.

\section{P024}

\section{Melanoma-associated chondroitin sulfate}

\section{proteoglycan (MCSP): a promising target antigen for cancer immunotherapy?}

\section{Erfurt ${ }^{1}$, I. Haendle ${ }^{1}$, B. Schuler-Thurner ${ }^{1}$, K. Thielemans ${ }^{2}$, P. van der Bruggen ${ }^{3}$, G. Schuler ${ }^{1}$, E. S. Schultz ${ }^{4}$; \\ ${ }^{I}$ Department of Dermatology, University Hospital Erlangen, Erlangen, Germany, ${ }^{2}$ Laboratory of Physiology, Free University of Brussels, Brussels, Belgium, ${ }^{3}$ Ludwig Institute for Cancer Research, Brussels, Belgium, ${ }^{4}$ Department of Dermatology, Marburg, Germany}

To avoid immune escape by downregulation or loss of antigen by the tumor cells target antigens are needed which are important for the malignant phenotype and survival of the tumor. We could identify a CD4 $+\mathrm{T}$ cell epitope derived from the human melanoma-associated chondroitin sulfate proteoglycan (MCSP) (also known as high molecular weight-melanoma-associated antigen $=$ HMW-MAA), which is strongly expressed on $>90 \%$ of human melanoma lesions and is important for the motility and invasion of melanoma cells. However, MCSP is not strictly tumorspecific as it is also expressed in a variety of normal tissues. Therefore, self tolerance should prevent the induction of strong $\mathrm{T}$ cell responses against these antigens by vaccination strategies. On the other hand, breaking self tolerance to this antigen by effectively manipulating the immune system, might mediate antitumor responses although it would bear the risk of autoimmunity. Surprisingly, we could readily isolate CD4 $+\mathrm{T}$ helper cells from the blood of a healthy donor recognizing peptide MCSP693-709 on HLA-DR 11 expressing melanoma cells. Broad T cell reactivity against this antigen could be detected in the peripheral blood of both, healthy donors and melanoma patients, without any apparent signs of autoimmune disease. In some patients, a decline of MCSP-specific $\mathrm{T}$ cell reactivity was observed upon tumor progression. Our data indicate that $\mathrm{CD} 4+\mathrm{T}$ cells are capable of recognizing a membrane glycoprotein that is important in melanoma cell function, and it may be possible that the sizable reactivity to this antigen in most normal individuals contributes to immune surveillance against cancer.

\section{P025}

\section{Genetically modified bone-marrow-derived dendritic cells induce HPV16 E7-specific CTL response in mice}

\author{
K. Dell, L. Gissmann; \\ German Cancer Research Center, Heidelberg, Germany
}

Background: Sexually transmitted high risk human papillomaviruses (HPV) cause cervical cancer. DC-based therapy is a suitable means for the eradication of cervical lesions through the induction of specific cytotoxic T lymphocyte (CTLs) responses.

Methods: CpG-matured DCs were electroprated either with HPV16 E7 plasmids or mRNAs: (i) codon-optimized E7 (E7) or (ii) a shuffled version where the transformation-associated $\mathrm{pRB}$ binding site is disrupted (E7SH). EGFP was used to monitor the effect of transfection on activation marker expression and migration behaviour in vitro. For E7, transfection efficiencies and antigen presentation were determined. The in vivo priming capacity of transfected DCs was evaluated by subcutaneous immunization and evaluation of the E7-specific CTL frequencies.

Results: DC transfection with EGFP-mRNA but not with -DNA enhanced the expression of activation markers and MHC molecules about three-fold. In chemotaxis assays, selectively EGFPmRNA transfected DCs migrated whereas after EGFP-DNA transfection both non-transfected and transfected DCs migrated. All E7 transfected DCs displayed E7 epitopes. The percentage of E7-positive cells was about two-fold higher in E7-DNA and mRNA transfected DCs when compared to the E7SH transfections. The level of E7 expression was strikingly higher in DNA than in mRNA-transfected DCs. Highest E7-specific CTL frequencies were found in mice immunized with E7-DNA transfected DCs followed by E7- and E7SH-mRNA electroporated DCs. E7SH-DNA transfected DCs induced no E7-specific CTL response.

Conclusions: Genetically modified BMDCs are suitable vehicles for the induction of E7-specific CTL frequencies in mice. The most critical aspect for CTL induction seems to be the epitope density.

\section{P026 \\ Induction of high avidity cytotoxic $T$ cells employing dendritic cells transfected with messenger RNA encoding interleukin-12 and tumor-associated antigens}

H. J. Bontkes ${ }^{1}$, D. Kramer ${ }^{1}$, J. J. Ruizendaal ${ }^{1}$, E. W. M. Kueter
,
V. F. I. van Tendeloo ${ }^{2}$, C. J. L. M. Meijer ${ }^{1}$, E. Hooijberg ${ }^{1}$;
${ }^{1}$ VUMC, Amsterdam, The Netherlands, ${ }^{2}$ AUH, Antwerp, Belgium

Dendritic cells (DC) transfected with messenger RNA (mRNA) encoding tumor-associated antigens (TAA) are able to induce potent tumor-specific T-cell responses directed to a broad spectrum of epitopes. The in vitro generation of DC possessing all the features crucial for the induction of type 1 immune responses, such as mature state, migratory potential and interleukin-12 (IL-12p70) production is complicated. Particularly migratory potential is inversely correlated with IL-12p70 production after maturation with prostaglandin E2 (PGE2), which is included in maturation cocktails currently used in most vaccination trials. Here, we show that transfection of PGE2 matured DC with a single mRNA strain encoding for ubiquitin followed by a TAA linked to IL-12 by a self-cleaving $2 \mathrm{~A}$ sequence, 
produced biological active IL-12p70 and were able to present the transfected TAA up to $72 \mathrm{~h}$ after transfection. Furthermore, use of the anti-reverse cap analog for in vitro transcription of the IL12 mRNA enabled constitutive IL- $12 \mathrm{p} 70$ production for up to 5 days. These transfected mature DC migrated efficiently towards lymph node derived chemokines. DCs constitutively expressing IL-12p70, generate TAA-specific cytotoxic T cells with an high functional avidity, independent of CD4 + T-cell help. These results have recently been published by us (H. J. Bontkes et al. in Gene Therapy 14:366-375, 2007). Apoptosis is one of the hallmarks of cancer. Survivin is an inhibitor of apoptosis. It is indispensable for continued tumour growth and is expressed in many different cancers. Here we report on the induction of HLA/A1 restricted survivin specific human $\mathrm{T}$ cells employing the methods described above.

\section{P027}

\section{Profuse and sustained production of MIG/CXCL9} by a clinical grade dendritic cell vaccine: a feature to provide NK cell-dependent boosting for TH1 polarization

K. Gustafsson ${ }^{1}$, M. Persson ${ }^{2}$, L. Bergqvist ${ }^{3}, J$. Alder $^{3}, J$. Nyström $^{2}$, B. Andersson ${ }^{3}$, L. Pellettieri ${ }^{1}$, V. d́Angelo ${ }^{4}$, P. Eriksson ${ }^{1}$,

A. Karlsson-Parra ${ }^{3}$.

${ }^{1}$ Department of Neuroscience and Physiology, Gothenburg, Sweden, ${ }^{2}$ Department of Medicine, Gothenburg, Sweden, ${ }^{3}$ Department of Clinical Immunology, Gothenburg, Sweden, ${ }^{4}$ Department of Neurosurgery, San Giovanni Rotondo, Italy

CXCR3-dependent recruitment of circulating NK-cells into inflamed nymph nodes is known to provide a potent, interferon (IFN)-gamma-dependent, boost for TH1-polarized immune responses in mouse models. Such NK cell recruitment into draining lymph nodes is induced by certain subcutaneously injected adjuvants, including mature dendritic cells (DCs). We here demonstrate that monocyte-derived immature human DCs stimulated with poly I:C in combination with IFN-alpha, IFNgamma, IL-1 beta and TNF-alpha (alpha-DC1) exhibit a sustained and profuse production $\left(>150 \mathrm{ng} / \mathrm{ml} / 10^{6} \mathrm{DCs}\right)$ of the CXCR3-ligand monokine induced by IFNgamma (MIG)/ CXCL9 after withdrawal of maturation stimuli. In contrast, no measurable or low levels $\left(<1 \mathrm{ng} / \mathrm{ml} / 10^{6} \mathrm{DCs}\right)$ of MIG/CXCL9 are produced by DCs after maturation with the current "gold standard" maturation protocol for human DC-based cancer vaccines consisting of TNF-alpha, IL-1 beta, IL-6 and prostaglandin E2 (PGE2-DC). Functional studies in vitro further demonstrate that alpha-DC1-supernatants actively recruit NK cells, and that addition of anti-MIG/CXCL9 antibodies to the supernatant blocks this recruitment. Finally, alpha-DC1, but not PGE2-DC, significantly activate cocultured autologous NK cells as determined by CD69 expression and intracellular IFNgamma production. These novel findings indicate that fully matured and subsequently injected human alpha-DC1-based clinical grade vaccines have the potential to recruit and activate NK-cells during their arrival to draining lymph nodes and that this feature may be of relevance for efficient priming of tumorspecific Th1 cells and CTLs.
P028

Allogeneic monocyte-derived cells loaded with tumor antigens as a combined antigen-delivery vehicle and adjuvant in cancer immunotherapy

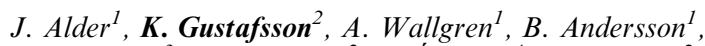
E. Jennische ${ }^{3}$, L. Pellettieri ${ }^{2}$, V. d'Angelo ${ }^{4}$, P. Eriksson ${ }^{2}, S$. Lange $^{5}$, A. Karlsson-Parra ${ }^{I}$;

${ }^{1}$ Department of Clinical Immunology, Gothenburg, Sweden,

${ }^{2}$ Department of Neuroscience and Physiology, Gothenburg, Sweden, ${ }^{3}$ Department of Anatomy and Cell Biology, Gothenburg, Sweden,

${ }^{4}$ Department of Neurosurgery, San Giovanni Rotondo, Italy,

${ }^{5}$ Department of Clinical Bacteriology, Gothenburg, Sweden

We have recently shown that addition of antigen-presenting cells (APCs) into an allogeneic immune compartment in vitro elicits an inflammatory reaction that promotes maturation of bystander dendritic cells with $\mathrm{T}$ helper 1 (TH1)-inducing capacity. We therefore proposed that vaccination with allogeneic, tumor-antigen-loaded, APCs would lead to efficient crosspriming of tumor-specific $\mathrm{T}$ cells. Fisher 344 female rats were challenged subcutaneously with the highly malignant breast cancer cell line 13762MAT B III. The rats were vaccinated in both prophylactic and therapeutic settings. In initial experiments, the vaccine cells consisted of allogeneic peripheral blood monocytes cultivated for 2 days in GM-CSF. After 1 day the monocytes were loaded with apoptotic tumor cells with or without addition of Vibrio cholerae neuraminidase (NAS), an enzyme known to induce inflammatory chemokine production in monocytes and to enhance their allogenicity. Prophylactic vaccinations reduced tumor take from $80 \%$ in non-vaccinated rats to $20 \%$ in vaccinated rats. This immunity was long-lasting since re-challenge of tumor-rejecting rats with tumor cells 6 weeks later failed to induce any tumor growth. In the therapeutic setting all rats developed tumors but tumor growth was significantly reduced in rats given allogeneic and NAS-treated tumor cell loaded monocytes $(P<0.05)$ or monocyte-derived dendritic cells (differentiated with GM-CSF + IL-4 for 5 days, $P<0.001$ ). Our results indicate that NAS-treated allogeneic monocyte-derived cells may act as antigen carrier as well as adjuvant in cancer vaccination and immunotherapy.

\section{P029}

The TLR8 agonist R848 primes human monocyte-derived dendritic cells for a secondary, CD40-dependent, burst of IL-12p70 production
L. Bergqvist ${ }^{1}$, S. Holmgren ${ }^{1}$, S. Johnson ${ }^{1}$, K. Gustafsson ${ }^{2}$, J. Alder ${ }^{1}$, B. Andersson ${ }^{1}$, L. Pellettieri ${ }^{2}$, V. ḋAngelo ${ }^{3}$, P. Eriksson ${ }^{2}$, A. Karlsson-Parra ${ }^{I}$;
${ }^{1}$ Department of Clinical Immunology, Gothenburg, Sweden,
${ }^{2}$ Department of Neuroscience and Physiology, Gothenburg, Sweden, ${ }^{3}$ Department of Neurosurgery, San Giovanni Rotondo, Italy

Effective induction of antitumor CTL responses requires fully mature DCs that express high levels of costimulatory molecules. In addition, high interleukin-12p70 (IL-12p70) secretion dramatically enhances the ability of DC-based vaccines to induce 
tumor-specific Th1 cells and CTLs, and promotes tumor rejection in therapeutic mouse models. A major concern, however, is whether mature DCs are still capable of secreting IL-12p70 after in vivo administration at the time of interaction with CD40 ligand expressing $T$ cells. Here, we report that maturation with the TLR8 agonist R848, but not maturation with LPS or poly-I:C, primes monocyte-dervied human DCs (propagated in a clinically relevant serum-free medium supplemented with GM-CSF and IL-4) for substantial production of IL-12p70 upon CD40 cross-linking 48-72 h apart from initial stimulation. Combining R848 with LPS or poly-I:C induced a significant primary burst of IL-12p70 production followed by strong secondary burst of IL-12p70 production after CD40-mediated restimulation. Addition of IFN-gamma to R848 during the maturation step syngergistically enhanced the secondary, CD40mediated, burst of IL-12p70 production. In contrast, re-stimulation of R848/IFN-gamma-primed DCs with TLR ligands (R848, LPS or poly I:C) did not induce any substantial IL-12p70 production. Our data thus suggest that R848 may be one of the most appropriate agents to generate mature DC by retaining the ability of these DCs to secrete bioactive IL-12 upon CD40-stimulation.

\section{P030 \\ Electroloading of mature monocyte-derived dendritic cells with whole tumor cell lysate elicits robust antigen-specific CTL expansion}

\author{
L. A. Wolfraim ${ }^{I}$, A. M. Viley ${ }^{I}$, R. Shivakumar ${ }^{1}, M$. Nieda ${ }^{2}$, \\ K. Yokokawa ${ }^{2}$, L. Liu ${ }^{l}$, M. V. Peshwa ${ }^{l}$; \\ ${ }^{1}$ MaxCyte, Inc., Gaithersburg, MD, USA, ${ }^{2}$ MEDINET Co., Ltd., \\ Shin-Yokohama, Japan
}

Dendritic cell (DC) vaccines represent a promising approach for the treatment of malignancies. However, clinical success has been limited. Greater success may be achieved through the manufacture of more potent DC vaccines. Current approaches involve co-culture of immature DC (imDC) with whole tumor cell lysates. This laborious, non-robust process leads to suboptimal antigen uptake and necessitates subsequent maturation of loaded imDC to generate potent immuno-competent DC. An alternative approach uses electro-loading. This method of antigen loading circumvents the innate antigen capture abilities of the imDC and is theoretically not restricted to the use of imDC alone and can be effectively used with $\mathrm{mDC}$. We have developed a robust, clinical scale, cGMP and regulatory compliant process, utilizing sterile single-use disposable technology for electroloading whole tumor cell lysates into previously matured DC (mDC) in a closed system manufacturing process. Viability and phenotype is essentially identical to DC vaccine generated by conventional lysate co-culture processes. In direct head-to-head comparisons, electroloaded mDC consistently elicited enhanced antigen-specific $\mathrm{T}$ cell expansion compared to DC vaccines generated using traditional co-incubation, while requiring significantly lower amount of tumor lysate. Electroloading shortened the entire manufacturing process by one day, achieved more efficient loading per cell, and used less total lysate than conventional lysate co-culture process. The developed technology uses MaxCyte's proprietary platform technologies, described in a Master File with CBER, United States FDA, is cross referenced in multiple clinical trials and represents an opportunity for economical translation for clinical/ commercial manufacture and delivery of a DC product with enhanced potency.
P031

B subunit of Shiga toxin based vaccines synergize with alpha-GalCer to break tolerance against self antigen and elicit anti-tumor immunity

O. Adotevi ${ }^{1}$, B. Vingert ${ }^{1}$, L. Freyburger ${ }^{2}$, F. Quintin-Colonna ${ }^{2}$, M. Amessou ${ }^{3}$, A. Herbelin ${ }^{4}$, W. H. Fridman ${ }^{5}$, L. Johannes $^{3}$, E. Tartour ${ }^{2}$;

${ }^{1}$ Universite Paris-Descartes (Paris 5), Paris, France, ${ }^{2}$ Universite Paris-Descartes (Paris 5)-ENVA, Paris, France, ${ }^{3}$ Institut Curie, Paris, France, ${ }^{4}$ CNRS FRE 2444 Université Paris Descartes. Hal Necker, Paris, France, ${ }^{5} U M R-S 872$ Centre de recherche des Cordeliers., Paris, France

The non toxic B subunit of Shiga toxin (STxB) targets in vivo antigen to dendritic cells which preferentially express the glycolipid Gb3 receptor. After administration of STxB chemically coupled to ovalbumin (STxB-OVA) or E7, a polypeptide derived from HPV in mice, we showed that the addition of $\alpha$-GalCer resulted in a dramatic improvement of the STxB antigen delivery system as reflected by the more powerful and longer lasting $\mathrm{CD} 8^{+} \mathrm{T}$ cell response observed even at very low dose of immunogen $(50 \mathrm{ng})$. This synergy was not found with other adjuvants (CpG, Poly(I:C), IFN $\alpha$ ) also known to promote dendritic cell maturation. With respect to the possible mechanism explaining this synergy, mice immunized with $\alpha$-GalCer presented in vivo the $\mathrm{OVA}_{257-264} / \mathrm{K}^{\mathrm{b}}$ complex more significantly and for longer period than mice vaccinated with STxB alone or mixed with other adjuvants. To test whether this vaccine could break tolerance against self antigen, ovalbumin transgenic mice were immunized with STxB-OVA alone or mixed with $\alpha$-GalCer. Although no CTL induction was observed after immunization of ovalbumin transgenic mice with STxB-OVA alone, tetramer assay clearly detected specific anti-OVA $\mathrm{CD} 8{ }^{+} \mathrm{T}$ cells in 8 out of 11 mice immunized with STxB-OVA combined with $\alpha$-GalCer. In addition, vaccination with STxB-OVA and $\alpha$-GalCer efficient to cure established tumor expressing Ovalbumin. STxB combined with $\alpha$-GalCer therefore appears as a promising vaccine strategy to more successfully establish protective $\mathrm{CD} 8^{+} \mathrm{T}$ cell memory against tumors.

\section{P032}

Delivery of tumor antigens using a membrane translocating peptide to dendritic cells induces cellular and protective immune responses

\author{
D. S. Pouniotis, V. Apostolopoulos, G. Pietersz; \\ Macfarlane Burnet Institute for Medical Research and Public \\ Health, Heidelberg, Melbourne, Australia
}

Cytoplasmic delivery and cross-presentation of proteins and peptides is necessary for processing and presentation of antigens for the generation of cytotoxic $\mathrm{T}$ cells. We previously described the use of the 16 amino acid peptide penetratin from the Drosophila Antennapedia domain (penetratin, Antp) to transport CTL epitopes into cells. We show that penetratin covalently linked to different proteins facilitates more rapid uptake by dendritic cells (DCs). In addition, penetratin linked in tandem to $\mathrm{CD} 4^{+}$and/or $\mathrm{CD}^{+} \mathrm{T}$ cell epitopes stimulate $\mathrm{T}$ cells in vitro. Penetratin is responsible for mediating uptake of peptides/proteins by DCs and induces superior antigen-specific responses in vivo. The internalization mechanism of penetratin conjugates shows diverse uptake and MHC class I processing mechanisms dependent on the cargo shown with specific biochemical inhibitors and by confocal 
microscopy. The immune responses generated by penetratin immunization can also suppress tumor growth in mice. Tumor protection is mediated by a $\mathrm{CD} 8^{+} \mathrm{T}$ cell dependent mechanism and does not require $\mathrm{CD} 4^{+} \mathrm{T}$ cell help to protect mice 7 days after immunization. Alternatively, 40 days after the last immunization, the presence of $\mathrm{CD} 4^{+} \mathrm{T}$ cell help enhances antigen-specific IFN- $\gamma$ secreting $\mathrm{CD} 8{ }^{+} \mathrm{T}$ cells in vivo and improves tumor protection in mice. In addition, a single immunization of penetratin conjugates can significantly delayed growth of tumors in mice in a tumor therapy model. We are currently investigating the use of penetratin to deliver novel multiple antigen peptides (MAPs) incorporating multiple $\mathrm{CD} 4^{+}$and $\mathrm{CD}^{+} \mathrm{T}$ cell epitopes from tumor antigens to DCs to induce immunity.

\section{P033}

Tumor antigen-loaded mouse dendritic cells maturing in presence of inflammatory cytokines are potent activators of immune response in vitro but not in vivo

\section{J.Rossowska, E. Pajtasz-Piasecka, A.Szyda, A. Krawczenko, D.Duś; Institute of Immunology and Experimental Therapy, Wrocaw, Poland}

The use of tumor antigen-loaded DCs (TAg/DCs) is one of the most promising approaches to induce a tumor-specific immune response. However, methods of the vaccine preparation have not yet been standardized. The purpose of the work was to analyze the antitumor efficacy of TAg/DCs at different stages of maturation. DCs were loaded with MC38 colon carcinoma cell lysate (TAg) alone to become partially differentiated or additionally stimulated with inflammatory cytokines such as TNF-alpha, IFN-gamma or IL-12 to reach maturation stage. It was observed that $\mathrm{TAg} / \mathrm{DCs}$, simultaneously treated with cytokines especially with IL-12 or IFNgamma + IL-12 were more efficient immune response activators in vitro compared with partially differentiated TAg/DCs. However, injected peritumorally resulted only in insignificant tumor growth delay. Unexpectedly, the highest tumor growth inhibition was noticed when mice were treated with partially differentiated TAg/DCs. Additionally, the capacity of DCs transduced with IL-12 gene (DCs/IL-12) to augment immune response activated by TAg/DCs in different maturation stage was examined. Conducted in vitro as well as in vivo experiments showed that optimal anti-tumor effect was reached only when partially mature $\mathrm{TAg} / \mathrm{DCs}$ were used together with DCs/IL-12. It can suggest that DCs do not have to reach fully mature stage during stimulation in vitro to evoke strong antitumor response. Furthermore, simultaneous application of partially differentiated $\mathrm{TAg} / \mathrm{DCs}$ with $\mathrm{DCs} / \mathrm{IL} 12$, which enable to control in vivo further maturation process of $\mathrm{TAg} / \mathrm{DC}$, seems to be of great importance.

This work was supported by the Ministry of Science and Higher Education (grants:PBZ-KBN-091/PO5/2003,2798/P01/ 2007/32).

\section{P034 \\ Comparative studies on anti-tumor effect of IL-2-transduced murine dendritic cell and tumor cell vaccine \\ E. Pajtasz-Piasecka ${ }^{1}, J$. Rossowska ${ }^{1}, J$. Wojas $^{2}$, A. Szyda ${ }^{1}$, D. Dus ${ }^{1}$; \\ ${ }^{I}$ Institute of Immunology and Experimental Therapy, Wroclaw, Poland, ${ }^{2}$ Wroclaw University of Technology, Wroclaw, Poland}

The attempts of immunotherapy with IL-2 as a potent immunomodulator of anti-tumor reactions increase in their number. To overcome the side effects caused by the systemic IL-2 therapy, the cytokine could be replaced by local use of IL-2producing cells applied as adjuvant vaccines enhancing an antitumor immunity.

In presented work murine colon carcinoma (MC38/0) cells or JAWS II dendritic cells were transduced with retroviral murine IL-2 gene (MC38/IL-2, JAWS II/IL-2). The IL-2 activity ranged $100-150 \mathrm{LU} / \mathrm{ml}$ or $200-350 \mathrm{LU} / \mathrm{ml} / 5 \times 10^{5}$ cells $/ 48 \mathrm{~h}$, respectively. MC38/IL-2 cells demonstrated lack of the tumorigenicity in syngeneic mice. MC38/IL-2 and JAWS II/IL-2 cells were used as a vaccine for peritumoral treatment of tumor-bearing $\mathrm{C} 57 \mathrm{BL} / 6$ mice. Administration of transductants delayed the time required to reach tumor volume of $1 \mathrm{~cm}^{3}$ about 6 days compared with control. However, the combination of JAWS II/IL-2 (but not MC38/IL-2) cells with JAWS II cells activated with MC38 cell lysate (JAWS II/TAg cells) resulted in extent of the tumor growth delay. Re-stimulated in vitro splenocytes obtained from mice vaccinated with JAWS II/IL-2 + /- JAWS II/Ag cells revealed increased cytotoxicity towards $\mathrm{MC} 38 / 0$ cells compared with splenocytes from MC38/IL-2 treated mice. Cytotoxic activity was accompanied by increased number of $\mathrm{CD} 8{ }^{+} \mathrm{CD} 107^{+}$cells and $\mathrm{CD}_{49} \mathrm{~b}^{+} \mathrm{CD} 107^{+}$cells and decreased IFN-gamma secretion. The obtained data suggested that the dendritic cells transduced with IL-2 gene used as a vaccine in the anti-tumor therapy could be a more potent carrier of the cytokine than tumor cells.

This work was supported by the Ministry of Scientific and High Education (grants: PBZ-KBN-091/PO5/2003, 2798/P01/2007/32).

\section{P035}

\section{IFN $\alpha$ skews monocytes into CD56 + expressing dendritic cells with potent functional activities in vitro and in vivo}

\author{
C. Papewalis ${ }^{1}$, B. Jacobs ${ }^{1}$, M. Wuttke ${ }^{1}$, M. Cohnen ${ }^{2}$, \\ W. A. Scherbaum ${ }^{l}$, M. Schott ${ }^{1}$; \\ ${ }^{1}$ Departement of Endocrinology, Duesseldorf, Germany, \\ ${ }^{2}$ Departement of Radiology, Duesseldorf, Germany
}

The anti-tumor effect of IFN $\alpha$ is mediated by the activation of CTLs and the generation of dendritic cells (IFN-DCs) from monocytes. The aim of this study was to characterize IFN-DC in more detail and to use them in vivo.

IFN-DCs were generated from human monocytes with GM-CSF and IFN $\alpha$. These cells showed phenotypical characteristics of IFN-DCs as formerly described. Most important, a major part $(55.8 \pm 13.9 \%)$ of these cells expressed CD56, a marker which has been thought to be specific for NK cells. FACS analyses of CD56 + and CD56- subsets showed a similar pattern of classical DC marker. Importantly, CD56 + IFN-DCs exhibit cytolytic activity up to $24 \%( \pm 4 \%)$ which could be blocked $(-80.6 \pm 1.8 \%)$ by culturing with anti-TRAIL. Cytokine analyses of the supernatent of CD56 + IFN-DC showed a large increase of IFN $\gamma(352.5 \mathrm{pg} / \mathrm{ml} \pm 2.2 \%)$ after coculture with K562 tumor cells. Mixed leucocyte reaction revealed a stronger capacity in stimulating allogenic T cells of CD56 + compared to CD56- IFNDC. Five patients with metastasized medullary thyroid carcinoma were treated with IFN-DCs resulting in two stable diseases (follow-up 37 months). Immunohistochemical analyses of DTH skin reaction showed a strong infiltration of CD8 + CTLs. Intracytoplasmatic cytokine analyses revealed an increase of calcitoninspecific IFN $\gamma$-secreting CD4 + cells in peripheral blood in treated patients. 
In summary, we here describe that cytotoxic activity of IFN-DCs is mainly mediated by a subset of IFN-DCs showing partial phenotypic and functional characteristics of NK cells. These cells represent one mechanism of the anti-tumor effect induced by IFN $\alpha$.

\section{P036}

\section{Idiotype-KLH loaded dendritic cell vaccination in patients with stage I multiple myeloma-evaluation of immune responses by different read-out systems}

\author{
S. Auffermann-Gretzinger ${ }^{1}$, C. Schmidt ${ }^{1}$, M. Schmitz ${ }^{2}$, \\ G. Ehninger ${ }^{1}, M$. Bornhäuser ${ }^{1}$; \\ ${ }^{I}$ Med. Klinik und Poliklinik I, Universitätsklinik Dresden, Dresden, \\ Germany, ${ }^{2}$ Institut für Immunologie, Universitätsklinik Dresden, \\ Dresden, Germany
}

Immunotherapy of cancer with DC vaccines has produced encouraging results in clinical trials. Antigen-pulsed DC have elicited CD4 + and CD8 + T-cell immunity and tumor regression in humans. In multiple myeloma vaccination has been studied mostly after chemotherapy and induction of a clinical response. Here we investigated anti-idiotypic and $\mathrm{KLH}$ immune responses in stage I multiple myeloma patients vaccinated with monocyte derived dendritic cells pulsed with idiotypic protein conjugated to KLH. Patients $(n=10)$ were vaccinated either s.c. or i.v. five times in 4-week intervals. Idiotype-specific T-cell responses were assessed by $\left[{ }^{3} \mathrm{H}\right]$ thymidine incorporation, cytokine bead assay $\left(\mathrm{TH}_{1}\right.$ IFN- $\gamma, \mathrm{TNF}-\alpha$, IL-2/TH 2 IL-4, IL-5, IL-10), and clinical immune status. In $9 / 10$ patients an Idiotype-specific proliferative immune response could be detected, in $6 / 9$ this was sustained. $7 / 10$ patients developed an idiotype specific $\mathrm{TH}_{1}(4 / 10), \mathrm{TH}_{2}(5 / 10)$ Type cytokine secretion. Over the course of vaccination $6 / 10$ patients developed increased numbers of blood of CD4 T cells, 4/10 showed increased CD8 T cells. 6/10 had more circulating B cells, in $4 / 10$ patients the number of CD56 NK cells increased during the vaccination. All patients developed a strong and sustained proliferation and cytokine secretion in response to KLH. Taken together, these results indicate that idiotype immunization of stage I myeloma patients may induce specific T-cell responses and application of multiple read-out systems improves the characterization of the immune response. In addition, further vaccine development is necessary to increase the proportion of patients that make sustained and therapeutically efficient Idiotype-specific immune responses.

\section{P037}

CD4 and CD8 $\mathrm{T}$ cell responses to $\mathrm{p33}^{\mathrm{ING1b}}$ and p9 $^{\text {ING4 }}$ tumor associated antigens as new immune targeted strategy in patients with renal cell carcinoma

\section{E. Nichiporuk, M. Gasser, M. Grimm, F. Hillig, B. Kneitz, H. Riedmiller, A. Waaga-Gasser; \\ University of Wuerzburg, Wuerzburg, Germany}

The inhibitor of growth (ING) genes play an important role in cell cell cycle arrest and apoptosis through modulating p53 tumor suppressor gene. Recent data have shown that defective function of these genes promotes further tumor growth and progression.
As ING isoforms like $\mathrm{p} 33^{\mathrm{ING} 1 \mathrm{~b}}$ are overexpressed intracellularly and on the cell surface of certain tumors they may be important tumor associated antigens for effective tumor immune responses. In renal cell carcinoma expression and function of ING-isoforms are unknown. Here we analyzed ING gene expression and T-cell mediated immune responses in terms of specific $\mathrm{p} 33^{\mathrm{ING} 1 \mathrm{~b}}$ and p $29^{\text {ING4 }}$ peptide sequences as well as T-regulatory cells. We analyzed tumor tissues of 41 patients with respect to specifically overexpressed ING-isoforms and studied T-cell subsets in vitro obtained from peripheral blood lymphocytes with their relevant cytokine expression. In addition, we assessed the role of different ING peptide sequences for the initiation of tumor immune responses in these patients. In this study we demonstrate for the first time that $\mathrm{p} 33^{\mathrm{ING} 1 \mathrm{~b}}$ and $\mathrm{p} 29^{\mathrm{ING} 4}$ are significantly upregulated in patients with renal cell carcinoma triggering tumor specific immune responses in these patients. Particularly the epitope p $29^{\mathrm{ING} 4}$ aa 149-158 elicited in vitro a significant IFN- $\gamma$ response, thus, enhancing anti-tumor directed immune responses. In conclusion our results suggest an effective immune modulating effect of ING proteins in patients with renal cell carcinoma. Thus they may play an important role as tumor associated antigens which make them attractive targets for a T-cell mediated immunotherapy.

\section{P038}

\section{CCL19 (ELC) as an adjuvant for DNA vaccination}

\author{
G. Baldenhofer ${ }^{1,2}$, T. Nguyen-Hoai ${ }^{1,2}$, U. E.Höpken ${ }^{2}$, M. Lipp ${ }^{2}$ \\ B. Dörken ${ }^{1,2}$, A. Pezzutto ${ }^{1,2}$, J. Westermann ${ }^{1,2}$. \\ ${ }^{1}$ Charité-Campus Berlin-Buch, Berlin, Germany, ${ }^{2}$ Max Delbrück \\ Center for Molecular Medicine, Berlin, Germany
}

Genetic immunization using naked DNA is an attractive approach for immunotherapy. However, clinical application is hampered by a low immunogenicity of DNA vaccines in humans. Therefore, coexpression of tumor antigens together with immunomodulatory molecules is used as a strategy in DNA vaccination aiming at an amplification of the antitumor immune response. Ebstein-BarrVirus-induced-molecule-1-ligand-chemokine (ELC/CCL19) is a $\mathrm{CC}$ chemokine which binds to the chemokine receptor CCR7 on dendritic cells (DC) and particular subsets of T cells.

We used plasmid DNA (pDNA) coding for CCL19 (ELC) as an adjuvant for DNA vaccination in two different mouse tumor models with b-galactosidase (b-gal) or Her2/neu as target antigens.

Mice were vaccinated twice intramusculary on days 1 and 15 . Tumor challenge with b-gal- or Her2/neu-positive syngeneic tumor cells was performed subcutaneously on day 25 . Coadministration of pDNA(tumor antigen) plus pDNA(CCL19) was compared with pDNA(tumor antigen), pDNA(CCL19), mock vector and PBS alone. Coexpression of CCL19 resulted in enhancement of a TH1-polarized immune response with substantial improvement of the protective effect of the DNA vaccine. Immunohistochemical staining revealed an increased CD8 $+\mathrm{T}$ cell infiltration in the tumor tissue of mice which had been immunized with pDNA(tumor antigen) plus pDNA(CCL19). We conclude that CCL19 is an attractive adjuvant for DNA vaccination, able to augment antitumor immunity and that this effect is partially caused by enhanced CD8 $+\mathrm{T}$ cell recruitment. DNA vaccination with Her2/neu plus CCL19 might be a promising approach in breast cancer, particulary in the clinical situation of minimal residual disease after surgery and/or systemic therapy. 


\section{TLR-Stimulation}

\section{P039}

\section{Interleukin-10 severely impairs TLR mediated activation of antigen presenting cells}

\author{
A. Knödler, A. Bringmann, M. M. Weck, K. M. Brauer, \\ L. Kanz, F. Grünebach, P. Brossart; \\ Abteilung für Hämatologie, Onkologie, Immunologie, Rheumatol- \\ ogie und Pulmologie, Universitätsklinikum Tübingen, Tübingen, \\ Germany
}

Activation and the successive phenotypic and functional changes of dendritic cells (DC) via Toll-like receptors (TLR), is believed to be a critical event regulating the strength and outcome of an immunological response. IL-10 is an immunosuppressive cytokine shown to have inhibitory effects on function and differentiation of APC by interfering with signaling pathways involved in DC activation like PI3 kinase or NFkB.

In our study we analyzed the function of human DCs generated in the presence of IL-10 upon activation by Toll-like receptor ligands (TLR2, TLR3, TLR4, TLR7/8). Exposure of DCs to IL-10 resulted in a skewed phenotypic maturation in response to stimuli provided by TLR ligands, a reduced cytokine production like IL-12, IL-6 or TNF-alpha and impaired capacity to stimulate $\mathrm{T}$ cell activation. Furthermore, CCR7 upregulation in DC exposed to TLR stimulation as well as migration toward CCL19/ MIP-3beta were strongly reduced. As a suitable mechanism for these effects, IL-10 was found to down regulate MyD88, an essential adaptor molecule for TLR signaling, and to decrease TLR-induced nuclear expression of the NF-kappaB transcription factor members c-Rel and Rel-B as well as IRF-3 and IRF-8. This was not due to the inhibition of the MAP kinase pathway as phosphorylation of p38 and ERK was not affected but was rather mediated by inhibitory effects on the PI3 kinase pathway. IL-10 treatment of APC resulted in a reduced phosphorylation of TLR induced mTOR phosphorylation. In summary, our results demonstrate that IL-10 can inhibit TLR-mediated activation of APC via a MyD88-dependent and independent pathway.

\section{P040 \\ DC vaccination efficacy is dependent on the duration of DC activation by CpG oligonucleotides in the $\mathrm{C26}$ colon carcinoma model}

\section{Wurzenberger, S. Heidegger, S. Schreiber, S. Weigel, $V$. Koelzer, S. Endres, C. Bourquin, \\ Clinical Pharmacology, Munich, Germany}

CpG motif-containing oligonucleotides (CpG), ligands for TLR9, are potent activators of murine DC. We have recently shown that $\mathrm{CpG}$ in combination with antigen-pulsed, $\mathrm{CpG}$-matured DC can elicit a strong CD8 T cell response, leading to rejection of tumors in the $\mathrm{C} 26$ mouse model of colon carcinoma. Here we show that the kinetics of $\mathrm{DC}$ activation with $\mathrm{CpG}$ oligonucleotides are critical for the outcome of tumor immunotherapy based on antigen-pulsed DC in the C26 mouse model of colon cancer.

Upon in vitro stimulation with $\mathrm{CpG}$, bone marrow-derived DC upregulated expression of costimulatory molecules and produced inflammatory cytokines including IL-12p70. Cytokine secretion was detectable as early as $4 \mathrm{~h}$ after stimulation and persisted after removal of the stimulus. Maximum cytokine production was reached in DC activated for $4 \mathrm{~h}(4 \mathrm{~h}-\mathrm{DC})$ and was not increased by a longer activation of up to $48 \mathrm{~h}$.

Although $4 \mathrm{~h}$-DC appeared fully activated, they did not induce an effective antitumor response. While antigen-pulsed $20 \mathrm{~h}$-DC induced a potent immune response resulting in rejection of established C26 tumors, 4 h-DC provoked only a slight reduction in tumor growth. The migratory potential and the capacity to induce $\mathrm{T}$ cell proliferation were similar in $4 \mathrm{~h}-\mathrm{DC}$ and $20 \mathrm{~h}-\mathrm{DC}$, while $20 \mathrm{~h}$-DC showed a significant increase in the expression of co-stimulatory molecules.

In conclusion, we show that DC need to be activated for $20 \mathrm{~h}$ to reject established tumors in the $\mathrm{C} 26$ model. We suggest that the duration of DC activation is critical for the outcome of immunotherapy in cancer patients.

\section{P041}

\section{CpG oligonucleotides counteract 5-fluorouracil- mediated adverse effects on myeloid cells}

\author{
S. Schreiber ${ }^{1}$, J. Diebold ${ }^{2}$, C. Wurzenberger ${ }^{1}$, N. Sandholzer ${ }^{I}$, \\ S. Endres ${ }^{1}$, C. Bourquin ${ }^{1}$; \\ ${ }^{1}$ Division of Clinical Pharmacology, Department of Internal \\ Medicine, Ludwig Maximilian University, Munich, Germany, \\ ${ }^{2}$ Institute of Pathology, Kantonsspital Luzern, Lucerne, Switzerland
}

$\mathrm{CpG}$ oligonucleotides activate innate immunity by binding to Toll-like receptor 9. Ongoing clinical trials with $\mathrm{CpG}$ oligonucleotides exploit their immunostimulatory potential as vaccine adjuvant and for antitumoral immunotherapy. We previously demonstrated that the antitumoral efficacy of an immunotherapy protocol based on the combination of $\mathrm{CpG}$-activated, mature bone marrow-derived dendritic cells with $\mathrm{CpG}$ oligonucleotides is not affected by simultaneous chemotherapy in a murine colon carcinoma model. Furthermore, while therapeutic doses of 5-fluorouracil alone were associated with dose-limiting toxicity in this model, simultaneous application of dendritic cell vaccination and $\mathrm{CpG}$ oligonucleotides completely abolished chemotherapy-associated toxicity. Here we characterized the protective effect of immunotherapy on 5-fluorouracil toxicity. In non-tumor-bearing mice, $\mathrm{CpG}$ oligonucleotides improved survival to high-dose 5-fluorouracil even in the absence of dendritic cell vaccination. The major adverse effect associated with bolus application of 5-fluorouracil is myelotoxicity with severe neutropenia, lymphopenia and disrupted bone marrow architecture. Whereas 5-fluorouracil-induced neutropenia could not be counteracted by $\mathrm{CpG}$ oligonucleotides, $\mathrm{CpG}$ application enhanced cellularity in the bone marrow. This was especially the case for cells of myeloid lineage. In the spleen, both myeloid and lymphoid subsets were slightly increased after simultaneous application of $\mathrm{CpG}$ oligonucleotides compared to treatment with chemotherapy alone. We hypothesize that $\mathrm{CpG}$ oligonucleotides mediate this survival benefit by accelerating regeneration of the myeloid compartment after 5-fluorouracil-associated toxicity. Enhancement of function and activity of innate effector cells may also be involved. Such effects may have clinical potential by supporting recovery from myelotoxic chemotherapy in tumor patients. 


\section{P042}

\section{Tumor immunotherapy with CpG oligonucleotides: impact on tumor-infiltrating dendritic cells and lymphocytes}

\author{
M. Golic, V. Kölzer, C. Wurzenberger, S. Endres, \\ D. Anz, C. Bourquin; \\ Divison of Clinical Pharmacology, Department of Internal \\ Medicine, Ludwig-Maximilian University of Munich, 80336 \\ Munich, Germany
}

Oligonucleotides containing $\mathrm{CpG}$ motifs (CpG ODN) induce a Th1-mediated immune response and proinflammatory cytokine production via the Toll-like receptor 9 expressed on plasmacytoid dendritic cells and B cells. Tumor immunotherapy with $\mathrm{CpG}$ ODN leads to tumor regression in many experimental cancer models and is currently under investigation in phase III clinical studies of human cancer. However, a systematic analysis of the role of $\mathrm{CpG}$ ODN in the recruitment of lymphocytes and antigenpresenting cells to the tumor has to date not been described.

We analyzed leukocyte infiltration in subcutaneous C26 tumors of mice treated by $\mathrm{CpG}$ ODN using immunohistochemical methods. We show an accumulation of CD11c-positive dendritic cells within the tumors of $\mathrm{CpG}$-treated mice. These cells are distributed throughout the tumor with regional cluster building. While CD3- and CD8-positive cell infiltrates were present in untreated tumors, an increase in the number of infiltrating cells was observed upon $\mathrm{CpG}$ immunotherapy, associated with an increase in perforin-positive cells. Furthermore, we show that CpG ODN administration leads to a decrease in B220-positive cells in tumors.

In summary, we show that CpG ODN-based immunotherapy influences the migration of immune cells to the tumor. Since the immune response against the $\mathrm{C} 26$ colon carcinoma is reported to be CD8-dependent, the increase in cytotoxic effector cells within the tumor upon $\mathrm{CpG}$ immunotherapy may contribute to tumor rejection. Dendritic cells might act as mediators in this process by providing an appropriate milieu for CD8 cell function.

\section{P043}

Toll-like receptor repertoire in tolerogenic dendritic cells and its functional relevance

\author{
S. Chamorro ${ }^{1}$, J. J. Garcia-Vallejo ${ }^{1}$, W. Unger ${ }^{2}$, B. O. Roep ${ }^{2}$, \\ B. A. 't Hart', Y. van Kooyk', \\ ${ }^{1}$ VUmc, Amsterdam, The Netherlands, ${ }^{2}$ LUmc, Leiden, \\ The Netherlands, ${ }^{3}$ BPRC, Rijswijk, The Netherlands
}

Dendritic cells (DC) can sense danger signals that lead to protective responses against pathogens via Toll-like receptors (TLR). Conventional DC (myeloid and plasmacytoid DC) display complementary sets of Toll-like receptors. However, little is known about the TLR repertoire on tolerogenic DC (TDC) and their functional relevance.

We investigated the expression of TLR1 to TLR10 by RT-PCR in different human DC subtypes. We included in our study monocyte-derived DC (mo-DC) and three distinct types of tolerogenic DC (TDC): IL10-treated DC (IL10-DC), dexamethasone-treated DC (DX-DC) and 1,25 (OH $)_{2}$ vitamin D3-treated DC (D3-DC).

Our results show that TDC are endowed with the same TLR set than mo-DC. Interestingly, expression levels of several TLR are significantly upregulated in DX-DC (TLR1, 2, 4 and 5) and
D3-DC (TLR1). Unlike mo-DC, all TDC showed a significant increase in TLR2 upon LPS stimulation. We looked further in the functionality of these cells using agonists for TLR1/2 ( $\left.\mathrm{Pam}_{3} \mathrm{cysk}_{4}\right)$, TLR3 (poli I:C), TLR4 (LPS) and TLR5 (Flagellin) by maturation and cytokine production assays. TDC remained in a semimature state by expressing intermediate levels of costimulatory and class II molecules, with low or no release of pro-inflammatory cytokines and moderate to high levels of anti-inflammatory cytokines. Furthermore TDC exhibited considerably reduced allostimulatory properties as measured in primary mixed lymphocyte reaction.

In conclusion, TDC are able to induce an anti-inflammatory immunosuppressive programme regardless the TLR triggered. From a therapeutic point of view TDC might be beneficial for prevention and/or treatment of autoimmune diseases, allergies and transplantation.

\section{P044}

Assembly and preliminary evaluation of melanoma RNA vaccines coupling MHC-I peptide presentation to TLR signaling

\author{
A. Pato ${ }^{1,2}$, G. Cafri ${ }^{1,3}$, M. Lotem $^{2}$, S. Frankenburg ${ }^{2}$, \\ A. Margalit ${ }^{1,4}$, G. $_{\text {Gross }}{ }^{1,4}$; \\ ${ }^{1}$ MIGAL, Kiryat Shmona, Israel, ${ }^{2}$ Sharett Institute of Oncology, \\ Hadassah University Hospital, Jerusalem, Israel, ${ }^{3}$ Department \\ of of Human Microbiology, Sackler Faculty of Medicine, Tel Aviv \\ University, Tel Aviv, Israel, ${ }^{4}$ Tel-Hai Academic College, Upper \\ Galilee, Israel
}

Priming of CTLs entails their productive encounter with activated dendritic cells (DCs), which display sufficient MHC-I-peptide ligands and provide adequate costimulatory signals. Optimizing CTL priming is a prerequisite for the elicitation of an effective anti-tumor response by DC-based vaccines.

We have recently created a potentially universal genetic vaccine platform, based on membrane-anchored $\beta_{2}$ microglobulin $\left(\beta_{2} \mathrm{~m}\right)$. This design combines MHC-I stabilization with exceptional presentation of pre-selected peptides covalently linked to $\beta_{2} \mathrm{~m}$, and we previously demonstrated its potency in a series of in-vivo experiments in a mouse melanoma model.

In an ongoing study we showed that the incorporation of the signaling domain of toll-like receptor 4 (TLR4) as the anchor portion confers a constitutively activated phenotype on transfected APCs. In the present work we implemented this design in RNA vaccines directed against human melanoma and evaluated their function in ex-vivo propagated human DCs. The HLA-A2-binding gp100 209-217 or MART1 $1_{27-35}$ melanoma peptides were expressed in the context of $\beta_{2} \mathrm{~m}$-human TLR4 or $\beta_{2} \mathrm{~m}-\mathrm{A} 2$ (an HLA-A2-derived anchor). In stable $\mathrm{T} 2$ transfectants the polypeptide product forms functional complexes with HLA-A2. RNA-transfected DCs potently stimulate a CTL line in a peptide specific manner. Transfection of immature DCs with gp100 $209-217-\beta_{2}$ m-TLR4, but not with gp $100_{209-217}-\beta_{2} \mathrm{~m}-\mathrm{A} 2$, considerably upregulates surface expression of CD80, CD83 and CD86. Our findings confirm that the integrated TLR4 signaling domain is functional, while the ability of the extracellular peptide- $\beta_{2} \mathrm{~m}$ portion to pair with MHC-I heavy chains and generate ample CTL ligands is preserved. Comparative evaluation of vaccine efficacy in different in-vivo and ex-vivo settings is underway. 


\section{P045}

\section{Targeting different dendritic cell subsets in the human melanoma sentinel lymph node to boost anti-tumor T cell immunity}

B. G. Molenkamp, B. Sluijter, R. J. C. L. Vuylsteke, S. Meijer,

P. G. J. T. Wijnands, A. J. M. van den Eertwegh,

P. A. M. van Leeuwen, R. J. Scheper, T. D. de Gruijl;

$V U$ medical center, Amsterdam, The Netherlands

Early melanoma development is accompanied by impaired immune effector functions in the tumor-draining sentinel lymph node (SLN). A reduced frequency and activation state of dendritic cells (DC) may interfere with the activation of anti-tumor effector T cells and thus contribute to early metastatic events. Immunopotentiation of the SLN to boost tumor specific immunity may be a valuable adjuvant treatment option that can be generally applied.

We have performed two Phase II studies of Stage-I-III melanoma patients who received either Granulocyte/MacrophageColony Stimulating Factor (GM-CSF) or the CpG-B oligodeoxynucleotide PF-3512676 (formerly CpG 7909), i.d. around the excision site of the primary melanoma. Both resulted in increased frequencies of tumor antigen-specific CD8 $+\mathrm{T}$ cells in the corresponding SLN (determined by IFN $\gamma$ Elispot assay and compared to control groups of patients receiving plain saline). The observed rise in melanoma-reactive CD $8+T$ cells upon GM-CSF administration was associated with an increased frequency of (most likely skin-derived) CD1a $+\mathrm{CD} 83+$ myeloid DC in the SLN. In contrast, rises in frequency of melanoma-reactive CD8 + $\mathrm{T}$ cells and NK cells, observed upon CpG-B ODN administration in both the SLN and the blood, were associated with the activation of SLN-resident plasmacytoid DC. These studies show that activation and recruitment of different DC subsets through specific cytokines and/or TLR-L can lead to (re)activation of tumorreactive $\mathrm{CD} 8+\mathrm{T}$ cells that appear to be already recruited to the SLN in early stages of melanoma development. These tumorreactive $\mathrm{T}$ cells may afford local as well as systemic control of metastatic outgrowth.

\section{P046}

\section{Toll-like receptor 9 and myeloid dendritic cells are essential for the activation of NK cells in cutaneous and visceral leishmaniasis}

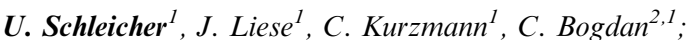 \\ ${ }^{I}$ Institute of Medical Microbiology and Hygiene, Freiburg, \\ Germany, ${ }^{2}$ Institute of Clinical Microbiology, Immunology \\ and Hygiene, Erlangen, Germany
}

NK cells contribute to the control of intracellular parasites including Leishmania. However, the cell types, pattern recognition receptors and cytokines required for the activation of NK cells in vivo are poorly defined.

Here, we show that Toll-like receptor (TLR) 9 is indispensable for NK cell IFN-gamma production and cytotoxicity in both cutaneous (L. major) and visceral leishmaniasis (L. infantum). In vitro, plasmacytoid dendritic cells (pDCs) released interferon (IFN)-alpha/beta and interleukin (IL)-12 in response to Leishmania promastigotes in a TLR9-dependent manner. In contrast, myeloid dendritic cells (mDCs) produced high amounts of IL-12, but not IFN-alpha/beta, in the presence of TLR9. Studies with IL-12- and IFN-alpha/beta receptor (IFNAR)deficient mice revealed that absence of IFN-alpha/beta signaling only slightly reduced the L. infantum-induced IFN-gamma production by NK cells, whereas NK cell IFN-gamma secretion and cytotoxicity was abolished in the absence of IL-12. The same phenotype was observed in mDC-depleted mice, whereas NK cell activation in response to $\mathrm{L}$. infantum remained intact after depletion of pDCs. Intracellular cytokine staining revealed that mDCs represent the source of IL-12 during the early phase of Leishmania infection. TLR9-deficient mice lacked IL-12 expression by mDCs after infection with L. major or L. infantum. Thus, NK cell priming in vivo in response to Leishmania parasites is strictly dependent on $\mathrm{mDCs}$, which sense the pathogen via TLR9 and subsequently deliver IL-12 to stimulate the NK cells.

\section{P047}

Poly(I:C) coated PLGA microparticles can induce dendritic cell maturation

\author{
D. R. Lorenzen ${ }^{1}$, C. Wischke ${ }^{2}$, J. Zimmermann ${ }^{3}$, H. H. Borchert ${ }^{2}$, \\ T. Nesselhut ${ }^{1}$, J. H. Peters ${ }^{3}$; \\ ${ }^{1}$ Institute for Tumortherapy, Duderstadt, Germany, ${ }^{2}$ Institute \\ for Pharmacy, Berlin, Germany, ${ }^{3}$ University Goettingen, \\ Goettingen, Germany
}

Dendritic cells (DC) initiate and regulate immune reactions and were used in clinical studies for cancer immunotherapy. Thereby, maturating DC are believed to be most effective for cancer vaccines, but the way of optimal stimulation of DC still has to be investigated. Immature DC generated from monocytes by GM-CSF + IL-4 can matured by Polyinosine-polycytidylic acid (poly I:C) as a ligand to the toll-like receptor 3 . The objective of this work was to prepare microparticles surface charged with poly $(\mathrm{I}: \mathrm{C})$ and to study wether those poly $(\mathrm{I}: \mathrm{C})$ coated particles are able to induce maturation of human monocyte-derived DC. Following phagocytosis of biodegradable microparticles, encapsulated tumor antigens might by released steadily, which may result in a long-lasting activation of DC and an optimized T-cell stimulation. Biodegradable microparticles with encapsulated model protein were prepared from poly (lactic-co-glocolic acid) (PLGA) using a static micro mixer for emulsification. PLGA microparticles were modified by DEAE -dextran to obtain cationic charged surfaces on which the anionic poly (I:C) was loaded by electrostatic interactions. The poly (I:C) coated microparticles were efficiently phagocytized by immature MoDC and induced differentiation into mature MoDC with upregulation of CD80 and CD86 and the maturation marker CD83. The phagocytosis of the unloaded particles did not lead to the maturation of dendritic cells and disproved the assumption, that phagocytosis of microparticles per se might induce maturation. Taken together, the use of poly (I:C) coated microparticles combined with encapsulated tumor antigens appears to be a promising concept for future DC-based cell therapies.

\section{Immune-regulatory Pathways}

\section{P048}

IDO-producing human dendritic cells: do they suppress the $\mathrm{T}$-cell response?

P. Terness, J. Chuang, S. Ehser, T. Bauer, I. Lahdou, M. Iancu, C. Kleist, H. Simon, S. Grimm, C. Christ, M. Hexel, G. Opelz; 


\section{Institute of Immunology, Heidelberg, Germany}

Studies in mice showed that the placental enzyme indoleamine-2,3dioxygenase (IDO) prevents T-cell mediated rejection of the fetus. Because IDO can also be expressed by dendritic cells the question arose whether IDO-DCs are T-cell-suppressive. Studies showed that a certain subpopulation of DCs (non-adherent, $\mathrm{CD} 123^{+}$, $\mathrm{CCR}^{+}{ }^{+}$cells) constitutively express IDO and strongly suppress Tcells. We generated such DCs but neither did they express IDO nor did they suppress T-cell responses (Blood 105:2480, 2005). Of course, this finding is not proof that constitutively IDO-DCs do not exist in humans; it only indicates that the described markers are not a "signature" for such a subpopulation (Trends Immunol $27: 68,2006)$. In the next experiment, we induced IDO by stimulating DCs with IFN- $\gamma$ and studied their action on allogeneic T cells or autoreactive MBP-specific T cells of multiple sclerosis patients. In neither situation were they suppressive. In a second series of studies we generated IDO-transgene expressing DCs. This time, we observed significant suppression of T-cell responses - an effect mediated by tryptophan metabolites (J Exp Med 196:447, 2002). The discrepant behaviors of IDO-transgene vs. natural IDOexpressing DCs led us to analyze the conditions for immunosuppression. Our observations indicate that the presence of stimulatory/suppressive cytokines and redox-active substances influence the function of IDO-DCs. Apparently, in certain microenvironments IDO-DCs are suppressive, whereas in others not. Because IDO-DCs are expected to have an outstanding importance for the pathogenesis of diseases, further research is merited towards elucidating under what conditions these cells play an immunoregulatory role in humans.

\section{P049}

\section{Immune-modulating effects of IL-13 blockade} in a mouse model of allergic lung inflammation

\author{
${\text { G. } \text { Grunig }^{1}, \text { E. Daley }}^{2}$, K. Robinson ${ }^{2}$; \\ ${ }^{1}$ Columbia University, New York, NY, USA,${ }^{2}$ St. Luke's Roosevelt \\ Hospital, New York, NY, USA
}

We have shown previously that inhibition of IL-13 in mice primed with allergen decreased the number of pulmonary antigen presenting cells that expressed high levels of MHCII and CD86. Because the immune response is thought to be regulated by the state of activation of dendritic cells, we hypothesized that transient blockade of IL-13 would induce a shift in T and B cell responses. Mouse B or T cells do not express an IL-13 receptor, therefore, immune-modulating effects of transient IL-13 blockade would likely be the result of a modification in antigen presentation. To test this hypothesis, wild type mice were immunized with Aspergillus antigen intraperitoneally and challenged with antigen intranasally for 3 times. IL-13 blocker or control antibody were administered during the time of the second and third intranasal challenges. The mice were rested for $2-3$ weeks and challenged once prior to analysis. The data show that the numbers of $\mathrm{T}$ cells capable of making IFNg were higher in lung draining lymph nodes of mice given IL-13 blockade relative to control mice. In addition, serum immunoglobulin levels were lower in mice that were given IL-13 blocker. Furthermore, the mice given IL-13 blocker developed significantly less remodeling lesions in the lungs as compared to mice given control protein. Together, our data suggest that targeting of a cytokine, like IL-13, that regulates the expression of molecules important for antigen presentation might be a strategy to regulate the immune response resulting in an amelioration of antigen-induced damage to the lungs.

\section{P050}

\section{Regulatory $\mathbf{T}$ cell depletion/blockade in combination with IL-21-based immunotherapy: a pre-clical study}

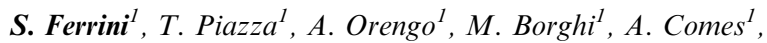 \\ M. Fabbi ${ }^{1}$, M. Colombo ${ }^{2}$; \\ ${ }^{1}$ Istituto Nazionale per la Ricerca sul Cancro, Genoa, Italy, ${ }^{2}$ Istituto \\ Nazionale Tumori, Milan, Italy
}

IL-21 is an immune-enhancing common-g chain cytokine. We found that, differently from IL-2, IL-21 is unable to induce naturally occurring or tumor-induced Treg cell expansion. To study the in vivo anti-tumor activities of IL-21, TS/A murine adenocarcinoma cells were genetically modified to secrete IL-21 (TS/AIL-21). Since TS/A-IL-21 cells were strongly immunogenic in syngeneic mice, we used them as therapeutic vaccine. In mice bearing TS/A parental cell (pc) micrometastases, vaccination with irradiated TS/A-IL-21 cells increased survival and cured $17 \%$ of mice. CD4 + CD25 + Foxp3 + Treg cells were present in TS/ Apc tumors and in draining LN, and IL-21 partially subverted their immunosuppressive activity in vitro at high concentrations. Co-administration of the TS/A-IL-21 vaccine with a depleting anti-CD25 mAb synergistically increased survival time and cured $80 \%$ of micrometastases-bearing mice through a CTL and IFNgamma-dependent mechanism. The combination of anti-CD25 $\mathrm{mAb}$ with low-dose rIL-21 (500 ng sc four times) also cured $57 \%$ of mice, while rIL-21 alone had limited effect. These treatments were highly effective if started early after metastasis induction ( 2 days) and then lost their efficacy. At day +4 after metastasis induction the vaccine + anti-CD25 mAb cured only $7 \%$ of mice, although survival was still increased. As a proportion tumorinfiltrating lymphocytes were CD25lowFoxp3 + /low they escaped from anti-CD25 mAb treatment. The addition of cyclophosphamide or of an anti-OX40 mAb (both blocking Treg functions) to the vaccine + anti-CD25 mAb treatment at day +4 allowed the cure of most mice. Altogether these data suggest that low-dose IL21 therapies can synergize with depletion or blockade of Treg cells.

\section{P051}

Prostaglandin E2 does not impair the immunostimulatory function of CD40-activated activated $B$ cells

\author{
A. Shimabukuro-Vornhagen ${ }^{1}$, T. Liebig ${ }^{1,2}$, A. Draube $^{1}$, \\ M. S. von Bergwelt-Baildon ${ }^{l}$; \\ ${ }^{1}$ Max-Eder Young Investigator Group, Molecular Tumor Biology \\ and Tumor Immunology, 1st Department of Internal Medicine, \\ University of Cologne, Cologne, Germany, ${ }^{2}$ Center for Molecular \\ Medicine of Cologne, University of Cologne, Cologne, Germany
}

Prostaglandin E2 (PGE2) is a potent immunomodulator that plays an important role in the regulation of the immune system in health and disease. It is a crucial maturation factor for dendritic cells (DC) and addition of PGE2 has been shown to improve the migration of DCs to the secondary lymphoid organs, a crucial step in the induction of an immune response. On the other hand it has been demonstrated that PGE2 can induce tolerogenic DCs. Production of PGE2 within the tumor microenvironment thus might be a potential challenge to the success of DC-based cancer vaccines in clinical trials.CD40-activated B cells (CD40-B) have been characterized as a promising alternative antigen-presenting cell for cellular cancer vaccines. We therefore investigated the influence of PGE2 on 
the phenotype, migration and antigen presenting function of CD40$\mathrm{B}$ cells. PGE2 did not influence migration towards the lymphoidhoming chemokines CCL21 and CXCL12 in vitro. Furthermore, we could not detect any changes in surface expression of HLA-DR or important costimulatory molecules such as CD80 and CD86. The ability of CD40-B cells to induce proliferation of allogeneic T cells was likewise not affected by culture of CD40-actived B cells in the presence of PGE2. Analysis of gene expression data demonstrated that none of the currently known PGE2 receptors was expressed upon CD40-activation of B cells. CD40-B cells are thus not susceptible to PGE2-mediated immunosuppression. They therefore seem to be useful in settings where PGE2 contributes to suppression of cancer vaccine-induced immune responses against tumors.

\section{P052}

\section{Induction of indoleamine 2,3-dioxigenase in dendritic cells during infection with Listeria monocytogenes and its implication for therapy}

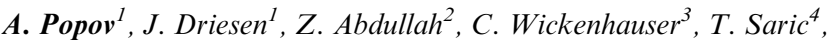 \\ S. Debey-Pascher ${ }^{1}$, M. Krönke ${ }^{2}$, O. Utermöhlen ${ }^{2}$, J. L. Schultze ${ }^{1}$; \\ ${ }^{I}$ Molecular Tumor Biology and Tumor Immunology at the Clinic I \\ for Internal Medicine, University of Cologne, Cologne, Germany, \\ ${ }^{2}$ Institute for Medical Microbiology, Immunology and Hygiene, \\ University of Cologne, Cologne, Germany, ${ }^{3}$ Institute for Pathology, \\ University of Cologne, Cologne, Germany, ${ }^{4}$ Institute for Neuro- \\ physiology, University of Cologne, Cologne, Germany
}

Attenuated avirulent strains of Listeria monocytogenes (L.m.) are often used as a vaccine vector in order to enhance the function of antigen-presenting cells and to induce inflammatory cytokine responses. Here we report the induction of dendritic cells (DC) with a regulatory phenotype following infection of human DC with different L.m. strains.

In vitro infection of human DC with wild type L.m. resulted in significant induction of the immunoregulatory enzyme indoleamine 2,3-dioxygenase (IDO) on transcriptional, protein and functional level. Moreover, enzymatically active IDO was induced in DC after infection with avirulent mutants (hly and prfA), whereas DC, treated with heat-killed L.m. were IDO-negative. In vivo, IDO-positive DC were revealed to be the major cellular component of the outer ring wall of suppurative granuloma in advanced human listeriosis. Induction of IDO in L.m.-infected DC was preceded by significant TNFalpha up-regulation and was triggered by TLR2 ligation. Using the clinically applied antiTNFalpha antibody infliximab we show that IDO induction is TNFalpha-dependent. Therefore, repression of IDO in granuloma-forming DC might be a major mechanism involved in exacerbation of granulomatous diseases during anti-TNFalpha therapy in rheumatoid arthritis. Moreover, due to possible induction of a regulatory DC phenotype, attenuated L.m. strains, should be tested for their IDO-inducing capability prior the use as vaccine vehicles or should be replaced by heat-inactivated L.monocytogenes preparations.

\section{P053}

\section{Myeloid derived "suppressor" cells activate natural killer cells to produce high levels of IFN- $\gamma$}

\author{
N. Nausch, A. Cerwenka; \\ German Cancer Research Center, Heidelberg, Germany
}

Accumulation of myeloid derived suppressor cells (MDSCs), which are characterized by the expression of Gr-1, CD11b, F4/80, CD115 and IL4R $\alpha$, has been observed in tumor-bearing mice, in cancer patients and in infection models. MDSCs isolated from tumor bearing mice or cancer patients potently suppress proliferation and cytokine production of T cells. So far, little is known about the interaction of MDSCs and NK cells.

In our study, we analyzed MDSC accumulation and function in a tumor model, in which MHC class I deficient NK cell sensitive RMA-S lymphoma cells were injected subcutaneously. We observed a significant accumulation of $\mathrm{Gr}-1^{+} \mathrm{F} 4 / 80^{+}$, $\mathrm{CD} 115^{+}$and IL4R $\alpha^{+}$MDSCs in blood and spleen of tumorbearing mice. Importantly, $\mathrm{Gr}-1^{+} \mathrm{F} 4 / 80^{+}$cells of tumor-bearing mice, but not control mice, expressed RAE-1, the ligand for the activating receptor NKG2D. Although MDSCs isolated from tumor bearing mice potently suppressed $\mathrm{T}$ cell proliferation, no effect on IL-2 stimulated NK cell proliferation or NK mediated cytotoxicity against YAC-1 targets was observed. Interestingly, $\mathrm{Gr}-1^{+} \mathrm{F} 4 / 80^{+}$positive cells isolated from tumor bearing mice significantly enhanced IFN- $\gamma$ production by NK cells. Moreover, IL-12 induced IFN- $\gamma$ production by NK cells was further increased by the presence of MDSCs from tumor bearing mice.

Our study identifies MDSCs as potent stimulators of NK cell mediated IFN- $\gamma$ production, which could have great impact on the design of improved vaccination protocols against cancer.

\section{P054}

\section{Activated $\mathbf{T}$ cells possess both killer and radiosensitizing properties in mixed cultures with tumor cells}

\author{
M. De Ridder ${ }^{1}$, V. N. Veronski ${ }^{1}$, K. Law ${ }^{2}$, D. Van den Berge ${ }^{1}$, \\ C. Monsaert ${ }^{2}$, G. A. Storme, \\ ${ }^{1}$ UZ Brussel, 1090, Belgium, ${ }^{2}$ Vrije Universiteit Brussel, 1090, \\ Belgium
}

Purpose: It is well known that polyclonal stimulation of $\mathrm{T}$ cells results in appearance of lymphokine-activated killers (LAK), which possess strong cytotoxicity against syngeneic tumor cells. The aim of this study was to examine whether LAK may also radiosensitize tumor cell through the release of IFN-gamma, a major activator of inducible nitric oxide synthase (iNOS).

Methods: Spleen cells from Balb/c mice were primed with bound anti-CD3 plus soluble anti-CD28 antibodies, expanded in the presence of IL-2 for 7 days and re-stimulated through CD3/ CD28. $\mathrm{T}$ cells $(\mathrm{CD} 4+$ and $\mathrm{CD} 8+)$ were selected by immunomagnetic MACS beads and analyzed for IFN-gamma by FACS and ELISA. Syngeneic EMT-6 tumor cells were incubated together with activated $\mathrm{T}$ cells for $16 \mathrm{~h}$ in hypoxia ( $1 \%$ oxygen), irradiated at 5-15 Gy, and examined for survival by colony formation assay.

Results: Activated $\mathrm{T}$ cells secreted $3000-5000 \mathrm{ng} / \mathrm{ml}$ of IFNgamma (40-70\% positive cells by FACS) compared with $0.1-$ $0.3 \mathrm{ng} / \mathrm{ml}$ of IFN-gamma ( $1-4 \%$ positive cells) in non-activated cells. In normoxia, activated T cells $\left(0.3-3 \times 10^{6} / \mathrm{ml}\right)$ displayed strong cytotoxicity in mixed cultures with EMT-6 tumor cells, while hypoxia, a common feature of solid tumors, significantly impaired this LAK effect. In contrast, the radiosensitivity of hypoxic EMT-6 tumor cells was significantly increased (1.4-1.6 times) and associated with the activation of iNOS. This effect was counteracted by the metabolic iNOS inhibitor aminoguanidine and by immunoneutralization of IFN-gamma. 
Conclusion: Activated $\mathrm{T}$ cells possess radiosensitizing activity in tumor relevant hypoxia, thus indicating a rationale for combining immunostimulatory and radiosensitizing strategies.

\section{P055}

\section{The radiosensitizing effect of immunoadjuvant OM-174 involves activation of inducible nitric oxide synthase through the interferon-gamma pathway}

V. N. Verovski ${ }^{1}$, M. De Ridder ${ }^{1}$, C. Chiavaroli ${ }^{2}$, D. Van den Berge ${ }^{1}, C$. Monsaert ${ }^{3}, K$. Law $^{3}$, G. A. Storme ${ }^{1}$; ${ }^{1}$ UZ Brussel, 1090, Belgium, ${ }^{2}$ OM, Geneva, Switzerland, ${ }^{3}$ Vrije Universiteit Brussel, 1090, Belgium

Purpose: To explore whether antitumor immunoadjuvant OM-174 can stimulate immune cells to produce interferon-gamma (IFNgamma) and thereby radiosensitize tumor cells.

Methods: Splenocytes from BALB/c mice were stimulated by OM174 at plasma-achievable concentrations $(0.03-3 \mu \mathrm{g} / \mathrm{ml})$, and afterward analyzed for the expression and secretion of IFNgamma by reverse transcriptase-polymerase chain reaction and enzyme-linked immunosorbent assay, respectively. Stimulated splenocytes were used as a source of IFN-gamma to radiosensitize hypoxic EMT-6 tumor cells through the cytokine-inducible isoform of nitric oxide synthase (iNOS)

Results: OM-174 activated the production of IFN-gamma at high levels that reached $70 \mathrm{ng} / \mathrm{ml}$ in normoxia ( $21 \%$ oxygen) and $27 \mathrm{ng} /$ $\mathrm{ml}$ in tumor-relevant hypoxia ( $1 \%$ oxygen). This caused up to $2.1-$ fold radiosensitization of EMT-6 tumor cells, which was associated with the iNOS-mediated production of the radiosensitizing molecule nitric oxide, as confirmed by accumulation of its oxidative metabolite nitrite, Western blot analysis, and reverse transcriptasepolymerase chain reaction. Both iNOS activation and radiosensitization were counteracted by neutralizing antibodies against IFNgamma. The same mechanism of radiosensitization through the IFN-gamma secretion pathway was identified for IL-12 + IL-18, which are known to mediate IFN-gamma responses. Hypoxia displayed a dual effect on the immune-tumor cell interaction, by downregulating the expression of the IFN-gamma gene while upregulating iNOS at transcriptional level.

Conclusion: Immunoadjuvant $\mathrm{OM}-174$ is an efficient radiosensitizer of tumor cells through activation of the IFN-gamma secretion pathway in immune cells. This finding indicates a rationale for combining immunostimulatory and radiosensitizing strategies and extends the potential therapeutic applications of OM-174

\section{Basic Mechanisms}

\section{P056 \\ Inhibition of BCR-ABL activity affects the generation of CTL responses against CML}

K. M. Brauer, D. Werth, K. von Schwarzenberg, A. Bringmann, L. Kanz, F. Grünebach, P. Brossart

Abteilung für Hämatologie, Onkologie, Immunologie, Rheumatologie und Pulmologie, Universitätsklinikum Tübingen, Tübingen, Germany

Chronic myelogenous leukemia (CML) is a myeloproliferative disorder caused by excessive granulopoiesis due to the formation of the constitutively active tyrosine kinase BCR-ABL. An effective drug against $C M L$ is imatinib mesylate, a tyrosine kinase inhibitor acting on Abl kinases, c-KIT and platelet-derived growth factor receptor. Recently, a study revealed, that patients treated with imatinib showed impaired CTL responses in comparison to patients treated with IFN- $\alpha$, which might be due to a treatment induced reduction in immunogenicity of CML cells or immunosuppressive effects.

In our study we found that inhibition of BCR-ABL using imatinib leads to a reduced immunogenicity of CML cells. By treating CML cells with imatinib we could show that the resulting inhibition of BCR-ABL leads to a decreased expression of tumor antigens including survivin, adipophilin, hTERT, WT-1, Bcl-xL and $\mathrm{Bcl}-2$ in correlation to a decreased development of $\mathrm{CML}$ specific CTLs. In contrast, this reduction in immunogenicity was not observed when a CML cell line resistant to the inhibitory effects of imatinib was utilized, but could be confirmed by imatinib treatment of primary CML cells. Depletion of BCR-ABL expression using specific siRNA against BCR-ABL further confirmed the direct influence of the kinase activity on the inducibility of CML-specific CTL.

These results indicate that BCR-ABL substantially contributes to the immunogenicity of CML cells, probably due to upregulation of immunogenic antigens by its uncontrolled tyrosine kinase activity. Imatinib treatment is therefore likely to interfere with immunotherapeutic approaches due to an inhibition of the priming of CTL responses in patients.

\section{P057}

\section{A prostate carcinoma antigen reveals a novel cytosolic antigen processing pathway for ER-targeted proteins}

\author{
E. Schlosser ${ }^{1}$, C. Otero ${ }^{1}$, D. Legler ${ }^{2}$, M. Groettrup ${ }^{1}$; \\ ${ }^{1}$ University of Constance, Konstanz, Germany, \\ ${ }^{2}$ Biotechnology Institute Thurgau, Kreuzlingen, Switzerland
}

Proteins that bear an N-terminal endoplasmic reticulum leader peptide are inserted into the lumen of the endoplasmic reticulum followed by cleavage of the signal peptide and glycosylation. Several major histocompatibility complex class I restricted $\mathrm{T}$ cell epitopes can be generated from these glycoproteins by the proteasome after their retrotranslocation from the endoplasmic reticulum into the cytosol. In this study we show that an HLA-A*0201 restricted T cell epitope from prostate stem cell antigen, that is recognized by prostate carcinoma specific cytotoxic T lymphocytes, encompasses the cleavage site of the endoplasmic reticulum signal peptidase. This cleavage yields truncated peptides that fail to bind to HLA-A*0201 and to be recognized by cytotoxic $\mathrm{T}$ lymphocytes. Since processing of prostate stem cell antigen by signal peptidase occurs immediately after cotranslational insertion, the epitope must be synthesized and processed from polypeptides that have never reached the endoplasmic reticulum. The processing of this epitope depends on the activity of the proteasome and the transporter associated with antigen processing and reveals a novel pathway of class I processing that relies on the failure of endoplasmic reticulum targeted proteins to reach their compartment of destiny.

\section{P058}

\section{The CD83 reporter mouse}

M. J. Lechmann, A. Wakeham, T. W. Mak; The Campbell Family Institute for Breast Cancer Research, Toronto, ON, Canada 
For over a decade, the glycoprotein CD83 has been known to be one of the best markers for mature DC. And despite of intensive investigations by different groups the functions of this conserved molecule remains in the twilight of controversial discoveries. In thymus, CD83 is essential for the generation of CD4 $+\mathrm{T}$ cells. And though, CD83 deficient DC are not impaired in their stimulatory capacity, soluble CD83, which can be generated by splice forms in humans or by proteolytic cleavage from activated DC and B cells, showed strong immunosuppressive roles. Various cell types including thymic epithelial cells in mouse, activated T, B and DCs are reported to express CD83 on their surface, while others are named to have transient or solely intracellular expression. Even more confusing is the search for the unknown CD83 ligand. CD83 targets are referred to be on activated T cells, B cells, im and $\mathrm{mDCs}$ and monocytes.

Because all this suggest a universal role for CD83 in immune function, we generated a CD83 reporter mouse. Unfortunately, the specific $\mathrm{CD} 83$ promotor is unknown.

To avoid any possible functional influence interfering CD83 expression, the EGFP reporter gene has been linked over an Internal Ribosomal Entry Site right after the stop codon of the CD83 gene. With those mice we characterize the CD83 expression on the different cell types in vivo and ex vivo and establish a mouse model using the CD83 gene as marker to observe activation status and migration of DC and lymphocytes upon different inflammatory stimuli.

\section{P059}

\section{Recruitment of monocyte subpopulations by leukocytapheresis}

\author{
M. Hendelmeier ${ }^{1}$, J. Bruegel ${ }^{1}$, M. Erdmann ${ }^{2}$, E. Strasser ${ }^{1}$ \\ R. Eckstein ${ }^{1}$; \\ ${ }^{1}$ Division of Transfusion Medicine, Erlangen, Germany \\ ${ }^{2}$ Department of Dermatology, Erlangen, Germany
}

Background: Monocyte-derived Dendritic Cells (DC) are promising tools for the cellular therapy of malignant melanoma. Monocytes are, however, immune competent cells and consist of distinct subsets with different physiological roles. Leukocytapheresis is a stimulus for recruitment of distinct monocyte subpopulations.

Materials and methods: Healtyh blood donors $(n=22)$ unterwent leukocytapheresis. Monocyte subpopulations in blood counts and in the monocyte apheresis product (MAP) were analyzed by flow cytometry (FACS Calibur, BD). The impact of the apheresis procedure was demonstrated by calculation of the recruitment factor (RF).

Calculation formula: $\mathrm{RF}=$ (postdonation cell count + cell yield)/predonation cell count.

Results: Significant differences between the RF of CD14++ monocytes and CD14 + CD16 + monocytes could be demonstrated $(P=0.003) . \quad \mathrm{CD} 14+\mathrm{CD} 16+$ monocytes and CD33dimCD16 + monocytes showed a significant enrichment in MAP (both $P<0.001$ ). The calculated RF of CD33bright monocytes differed significantly from the RF of CD14 + CD16+ monocytes $(P=0.003)$ and $\mathrm{CD} 33 \operatorname{dimCD} 16+$ monocytes $(P=0.0016)$. Between the RF of CD14 $+\mathrm{CD} 16+$ monocytes and the RF of CD33dimCD16 + monocytes no significant difference was detectable.

Conclusion: The different recruiting pattern of monocyte subpopulations indicates an immune intervention by leukocytaph- eresis. The understanding of monocyte heterogeneity and the impact of leukocytapheresis on monocyte subpopulations may have implications for the development of cellular therapies with DC.

\section{P060}

\section{MLN51 and GM-CSF involvement in fibroblast-like synoviocytes (FLS) proliferation in the pathogenesis of rheumatoid arthritis}

\author{
M. Kang ${ }^{l}$, D. $\operatorname{Lim}^{l}, Y . B a e^{1,2}$; \\ ${ }^{1}$ Division of DC Immunotherapy, CreaGene Research Institute, \\ Seongnam-si, Gyeonggi, Republic of Korea, ${ }^{2}$ Department of \\ Biological Science, Sungkyunkwan University, Seongnam-si, \\ Gyeonggi, Republic of Korea
}

Rheumatoid arthritis (RA) is an inflammatory autoimmune disease of unclear etiology. This study was conducted to identify critical factors involved in the synovial hyperplasia in RA pathology. We applied cDNA microarray analysis to profile the gene expressions of RA fibroblast-like synoviocytes (FLSs) from patients with RA. We found that the MLN51 (metastatic lymph node 51) gene, identified in breast cancer, is remarkably upregulated in the hyperactive RA FLSs. However, growth retarded RA FLSs passaged in vitro expressed small quantities of MLN51. MLN51 expression was significantly enhanced in the FLSs when the growth-retarded FLSs were treated with granulocyte-macrophage colony-stimulating factor (GM-CSF) or synovial fluid (SF). Anti-GM-CSF neutralizing antibody blocked the MLN51 expression even though the FLSs were cultured in the presence of SF. In contrast, GM-CSF in SFs existed at a significant level in the patients with RA $(n=6)$, in comparison with the other inflammatory cytokines, IL- $\beta$ and TNF- $\alpha$. Most RA FLSs at passage 10 or more recovered from their growth retardation when cultured in the presence of SF. The SF-mediated growth recovery was markedly impaired by anti-GM-CSF antibody. Growth-retarded RA FLSs recovered their proliferative capacity after treatment with GM-CSF in a dose-dependent manner. However, MLN51 knock-down by siRNA completely blocked the GM-CSF/SF-mediated proliferation of RA FLSs. Taken together, our results imply that MLN51, induced by GMCSF, is important in the proliferation of RA FLSs in the pathogenesis of RA.

\section{P061 \\ Investigation of TNF effects on the hematological cell lines}

\author{
V. Jurisic ${ }^{1}$, V. Kojic ${ }^{2}$, D. Jakimov ${ }^{2}$, G. Bogdanovic ${ }^{2}$; \\ ${ }^{1}$ University of Kragujevac, Kragujevc, Serbia and Montenegro, \\ ${ }^{2}$ Institute of Oncology, Novi Sad, Serbia and Montenegro
}

Tumor necrosis factor (TNF)-alpha, a pleiotropic cytokine, has been shown to induce mostly apoptosis in sensitive cells. We here investigated its in vitro dose- and time-related effect on three different hematological cell lines, including erytroleukemia, K562 cells; B-cell line Raji, derived from Burkitt lymphoma patients; and novel cell line derived from MDS patients, therapy induced. The effects were determined at different intracellular levels. The membrane alteration was estimated by lactate dehy- 
drogenase (LDH) release, by flow cytometry (using annexin V and Propidium Iodide) and on morphology. The intracellular metabolic energy analyzed by the total intracellular enzyme activity after cell sonification, and the total cytosole protein mass by sulforhodamine B assay. The cell growth was estimated by incorporation of $[(3) \mathrm{H}]$ thymidine into DNA. Significant increase of $\mathrm{LDH}$ through cell membrane alteration was accompanied by decrease of intracellular metabolized energy and total protein mass. Effect of apoptosis or cell proliferation was diferent on cell type. In addition, TNF-alpha at lower concentrations (125 and $250 \mathrm{pg} / \mathrm{ml}$ ) significantly induced cell proliferation in Raji cells, compared to $1.000 \mathrm{pg} / \mathrm{ml}$ of TNF-alpha, which induced more cell death. TNF-alpha induced different rate of apoptosis that depending on cell type and level of TNF receptor molecule expression. Here we also correlated the rate of apoptosis in respect to TNF receptor 1 (TNFR-1) and TNFR-2 type on these cells and we found that TNFR1 is more asssociated with high rate of apoptosis while TNFR 2 is more associtated with cell prolifferation.

\section{P062 \\ Antigen processing via MHC-class II pathway in CD40-activated B cells: apoptotic or necrotic tumor cells as a source of antigen}

E. Kondo, M. von Bergwelt-Baildon;

Max-Eder Young Investigator Group, Molecular Tumor Biology and Tumor Immunology, 1st Department of Internal Medicine, University of Cologne, Cologne, Germany

Antigen loading of APC for vaccination frequently relies on the use of molecularly defined antigen such as peptides or RNA. Tumor cells, however, are an intriguing alternative as they contain unknown, relevant antigens and are immunogenic when rendered necrotic or apoptotic. We have previously demonstrated that CD40-activated B cells (CD40-Bs) are an interesting cellular adjuvant due to their efficient antigen presentation and expansion. We have previously established antigen loading using HLA-A*0201 binding peptides in order to induce primary $\mathrm{T}$ cell responses. Nevertheless, it remains unclear how CD40-Bs process exogenous antigen via MHC class-II pathway and whether they can be successfully antigen loaded using tumor cells.

We generated $\mathrm{CD} 4{ }^{+} \mathrm{T}$ cell lines specific for a HLA-DRB $1 * 0101$ binding CMVpp65 epitope (KYQEFFWDANDIYRI) and cloned them by limiting dilution. Using step-by-step blockade of MHC class-II processing we demonstrated that in CD40-Bs classical and alternative pathways are operative. Next, we compared antigen presentation of CD40-Bs loaded with surrogate tumor: K562 cells transfected with CMV-pp65 or EGFP were induced to undergo apoptosis or necrosis and co-incubated with CD40-Bs for $6 \mathrm{~h}$. In an ELISpot assay, the $\mathrm{CD} 4^{+}$clone recognized both cell preparations specifically. However, CD40-Bs were more efficiently recognized when necrotic cells were used. Since whole tumor cell ingesting CD40-Bs could not be detected in FACS, CD40-Bs seemed to incorporate released antigens from dead cells. Correspondingly CD40-Bs took up Lucifer yellow (pinocytosis) comparable to DC, but less Dextran- or Albumin-FITC (receptor mediated endocytois). Taken together, CD40-Bs efficiently process and present antigen from necrotic cells via MHC class-II.
P063

Susceptibility of CD16 + CD56dim NK cells to reactive oxygen species produced byactivated granulocytes due to low antioxidative capacity

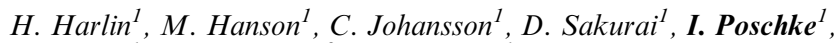
H. Norell ${ }^{1}$, K. Malmberg ${ }^{2}$, R. Kiessling ${ }^{1}$;

${ }^{1}$ Cancer Center Karolinska, Deparment of Oncology and Pathology, Stockholm, Sweden, ${ }^{2}$ Center for Infectious

Medicine, Department of Medicine, Stockholm, Sweden

Human NK cells can be divided into CD56dim and CD56bright subsets. These two types of NK cells respond to different types of stimuli, with CD56dim NK cells having direct cytotoxic ability and CD56bright NK cells having mainly immunoregulatory function. We show that the CD16 + CD56dim NK subset is characterized by sensitivity to cell death induced by activated granulocytes. We identified hydrogen peroxide $(\mathrm{H} 2 \mathrm{O} 2)$ as the major effector molecule responsible for the cytotoxic effect of granulocytes on CD56dim NK cells, since the ability of granulocytes to kill CD56dim NK cells was completely abrogated in the presence of the hydrogen peroxide scavenger catalase. When exposing NK cells to $\mathrm{H} 2 \mathrm{O} 2$, CD56dim cells showed rapid mitochondrial depolarization and downregulation of activating NK receptors, eventually resulting in cell death, whereas CD56bright cells remained unaffected. The difference in sensitivity to $\mathrm{H} 2 \mathrm{O} 2$ was mirrored by a difference in intracellular oxidation levels between CD56dim and CD56bright NK cells, and cell lysates from the latter subset possessed a greater ability to block $\mathrm{H} 2 \mathrm{O} 2$ mediated oxidation. Our data may explain the preferential accumulation of CD56bright NK cells often seen in environments rich in reactive oxygen species (ROS), such as at sites of chronic inflammation and in tumors.

\section{P064 \\ Interactions of $\mathbf{T}$ cells with activated $\mathbf{B}$ cells are dynamic, short-lived, and promiscuous}

\begin{abstract}
N. Klein-González ${ }^{1,2}$, S. Balkow ${ }^{3}$, E. Kondo ${ }^{1}$, S. Grabbe $^{4}$, M. von Bergwelt-Baildon ${ }^{1}$;

${ }^{1}$ Max Eder Young Investigator Group, Molecular Tumor Biology and Tumor Immunology, 1st Department of Internal Medicine, University of Cologne, Cologne, Germany, ${ }^{2}$ Center for Molecular Medicine of Cologne, University of Cologne, Cologne, Germany, ${ }^{3}$ Department of Dermatology, University of Münster, Münster, Germany, ${ }^{4}$ Department of Dermatology, University of Essen, Essen, Germany
\end{abstract}

We have previously introduced an alternative cellular adjuvant that could be integrated into DC-based immunotherapy. B cells when activated via CD40-L/IL-4 (CD40-B) can be expanded from small amounts of peripheral blood in 12-14 days. At day 14 these cells are $>95 \% \mathrm{CD}_{20}{ }^{+}$and $\mathrm{CD} 80 / 83 / 86 / \mathrm{MHCI} / \mathrm{MHCII}{ }^{\mathrm{hi}}$. CD40-Bs take up antigen independent of the B-cell receptor via endocytosis, process it via the classical and an alternative MHC II pathway and prime naïve T cells. They have a doubling time of $3-$ 4 days and can be used to efficiently expand $\mathrm{CD} 4^{+}$and $\mathrm{CD} 8^{+} \mathrm{T}$ cells in a highly differentiated in vitro system. Nevertheless, it remains unclear whether such cells have the property to colocalize and interact with $\mathrm{T}$ cells in a physiological context. 
Here, we show that CD40-Bs express the full lymph node homing triad including CD62L, CCR7/CXCR4 and LFA1. Murine and human CD40-Bs migrate towards cognate ligands such as CCL19, CCL21 and CXCL12. Furthermore, such CD40Bs express several $\mathrm{T}$-cell chemoattractants and induce $\mathrm{T}$-cell chemotaxis in vitro.

To dissect T-cell/APC interaction on a single cell we analyzed three-dimensional migration in collagen matrix. Interestingly, antigen-loaded CD40-Bs differ from immature and mature DC by displaying a rapid migratory pattern undergoing promiscuous, short-lived $(7.5 \mathrm{~min})$ but stable interactions with cognate $\mathrm{T}$ cells. Taken together, these data suggest that CD40-Bs are equipped with the receptors and migratory capacity necessary to home to secondary lymphoid organs and have the property to attract and enter into stable contacts with $\mathrm{T}$ cells.

\section{P065 \\ Mast cells are an essential hematopoietic component to polyp development}

\section{K. Khazaie; \\ Dana Farber Cancer Institute, Boston, MA, USA}

It is generally agreed that most colon cancers develop from adenomatous polyps and it is this fact upon which screening strategies are based. Although there is overwhelming evidence to link intrinsic genetic lesions with the formation of these preneoplastic lesions, recent data suggest that the tumor stromal environment also plays an essential role in this disease. In particular, it has been suggested that "CD34 immature myeloid precursors" are required for tumor development and invasion. We have used mice conditional for the stabilization of $\beta$-catenin or defective for the adenomatous polyposis coli (APC) gene to investigate the identity and importance of tumor infiltrating hematopoietic cells in polyposis. We show that, from the onset, polyps are infiltrated with pro-inflammatory mast cells and their precursors. Depletion of mast cells either pharmacologically or through the generation of chimeric mice with genetic lesions in mast cell development leads to a profound remission of existing polyps. Our data suggest that mast cells are an essential hematopoietic component to preneoplastic polyp development and are a novel target for therapeutic intervention.

\section{P066 \\ Interferon regulatory factor (IRF)-1 is a key player in the cross-talk between tolerogenic dendritic cells and CD4 + CD25 + regulatory $T$ cells.}

L. Gabriele, A. Fragale, P. Borghi, E. Stellacci, R. Ilari, A. Lanciotti, A. Remoli, M. Venditti, F. Belardelli, A. Battistini; Istituto Superiore di Sanità, Roma, Italy

Regulatory T cells (Treg) and tolerogenic dendritic cells (DCs) are instrumental in the maintenance of peripheral immune tolerance and the control of adaptive immune responses. However, the molecular mechanisms underlying the induction of immune tolerance remain elusive. In this context, transcription factors which belong to the IFN-regulatory factor (IRF) family are emerging as master regulators of signalling crucially affecting many aspects of the innate and adaptive immune responses.

Here, we report data highlighting a new role of IRF-1 in regulating immune tolerance mechanisms triggered by the crosstalk between tolerogenic DC and CD4 + CD25+ Treg. IRF-1 deficient mice $\left(\mathrm{IRF}_{-} \mathrm{1}^{-/}\right)$exhibited a tolerogenic-polarized immune system characterized by two prominent features. The first is a defective development of DCs with predominance of immature and tolerogenic populations, such as plasmacytoid DC (pDCs), expressing high levels of IL-10, and unable to undergo full maturation following virus infection both ex vivo and in vivo. Accordingly, DCs from IRF-1 ${ }^{-/}$mice were less efficient in stimulating the proliferation of allogeneic $\mathrm{T}$ cells and instead induced an IL-10-mediated suppressive activity in allogeneic $\mathrm{CD} 4{ }^{+} \mathrm{CD} 25^{+}$Treg cells. The second is a selective increase in $\mathrm{CD} 4{ }^{+} \mathrm{CD} 25^{+}$Foxp $^{+}$Treg cells showing tolerogenic phenotype and high suppressor activity. These findings uncover IRF-1 as an essential regulator of DC and Treg homeostasis, thus opening new perspectives for the control of diseases associated with break of tolerance.

\section{DC-modulation}

\section{P067}

IgG-opsonization of HIV impedes provirus formation in and infection of DCs and subsequent long-term transfer to $\mathrm{T}$ cells

\author{
D. Wilflingseder ${ }^{1}$, Z. Banki ${ }^{1}$, E. Garcia ${ }^{2}$, M. Pruenster ${ }^{1}$, \\ B. Muellauer ${ }^{1}$, C. G. Ammann ${ }^{1}$, M. P. Dierich ${ }^{1}$, V. Piguet ${ }^{2}$, \\ H. Stoiber ${ }^{l}$; \\ ${ }^{1}$ Innsbruck Medical University, Innsbruck, Austria, ${ }^{2}$ University \\ Hospital and Medical School of Geneva, Geneva, Switzerland
}

Already at initial phases of infection, HIV is coated with complement fragments. During the chronic phase, when HIV-specific IgGs appear, the virus circulates immune-complexed with $\mathrm{IgG}$ and complement. Thus, we studied the interaction of DCs and DC-T cell co-cultures with complement (C)-opsonized and C-IgGopsonized HIV. HIV-infection of monocyte-derived DCs and circulating BDCA-1-positive DCs was significantly reduced upon the presence of virus-specific but non-neutralising IgGs. DCs exposed to C-Ig-HIV or IgG-opsonized HIV showed an impaired provirus formation and p24 production and a decreased transmission rate to autologous non-stimulated $\mathrm{T}$ cells upon migration along a chemokine gradient. This reduced infectivity was also observed in long-term experiments, when $\mathrm{T}$ cells were added delayed to DCs exposed to IgG-coated HIV without migration. Similar kinetics were seen when sera from HIV-1-infected individuals before and after seroconversion were used in infection assays. Both, complement- and C-IgG-opsonized HIV were captured and targeted to a tetraspanin-rich endosome in iDCs, but differed with respect to MHC class II co-localization. The reduced infection by IgG-opsonized HIV is possibly due to interactions of virus bound IgG with FcgammaRIIb expressed on DCs. Therefore, the intracellular fate and transmission of immune-complexed HIV seems to differ depending on time and opsonization pattern. 


\section{P068}

\section{Sorafenib but not sunitinib affects function of dendritic cells and induction of primary immune responses}

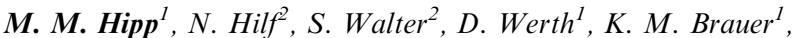 \\ T. Weinschenk ${ }^{2}, H$. Singh-Jasuja ${ }^{2}$, P. Brossart ${ }^{1}$; \\ ${ }^{1}$ Abteilung für Hämatologie, Onkologie, Immunologie, \\ Rheumatologie und Pulmologie, Universitätsklinikum Tübingen, \\ Tübingen, Germany, ${ }^{2}$ Immatics Biotechnologies GmbH, Tübingen, \\ Germany
}

The tyrosine kinase inhibitors sorafenib and sunitinib are approved for the treatment of patients with malignant diseases. To analyze the possible use of these compounds in combination with immunotherapeutic approaches we analyzed the effects of both inhibitors on the immunostimulatory capacity of human DCs and the induction of primary immune responses in vivo. Sorafenib but not sunitinib inhibits function of DCs, characterized by reduced secretion of cytokines and expression of CD1a, MHC and costimulatory molecules in response to TLR ligands, as well as by their impaired ability to migrate and stimulate T-cell responses. These inhibitory effects are mediated via inhibition of $\mathrm{PI} 3$ and MAP kinases and $\mathrm{NF} \kappa \mathrm{B}$ signaling. In contrast, sorafenib had no influence on the phenotype and proliferation of $\mathrm{T}$ cells.

To analyze the effects of both TKIs on CTL induction in vivo, C57BL/6 mice were pretreated with sorafenib or sunitinib and immunized with OVA257-264 peptide. Sorafenib application, but not sunitinib, significantly reduced the induction of antigen specific $\mathrm{T}$ cells. Interestingly, numbers of CD4 + CD25 + regulatory $\mathrm{T}$ cells were reduced in PBMCs from mice treated with sunitinib. In summary, our results indicate that sunitinib, but not sorafenib is suitable for combination with immunotherapeutic approaches for treatment of cancer patients.

\section{P069 \\ Modulation of dendritic cells as intervention strategy in type 1 diabetes}

W. W. J. Unger, S. Laban, B. O. Roep; LUMC, Leiden, The Netherlands

Type 1 diabetes is characterized by T-cell mediated autoimmune destruction of the pancreatic beta cells. Current intervention strategies, targeting T-cells, comprise the use of broad-range immunosuppressants causing considerable non-specific sideeffects. Ideally, immune intervention should be specifically tailored towards the diabetogenic T-cells. Since dendritic cells (DC) are able to orchestrate $\mathrm{T}$-cell responses our aim is to assess whether vaccination with modulated DC can be used as intervention tool in newly diagnosed T1D patients or longstanding T1D patients who were transplanted with pancreatic islets.

Treatment of DC with either dexamethasone (DEX-DC) or the active form of vitamin $\mathrm{D}(3), 1 \alpha, 25$-dihydroxyvitamin $\mathrm{D}(3)$ (D3-DC) renders these cells in a semi-mature state even upon lipopolysaccharide (LPS)-induced maturation. Both treatments abrogated the ability to secrete IL-12p70, but not IL-10, and affected the T-cell stimulatory function. Moreover, priming of naïve T-cells with D3-DC led to the induction of regulatory T-cells, which are able to suppress the activation and proliferation of responder T-cells.

D3-DC, but not DEX-DC, have a higher expression of molecules related to the induction of apoptosis than non-treated DC, which corroborates with our previous finding that D3-DC selectively induce apoptosis in committed T-cells. Importantly, modulation with either DEX or D3 arrests DC in a stable, tolerogenic mode, as their phenotype remains unaltered when encountering a pro-inflammatory signal even 5 days after maturation.

Together, these findings suggest that both DEX-DC and D3-DC possess durable tolerogenic features and are potentially useful to specifically down-regulate unwanted immune responses and induce immune tolerance in T1D patients.

\section{P070}

\section{VSV-G improves the efficacy of exosome-based, DC-targeted vaccines}

\author{
V.V. Temchura ${ }^{1}$, S. Kuate ${ }^{2}$, K. Überla ${ }^{1}$; \\ ${ }^{1}$ Department of Molecular and Medical Virology, Bochum, \\ Germany, ${ }^{2} \mathrm{NCI} / \mathrm{NIH}$, Bethesda, MD, USA
}

Exosomes released from dendritic cells (DC) or cancer cells have been proposed as candidates for immunotherapeutic vaccination. Based on previous observations with virus-like particles (Kuate et al. 2006), we attempted to improve exosomal vaccines by incorporation of the fusion G-protein of vesicular stomatitis virus (VSV-G) into the membrane of exosome-like vesicles (ELV) derived from 293T cells. VSV-G is known to mediate entry into DC and might thus allow more efficient stimulation of immune responses. Indeed, In vitro tests showed that VSV-G mediated enhancement of ELV internalization by murine splenic DC. Although the ELV could themselves moderately activate DC, VSV-G enhanced the capability to induce acute phenotypic (upregulation of co-stimulatory molecules surface expression) and functional (production of IL-12p70) maturation. Using ovalbumin (OVA) anchored in the membrane of ELV as a model antigen we observed that co-expression of VSV-G and OVA on the same vesicles (G-OVA-ELV) led to a 100 -fold increase of antigen crosspresentation in vitro. DC processed these ELV through a degradation mechanism that involves endosomal acidification. In vivo vaccination with G-OVA-ELV increased the serum level of antiOVA IgG2a antibodies, expanded TCR-specific CD8 T cells and provided strong protection against challenge with OVA expressing tumor cells in mice.

\section{P071}

\section{Cytotoxic $\mathbf{T}$ lymphocytes regulate the ability of dendritic cells to induce anti-tumor immune responses}

\section{J. Yang, K. Andrew, J. Qin, R. Perret, F. Ronchese,} Malaghan Institute of Medical Research, Wellington, New Zealand

Immunization with dendritic cells (DC) loaded with tumor peptides is an effective method to induce tumor-specific CTL. However, DC that present tumor peptide on MHC I molecules may also become targets of tumor-specific CTL.

We examined the survival of DC in vivo during the induction of anti-tumor immune responses. We labeled DC with fluorescent dyes, loaded them with tumor peptides, and examined their ability to accumulate in the draining lymph node after subcutaneous injection. DC injected into naive animals migrated to the draining lymph node, where they remained for several days regardless of 
whether they were loaded with antigen or not. In contrast, DC loaded with tumor antigen and injected into immune mice were killed by $\mathrm{CD} 8+\mathrm{T}$ cells, and failed to accumulate in the draining lymph node. Killing of DC was perforin dependent, as DC survived in immune perforin-deficient mice. Treatment with LPS was not sufficient to make DC resistant to CTL-mediated killing in vivo.

To determine whether DC killing impacts on their ability to recall immune responses, we compared the ability of activated PKO and normal T cells to expand and mediate tumor protection after DC boost. DC boost induced expansion of activated PKO $\mathrm{CD} 8+\mathrm{T}$ cells, and considerably improved their ability to mediate tumor protection. In contrast, normal $\mathrm{CD} 8+\mathrm{T}$ cells only expanded modestly after DC boost, and their ability to mediate tumor protection was only moderately improved. We conclude that CTL-mediated elimination of DC affects their ability to optimally boost anti-tumor immune responses.

\section{P072}

\section{Stability and functional heterogeneity of monocyte-derived dendritic cells}

\author{
P. Gogolák, B. Réthi, I. Szatmári, B. Dezső, L. Nagy, \\ É. Rajnavölgyi; \\ University of Debrecen, Debrecen, Hungary
}

CD14 ${ }^{\text {high }}$ monocytes cultured in serum free AIMV medium with GM-CSF and IL-4 differentiate simultaneously to CD $14{ }^{\text {low }} \mathrm{CD} 1 \mathrm{a}-$ and CD14-CD1a + dendritic cells (moDC). These co-existing moDC subsets possess similar migratory and T-lymphocyte activating potential but differ in their internalizing capacity, cytokine and chemokine profile and T-lymphocyte polarizing activity. The ratio of CD1-cells could be increased by serum lipoproteins in a dose dependent manner, whereas depletion of serum lipids skewed moDCs differentiation to CDla + cells. Immature CD1a-cells differentiate to CDla + moDC, but activation by various stimuli blocks this process. PPAR $\gamma$ was shown to regulate CD1 expression and high phagocytic activity and limited IL-12 production has been attributed to CD1a- cells generated in the presence of $\operatorname{PPAR} \gamma$ ligands. Real time RT-PCR measurements showed higher expression of functionally active PPAR $\gamma$ and their target genes in CD1amoDC than in CD1a + cells. Hence, lipoprotein-mediated regulation of moDC differentiation acts through the activation of PPARg by endo- and/or exogenous agonists and modulates CD1a expression. The ratio of CD1a- and CD1 + moDC derived from monocytes of various donors showed high variability pointing to the modulatory role of lipid environment on moDC differentiation, CD1a dichotomy, activation state and consequently the functional potential of both immature and mature moDCs. CDla + PPARgand CD1-PPARg + moDCs were detected as distinct cell types in peripheral lymphoid tissues and in the lung. In vitro manipulation of the PPAR $\gamma$ mediated regulatory pathway and termination of $\mathrm{CD} 1 \mathrm{a}-$ to $\mathrm{CD} 1 \mathrm{a}+$ transition by various activation signals opens up new possibilities in optimizing moDCs conditioning for immunotherapy.

\section{P073 \\ Impact of IL-3 derived human dendritic cells expressing IDO on allogeneic and antigen-specific T-cell responses}

\author{
S. Löb ${ }^{1,2}$, M. Deeg ${ }^{3}$, R. Schäfer ${ }^{4}, H$. Rammensee $^{1}$, \\ A. Königsrainer ${ }^{2}$;
}

${ }^{1}$ Interfacultary Institute of Cell Biology, Tübingen, Germany, ${ }^{2}$ Department of General-, Visceral- and Transplantsurgery, Tübingen, Germany, ${ }^{3}$ Department of Internal Medicine, Tübingen, Germany, ${ }^{4}$ Institute of Clinical and Experimental Transfusion Medicine, Tübingen, Germany

The unique capacity of dendritic cells (DCs) to induce both immunity and tolerance has attracted broad scientific interest. Many approaches have been attempted to generate suppressive DCs, among them the expression of a tryptophan catabolising enzyme called Indoleamine-2,3-Dioxygenase (IDO). We have recently reported data on IDO expressing human DCs contradicting reports of other groups, but here we show that enhanced IDO metabolism can exclusively confer immunosuppressive capacities on IL3-derived DCs.

DCs were generated from plastic-adherent monocytes in the presence of IL-4 and IL-3 (IL-3 DCs) or IL-4 and GM-CSF (GM-CSF DCs), matured using a standard cytokine cocktail and in some experiments stimulated with IFN- $\gamma$ to produce IDO. These cells were either used as stimulators in allogeneic mixed lymphocyte reactions (MLRs) or peptide-pulsed for analysing their priming and recall potential for peptide-specific T-cell responses.

Despite expressing IDO, both mature GM-CSF and IL-3DCs exerted powerful allogeneic T-cell stimulatory capacities. Additional treatment with IFN- $\gamma$ significantly upregulated IDO and thus exclusively rendered IL-3 DCs suppressive for allogeneic T-cell stimulation. This was observed for both, allogeneic $\mathrm{CD} 4+$ and $\mathrm{CD} 8+\mathrm{T}$-cell responses in contrast to GMCSF DCs which were strong stimulators. In summary, high IDO-expressing IL-3 DCs are a promising tool for dampening alloreactive $\mathrm{CD} 4+$ and $\mathrm{CD} 8+\mathrm{T}$-cell responses. Their peptide-specific stimulatory potential is currently under investigation.

\section{P074 \\ Restoring production of IL-12 family cytokines from dendritic cells altered by tumour exposure}

\section{A. Mulcahy, A. M. Jackson, X. Zhu, P. M. Patel; University of Nottingham, Nottingham, $U K$}

A key barrier in successful immune therapy for cancer is tumour induced immune-suppression. Regardless of the type of immunotherapy applied dendritic cells play a pivotal role in immune activation and several reports have demonstrated in vivo inhibition of DC IL-12 production and T-cell priming, however there is no data on the effect of tumours on IL-23 and IL-27. The IL-12 family of cytokines play important roles in anti-cancer immune responses via the priming of naïve $\mathrm{T}_{\mathrm{H}^{-1}}$ and increasing effector cytotoxicity and both IL-23 and IL-27 are purported to aid tumour clearance. The mechanism of suppressing IL-12 family for the purpose of immune escape is not well understood and restoring the IL-12 production of tumour exposed DCs would be an important step in the progress of immunotherapy and provide insight into the mechanisms of tumour DC subversion. We have shown that from TLR ligand stimulated monocyte-derived DCs melanoma exposure reduces IL-12 and IL-27 by $50 \%$ but not IL-23 and that the relative balance of these cytokines is shifted. These DCs have reduced $\mathrm{T}_{\mathrm{H}^{-}} 1$ stimulatory capacity despite no change in $\mathrm{MHC}$ and co-stimulatory molecule expression implying cytokine changes are responsible. Examining signalling molecules involved in both TLR signalling and IL-12 regulation revealed melanoma induced phosphoryla- 
tion of the mitogen-activated protein-kinase (MAPK) ERK. Inhibition of ERK restores IL-12 and $\mathrm{T}_{\mathrm{H}^{-1}}$ stimulation in melanoma exposed DCs and represents a potential target for future immunotherapy research.

\section{P075}

Generation of functionally competent dendritic cells from elutriated monocytes using various combinations of maturation factors

\author{
H. Kim $^{1}, H . \mathrm{Kim}^{2}$, S. $\mathrm{Kim}^{3}$, Y. Choi ${ }^{1}$; \\ ${ }^{1}$ Cell Therapy Center, Yonsei University College of Medicine, \\ Seoul, Republic of Korea, ${ }^{2}$ Department of Laboratoy Medicine, \\ Yonsei University College of Medicine, Seoul, Republic of Korea, \\ ${ }^{3}$ Department of Laboratory Medicine, Yonsei University College of \\ Medicine, Seoul, Republic of Korea
}

As dendritic cell (DC) vaccination is now being testing in trials with larger patient pools, clinical studies require sufficient quantities of well-characterized, highly immunogenic DC produced according to GMP guidelines. Although several maturation factors for improving DC yields have been evaluated in clinical trials, a detailed comparison of the functional potential of DCs from elutriated monocytes in response to different combinations of maturation factors has yet to be reported. Here, we compared the functional capacity of DCs in response to all possible pairwise combinations of 6 different classes of stimuli: (1) TNF- $\alpha$, (2) CD40L, (3) IFN- $\gamma$, (4) peptidoglycan, (5) poly I:C, and (6) monophosphoryl lipid A. Significant differences in the final functional capacities were found in the resultant DC lots in phenotype, cytokine secretion, and T-cell stimulation. DCs secrete significant amounts of IL-12 in presence of poly I:C or MPL, and mDC cultured with IFN- $\gamma+$ PGN produced the highest amounts of IL-12. IL-10 secretion was greatly induced in the presence of TLR ligands alone or in combination with other factors. While both types of mDCs were equivalent in their phenotype and capacity to stimulate allogeneic T-cell proliferation, IFN- $\gamma+$ PGN induced significantly less IL-10 and more IL-12 secretion than that of CD40L + PGN. Thus, DCs generated from elutriated monocytes efficiently stimulate T-cell proliferation and IL-12 secretion with Th1 differentiation capacity after maturation in the presence of PGN and IFN- $\gamma$. These differences are very useful for establishing DC maturation protocols for clinical applications.

\section{P076 \\ Functional dichotomy of plasmacytoid dendritic cells: specific activation of $T$-cells versus production of type I interferon}

\section{P. S. Jaehn, J. Schmitz, A. Dzionek; Miltenyi Biotec, Bergisch Gladbach, Germany}

Plasmacytoid dendritic cells (PDC) are recruited to tumor tissue and subvert tumor immunity by inducing $\mathrm{CD} 8+$ regulatory $\mathrm{T}$ cells. On the other hand, PDC can prime antigen-specific CD8+ and $\mathrm{CD} 4+\mathrm{T}$ cell responses both in vitro and in vivo. This indicates that PDC play a role in the induction and maintenance of $\mathrm{T}$ cell tolerance as well as in the induction of $\mathrm{T}$ cell immunity. To elucidate under which conditions PDC support $\mathrm{T}$ cell tolerance or immunity has spurred the current study. To this end, we designed a $\mathrm{T}$ cell restimulation system in which CMV antigen is specifically targeted via CD303 (BDCA-2) to PDC.
Subsequently, PDC are used to restimulate autologous CD4+ effector-memory $\mathrm{T}$ helper cells from $\mathrm{CMV}$ seropositive donors. We found a remarkable functional dichotomy of PDC and a novel pleiotropic effect of IFN-I: dependent on the type of stimulus, PDC produce either IFN-I, which induces the expression of the coinhibitory molecule PD-1L in an autocrine fashion or PDC process and present antigen to $\mathrm{T}$ cells leading to $\mathrm{T}$ cell activation. Both functions appear to be induced in a mutually exclusive fashion. Interestingly, IFN-I itself induces PD-1L expression in freshly isolated but not in mature PDC. Currently we investigate the outcome of IFN-I stimulation on immature and mature PDC regarding their capacity to induce $T$ cell activation or suppression. In vivo targeting of antigens to PDC via CD303 combined with appropriate PDC-stimulation may allow stimulation of specific anti-tumor $\mathrm{T}$ cell immunity or induction of tolerance.

\section{P077}

In vitro and in vivo inhibition of dendritic cell activation by MCS-18, a novel natural product isolated from Helleborus purpurascens

\author{
B. Horstmann ${ }^{1}$, E. Zinser ${ }^{1}$, N. Turza ${ }^{l}$, F. Kerek ${ }^{2}$, \\ A. Steinkasserer ${ }^{1}$; \\ ${ }^{1}$ University, Erlangen, Germany, ${ }^{2}$ DoNatur GmbH, \\ Martinsried, Germany
}

MCS-18, a novel natural product isolated from Helleborus purpurascens, is able to inhibit the expression of typical molecules of mature DC such as CD80, CD86, and especially of CD83 subsequently leading to a clear and dose dependent inhibition of the DCmediated T cell-stimulation. MCS-18 impeded the formation of the typical DC/T cell clusters. Interestingly, MCS-18 also inhibited CCR7 expression on DC which subsequently led to a dose dependent block of the CCL19-mediated DC-migration. MCS-18 inhibited the anti-CD3/anti-CD28 mediated $\mathrm{T}$ cell-stimulation. Strikingly, MCS-18 also strongly reduced the paralysis associated with the experimental autoimmune encephalomyelitis (EAE) which is a murine model for human multiple sclerosis in a prophylactic as well as in a "real" therapeutic setting. Even when the EAE was induced for a second time MCS-18-treated animals were still protected, suggesting that MCS-18 induces a long lasting suppressive effect. In addition, and very important for the potential practical application in humans, MCS-18 was also active when administered orally. MCS-18-treatment almost completely reduced leukocyte infiltration in the brain and in the spinal cord. In conclusion, using in vitro as well in vivo assays we were able to show that MCS-18 exerts a strong immunosuppressive activity with highly interesting potential for the therapy of diseases characterized by a pathologically over-activated immune system.

P078

Intracellular expression of CD40L after adenoviral transduction in combination with IFN-gamma treatment generates human dendritic cells that both secrete IL-12 and have migratory functions
I. Knippertz ${ }^{1}$, A. Hesse ${ }^{1}$, E. Kaempgen ${ }^{1}$, G. Schuler ${ }^{1}$, A. Steinkasserer ${ }^{1}$, D. M. Nettelbeck ${ }^{1,2}$ 
${ }^{1}$ Department of Dermatology, University Hospital Erlangen, Erlangen, Germany, ${ }^{2}$ Helmholtz-University Group Oncolytic Adenoviruses at DKFZ and Department of Dermatology, German Cancer Research Center and Heidelberg University Hospital Heidelberg, Heidelberg, Germany

In Dendritic cell (DC)-based vaccination trials often monocytederived DCs (moDCs) matured with a cocktail composed of IL-1B, TNF-alpha, IL-6 and $\mathrm{PGE}_{2}$ have been used. It has been shown that $\mathrm{PGE}_{2}$ is indispensable for DC-migration but it is also known to inhibit IL-12p70 expression. Moreover other labs reported that these two essential functions of DCs are frequently not linked. Here we describe a new and interesting method to overcome this problem and to generate mature moDC capable of both migrating and producing IL-12p70. This could be achieved by a combined treatment with recombinant human interferon gamma (rh-IFNgamma) and adenoviral gene transfer of the trimeric CD40L. Immature moDCs were infected with an adenovirus serotype 5 coding for the trimeric human CD40L (Ad5hCD40L) in an autologous monocyte-conditioned medium supplemented with GM-CSF, IL-4, IL-1B, TNF-alpha, IL-6 and PGE 2 . Afterwards $(1.5 \mathrm{~h}) \mathrm{rh}$-IFN-gamma was added to the infected cells. $24 \mathrm{~h}$ later cells were checked for their migratory capacity, their IL-12 production as well as their CD40L expression and maturation status. Interestingly, moDCs infected with Ad5hCD40L and subsequently treated with rh-IFN-gamma fully matured, showed a high IL12 p70 secretion $(\sim 700 \mathrm{pg} / \mathrm{ml})$ as well as high migratory capacity. Even when the migrated cells were cultured for an additional time period of $24 \mathrm{~h}$ they were still able to secrete notable amounts of IL12 p70 $(\sim 160 \mathrm{pg} / \mathrm{ml})$. In summary, we could generate mature DCs which are able to both (i) migrate and (ii) secrete high levels of IL12 p70 providing a potentially new therapeutic tool for DC-based vaccination trials.

\section{P079 \\ Differential activation of human dendritic cells by lipopolysaccharide, double-stranded RNA, proinflammatory cytokines and human heat-shock protein 70}

\section{Madrigal-Estebas ${ }^{1,2}$, D. McNally ${ }^{2}$, E. Ruiz-Zamora ${ }^{2}$, D. G. Doherty ${ }^{l}$, M. A. Fares $^{2}$; \\ ${ }^{1}$ Institute of Immunology, Biology Dept, National University of Ireland Maynooth, Maynooth, Ireland, ${ }^{2}$ Genetics Department, Trinity College Dublin, Dublin, Ireland}

Dendritic cells (DCs) can be induced to mature using various stimuli. HSP70 has been shown to activate innate and adaptive immune responses, but some reports suggest that this effect may be due to contaminating endotoxin. We analysed human monocyte-derived DC maturation and DC-mediated T-cell activation in response to human heat shock protein-70 (hHSP70), E. coli HSP70 (DnaK), bacterial lipopolysaccharide (LPS), doublestranded RNA (poly I:C), and a proinflammatory cytokine cocktail consisting of IL- $1 \beta$, IL- 6 and TNF- $\alpha$, in the absence or presence of polymyxin B (PMB). All effects caused by DnaK were abrogated by PMB, indicating LPS contamination. However, hHSP70, even with PMB, enhanced surface HLA-DR, CD80, CD86 and CD54 expression similarly to LPS, poly I:C and the cytokine cocktail. In contrast to LPS, neither hHSP70, poly I:C, nor the cytokine cocktail induced IL-12 nor IL-10 release by DC. In contrast to LPS, poly I:C and the cytokine cocktail, hHSP70 did not upregulate CD40 expression by DC. However, hHSP70 or
LPS were more potent inducers of CD83 expression than poly I:C and the cytokines. hHSP70-matured DC did not induce proliferation, IFN- $\gamma$ or IL-10 secretion by allogeneic T cells, but these functions were induced by DC matured with LPS or poly I:C. In summary, hHSP70 can induce maturation of DC into cells that are phenotypically distinct from DC matured by other stimuli and which in our system do not induce $\mathrm{T}$ cell activation. These data suggest a role for hHSP70 in immune modulation at the level of the DC.

\section{P080}

The influence of tumour cells on the crosstalk between activated or resting natural killer and dendritic cell

\section{G. C. Sim, D. Schadendorf, A. Paschen; DKFZ, Mannheim, Germany}

The outcome of the bidirectional cross-talk of natural killer cells (NK)-monocyte-derived dendritic cells (moDCs) is dependent on the different activation stage of NK cells and moDCs and may be regulated by the tumour microenvironment. In this study, we investigated how melanoma cells, Mel249 in the presence or absence of IFN- $\alpha$ modulate the phenotype and cytokine secretion of immature moDCs co-cultured with resting or activated NK cells in vitro. Our results indicate that activated NK cells are more efficient than resting NK cells in inducing an up-regulation of CD86, CD80, CD40, CD83 and HLA-DR on moDCs. Resting NK cells are capable of inducing comparable CD80 and CD86 expression levels only in the presence of IFN- $\alpha$ and Mel249. Besides, Mel249 enhances the secretion of IL-12p70 by moDCs co-cultured with activated NK cells post-CD40L ligation. Interestingly, the presence of IFN- $\alpha$ prevents the high IL12p70 secretion. moDCs co-incubated with resting NK cells secrete lower levels of IL-12p70. In contrast, the IFN- $\alpha$ signal is important in increasing IL-12p70 secretion when moDCs were co-cultured with resting NK cells in the presence of Mel249. Thus, in the presence of the signals provided by Mel249, activated NK cells-mediated moDCs maturation is predicted to be superior to resting NK cells-mediated moDCs maturation in eliciting a Th1 type immune response. The optimal polarisation of moDCs by NK cells of different activation stage is influenced by the balance of signals provided by tumour cells and also by IFN- $\alpha$.

\section{P081}

\section{Modulation of viability and maturation of human monocyte-derived dentritic cells by oncolytic adenoviruses}

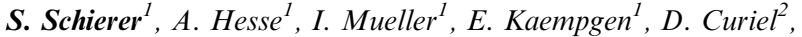 G. Schuler ${ }^{1}$, A. Steinkasserer ${ }^{1}$, D. Nettelbeck ${ }^{1,3,4}$; \\ ${ }^{1}$ Department of Dermatology, University Hospital Erlangen, Germany, ${ }^{2}$ Human Gene Therapy and the Gene Therapy Center, Birmingham, AL, USA, ${ }^{3}$ Helmholtz-University Group Oncolytic Adenoviruses at DKFZ, German Cancer Research Center Heidel- berg, Germany, ${ }^{4}$ Department of Dermatology, University Hospital Heidelberg, Germany}

Adenoviral oncolysis is a promising new modality for treatment of cancer based on selective viral replication in tumor cells. However, tumor cell killing by adenoviral oncolysis needs to be improved in 
order to achieve therapeutic benefit in the clinic. Towards this end, the activation of anti-tumor immunity by adenoviral oncolysis might constitute a potent mechanism for systemic killing of uninfected tumor cells, thereby effectively complementing direct tumor cell killing by the virus. Knowledge of anti-tumor immune induction by adenoviral oncolysis, however, is lacking mostly due to species-specificity of adenovirus replication, which hampers studies of human oncolytic adenoviruses in animals. We suggest the analysis of interactions of oncolytic adenoviruses with human immune cells as rational basis for the implementation of adenoviral oncolysis-induced anti-tumor immune activation. The goal of this study was to investigate how oncolytic adenoviruses affect human dendritic cells (DCs), the key regulators of innate and adoptive immunity that are widely investigated as tumor vaccines. We report that melanoma-directed oncolytic adenoviruses, like replication-deficient adenoviruses but unlike adenoviruses with unrestricted replication potential, are not toxic to monocyte-derived immature DCs and do not block DC maturation by external stimuli. Of note, this is in contrast to reports for other viruses/ viral vectors and represents a pre-requisite for anti-tumor immune activation by adenoviral oncolysis. Furthermore, we show that these oncolytic adenoviruses alone do not or only partially induce DC maturation. Thus additional signals are required for optimal immune activation. These could be delivered, for example, by inserting immunoregulatory transgenes into the oncolytic adenovirus genome.

\section{Oral Presentations (17 July 2007)}

\section{P082}

\section{Dendritic cell vaccination with heteroclitic polypeptide hormone leads to diminished tumor outgrowth in neuroendocrine cancer}

\author{
M. Wuttke, C. Papewalis, Y. Meyer, C. Kessler, B. Jacobs, \\ W. A. Scherbaum, M. Schott; \\ Department of Endocrinology, Duesseldorf, Germany
}

Neuroendocrine cancers are characterized by low sensitivity to chemotherapy or radiation and frequently by a devastating prognosis. Here, we tested whether the tumor-specific polypeptide hormone (calcitonin) for medullary thyroid carcinoma (MTC) may serve as target molecule for immunotherapy in a transgenic mouse model for MTC (Ret/Cal mice). Ret/Cal mice displaying the same mutation within the Ret-Protooncogene as most patients with hereditary MTC and, therefore, develop MTCs with onehundret percent tumor prevalence.

$30 \mathrm{Ret} / \mathrm{Cal}$ mice were monthly immunized with calcitoninpulsed dendritic cells (DCs). After a follow-up of 6 months, mice were sacrificed for further analyses. Paratumoreous lymph nodes from control mice did not show any abnormalities although amino acid-modified (heteroclitic) calcitonin-treated mice did. For enumerating of epitope-specific CTLs these lymph nodes $(\mathrm{LN})$ together with spleens were extracted and tetramer analyses were performed. About $8 \%$ of CTLs from cervical LN were detected by at least one of three tetramers tested. In parallel, MTCs of calcitonin-treated mice showed in some cases strong CTL infiltrations. To test whether these cells may lyse MTC cells splenic interferon- $\gamma$ secreting CTLs were isolated for in vitro cytotoxicity assays. On the basis of ${ }^{51} \mathrm{Cr}$ release up to $41 \%$ cytotoxic activity was seen. Mostimportant, we examined the tumor outgrowth by measuring tumor sizes in anti-calcitonin stained serial cryostat sections of the thyroid glands.
Strikingly, calcitonin-immunized mice revealed a largely reduced tumor outgrowth compared to control mice $(-76 \%)$. These results have a major impact as they describe that polypeptide hormones may serve as target molecule for immunotherapy in endocrine malignancies.

\section{P083 \\ Immunotherapy for relapsed high grade glioma: analysis of three cohorts of the cohort comparison trial HGG-IMMUNO-2003}

S. W. Van Gool ${ }^{1}$, S. Rutkowski ${ }^{2}$, E. Kaempgen ${ }^{3}$, J. Wolff ${ }^{4}$, S. Wagner ${ }^{5}$, F. Van Calenbergh ${ }^{1}$, C. Kramm ${ }^{6}$,

S. De Vleeschouwer ${ }^{1}$;

${ }^{1}$ Catholic University of Leuven, Leuven, Belgium, ${ }^{2}$ University of Wuerzburg, Wuerzburg, Germany, ${ }^{3}$ University of Erlangen, Erlangen, Germany, ${ }^{4}$ MDAnderson Cancer Center, Houston, TX, USA, ${ }^{5}$ University of Regensburg, Regensburg, Germany,

${ }^{6}$ University of Halle, Halle, Germany

Purpose: The prognosis of patients with HGG is dismal. We investigate the therapeutic role of vaccination with mature DC loaded with tumor lysates derived from resected tumor at time of relapse.

Methods: DC were injected intradermally according to three different vaccination schedules.

Results: 87 patients entered these cohorts. The median age at time of relapse of HGG was 42 year (3-77 years). Nine patients dropped out immediately due to tumor progression. Six patients with concomittant chemotherapy were excluded for the as-treated analysis. The median PFS and OS of 72 patients was 4 and $11 \mathrm{~m}$ (2-year OS $=19 \%)$. For the subgroup of relapsed GBM, median age was 45 years (7-77). The median PFS and OS of the total group was 3 respectively $10 \mathrm{~m}$ (2-year OS $=13 \%$ ). Age, extent of resection and pathology were independent risk factors. By stepwise shortening the interval between the vaccination from cohort A to C, the PFS of adults with relapsed GBM significantly improved. All patients received their vaccinations in an ambulatory setting. There were no adverse events except in one relapsed patient with gross tumoral disease prior to vaccination, who developed repetitive vaccine-related peritumoral edema.

Conclusion: Immunotherapy for patients with HGG is feasible without major adverse events. Especially in young patients DC vaccination induces obvious tumor control even at time of relapse. The induction of PFS $>5$ year in some patients provides a level $1 \mathrm{c}$ medical evidence of efficacy of $\mathrm{DC}$ vaccination as treatment modality against HGG.

\section{P084}

Abstract unavailable

\section{P085}

Randomized phase III trial of adjuvant active specific immunization with Newcastle Disease Virus modified tumor cells in metastasized colorectal cancer

\author{
T. Schulze ${ }^{1}$, W. Kemmner ${ }^{1}, J$. Weitz $^{2}$, K. Wernecke ${ }^{3}$, \\ V. Schirrmacher ${ }^{4}$, P. M. Schlag ${ }^{1}$;
}


${ }^{1}$ Department of Surgery and Surgical Oncology, Charité Campus Berlin-Buch, Universitätsmedizin Berlin, Berlin, Germany, ${ }^{2}$ Department of Surgery, University of Heidelberg, Heidelberg, Germany, ${ }^{3}$ Insitut of Medical Biometry, Charité, Universitätsmedizin Berlin, Berlin, Germany, ${ }^{4}$ Division of Cellular Immunology, German Cancer Research Center, Heidelberg, Germany

Purpose: Metastatic disease is a major cause of mortality in colorectal cancer patients. Even after complete resection of isolated liver metastases, recurrence develops in the majority of patients. Therefore, development of strategies to prevent recurrent liver metastases is of major clinical importance. The present prospectively randomised phase III trial investigates the efficiency of active specific immunotherapy (ASI) after liver resection for hepatic metastases of colorectal cancer.

Methods: Patients with histologically confirmed liver metastases from colorectal cancer were randomised to the vaccination or control group. After complete resection of liver metastases, patients randomised to the vaccination group received six doses of NDV infected autologous tumour cell vaccine (ATV-NDV). The primary end-point was overall survival, secondary end-points were disease-free survival and metastases-free survival.

Results: Fifty-one patients were enrolled in the study with 50 patients available for analysis. The follow-up period was $116.1 \pm 23.8$ month in the vaccination arm and $112.4 \pm 18.5$ month in the control group. In the total patient group, no differences in the primary and secondary end-points were detected. Most interestingly, subgroup analysis revealed a significant advantage for vaccinated colon cancer patients with respect to overall survival (Hazard ratio: 3.3; 95\%-Confidence interval (CI): $1.0-10.4 ; P=0.042$ ) and metastases-free survival (Hazard ratio: 2.7; 95\%-CI: $1.0-7.4 ; P=0.047$ ) in the intention-to-treat analysis. Conclusion: ASI in unselected colorectal cancer patients was not effective for prevention of recurrent metastatic disease. However, in colon cancer patients, ASI appears to be beneficial prolonging overall and metastases-free survival.

\section{P086}

A randomized, controlled, phase II study with an MVA-Nef vaccine in HIV-1 infected patients followed by structured treatment interruption (STI)

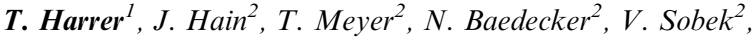 \\ M. Baeuerle ${ }^{1}$, H. Jaeger ${ }^{3}$, M. Helm $^{4}$, L. Schneider ${ }^{5}$, D. Gorriahn ${ }^{6}$, \\ E. G. Harrer ${ }^{1}$; \\ ${ }^{I}$ Department of Medicine III, University Hospital Erlangen, \\ Erlangen, Germany, ${ }^{2}$ Bavarian Nordic GmbH, Martinsried, \\ Germany, ${ }^{3}$ Practice Dr. Jaeger, Munich, Germany, ${ }^{4}$ Practice Dr. \\ Helm, Nuremberg, Germany, ${ }^{5}$ Practice Dr. Schneider, Fürth, \\ Germany, ${ }^{6}$ Practice Dr. Gorriahn, Munich, Germany
}

Objective: To assess the immunogenicity and safety of an MVA vector expressing HIV-1-LAI nef in HIV-1 infected patients on HAART with CD4 counts $\geq 250 / \mu 1$.

Methods: In this single blind, controlled, randomized, multicenter phase-II-trial 77 HIV-1 infected patients received three s.c. vaccinations of either 1E8 MVA-BN, 1E8 MVA-nef or 5E8 MVA-nef ( $n=25,26$ and 26) at week 0,8 and 16. 60/77 subjects were preimmune to vaccinia due to prior smallpox vaccination. Patients that had received all vaccinations and remained stable on HAART with HIV-1-RNA levels $<50$ copies/ml were offered a
Structured Treatment Interruption (STI) at week 20 and were followed up until week 52 .

Results: MVA-Nef proved to be safe and well tolerated. All 77 subjects received three vaccinations. Thirty-seven subjects interrupted HAART 4 weeks following the third vaccination $(9: 13: 15$ for 1E8 MVA-BN, 1E8 MVA-nef, 5E8 MVA-nef,respectively). During the 32 week post STI period 12 of 37 subjects restarted antiretroviral therapy, mostly due to increasing VL; these patients came back to contolled VL after re-start of HAART. Data collected from the 25 patients followed up until week 52 indicate a significant better control of the VL in the group vaccinated with 5E8 MVA-Nef compared to the MVA-BN vaccinated control group. All patients developed a strong MVA-specific humoral immune response.

Conclusions: The results of this study confirm the promising data of a previous Phase I trial which indicated a trend in controlling VL during STI after therapeutic immunisation with MVA-nef (Harrer et al. in Antivir Ther 2005).

\section{Clinical Trials}

\section{P087} Immunotherapy for malignant melanoma using
recombinant NY-ESO-1 protein and Toll-like
receptor 7 agonist imiquimod as vaccine adjuvant

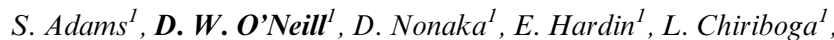

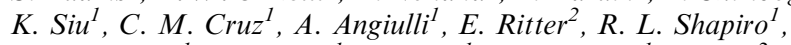
R. S. Berman ${ }^{1}$, N. Berner ${ }^{1}$, Y. Shao', O. Manches ${ }^{1}$, L. Pan ${ }^{2}$, E. Hoffman ${ }^{2}$, S. Gnjatic ${ }^{2}$, L. Old ${ }^{2}$, A. C. Pavlick ${ }^{1}$, N. Bhardwaj ${ }^{1}$; ${ }^{1}$ New York University School of Medicine, New York, NY, USA,

${ }^{2}$ Ludwig Institute for Cancer Research, New York, NY, USA

$\mathrm{T}$ cell-mediated immunity to microbes and to cancer is triggered by activation of dendritic cells (DCs) via Toll-like receptors (TLRs). Here we evaluated the safety and adjuvant activity of imiquimod, a TLR7 agonist, in a vaccine against cancer/testis antigen NY-ESO-1 in patients with malignant melanoma. We assessed the ability of topical imiquimod to recruit and activate DCs in the skin and to generate humoral and $\mathrm{T}$ cell-mediated immunity to NY-ESO-1. Nine patients with resected stage IIB-IIIC melanoma received four vaccinations with recombinant NY-ESO-1 protein, injected intradermally into skin treated with imiquimod cream. Peripheral blood samples were evaluated for evidence of vaccine-induced immunity, and skin biopsies were assessed for imiquimod-induced in situ immunomodulatory effects. The regimen was very well-tolerated with only mild local reactions and constitutional symptoms. It induced measurable NY-ESO-1-specific CD4 $+\mathrm{T}$ cell responses in six of eight evaluable patients. CD8 $+\mathrm{T}$ cell responses were not detected. Humoral immunity was manifested by the development of NY-ESO-1specific antibodies in four of nine patients. Imiquimod induced dermal mononuclear cell infiltrates in all patients composed primarily of T cells, monocytes, macrophages, myeloid DCs and NK cells, and to a lesser extent plasmacytoid DCs. DC activation was evident. This study demonstrates the feasibility and safety of topical application of a TLR agonist as a vaccine adjuvant in human subjects. The use of imiquimod for this purpose shows promise, although parameters such as formulation, dose and timing relative to antigen exposure need to be optimized. 


\section{P088}

Vaccination of acute myeloid leukemia (AML) patients with clinical grade mature dendritic cells (DC) electrotransfected with messenger RNA encoding the Wilms' tumor protein WT1: a phase I trial

A. Van Driessche ${ }^{l}$, A. Van de Velde ${ }^{I}$, G. Nijs ${ }^{1}$, A. P. Gadisseur ${ }^{1}$, W. A. Schroyens ${ }^{1}$, I. J. De Vries $^{2}$, Z. N. Berneman ${ }^{1}$,

V. F. Van Tendeloo ${ }^{1}$;

${ }^{1}$ Antwerp University Hospital, Edegem, Belgium,

${ }^{2}$ Radboud University Nijmegen, Nijmegen, The Netherlands

WT1 is a promising target for immunotherapeutic interventions of WT1-overexpressing tumors such as AML. We conducted a phase I vaccination trial in AML remission patients to evaluate a clinical grade DC vaccine loaded with WT1-encoding mRNA.

Five patients with AML in remission were included, underwent a standard apheresis and received four intradermal DC vaccinations. Mature DC (mDC) were generated from CD14-isolated monocytes in serumfree medium supplemented with $1 \%$ human serum with GM-CSF and IL-4 followed by maturation with TNF$\alpha$ and prostaglandin E2 (and KLH as helper antigen). mDC were transfected with GMP-grade mRNA encoding WT1 and patients received four biweekly vaccines and a delayed-type hypersensitivity (DTH) test was done 2 weeks after the fourth vaccination. Flow cytometry confirmed DC viability and mature phenotype. WT1 tumor marker RNA levels in peripheral blood were monitored by quantitative RT-PCR.

Vaccine production was successful for all patients. Release criteria for viability $(>70 \%)$, sterility and phenotype (CD80 + CD86 + CD83 + HLA/DR + CCR7 + CD14-) were fulfilled for all vaccines produced. DC injections were well tolerated and no serious adverse effects were observed. DTH reactions showed vaccine-specific cellular immune response, especially to $\mathrm{KLH}$, in all patients. A tendency for decrease in WT1 RNA levels during the vaccination cycle was detectable in $4 / 5$ patients, and one patient remains disease-free although follow-up is relatively short. Immunomonitoring of WT1-specific T cell responses will be performed in patients evaluable by $\mathrm{T}$ cell assays.

We conclude that immunotherapy with the described DC vaccine is feasible and safe, and that the vaccine can elicit antivaccine $\mathrm{T}$-cell responses in vivo.

\section{P089}

\section{Vaccination with MVA-Nef is immunogenic} and safe in HIV-1 negative volunteers

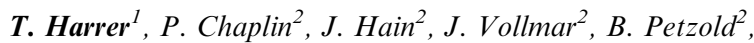
A. Handley ${ }^{2}$, S. Bergmann ${ }^{1}$, K. Eismann ${ }^{l}$, B. Spriewald ${ }^{l}$, D. Willbold ${ }^{3}$, E. G. Harrer ${ }^{1}$;

${ }^{I}$ Department of Medicine III, Erlangen, Germany,

${ }^{2}$ Bavarian Nordic GmbH, Martinsried, Germany,

${ }^{3}$ Heinrich-Heine-University, Juelich, Germany

Objective: To investigate the immunogenicity and safety of an MVA vector expressing the HIV-1-LAI Nef gene (MVA-nef), we performed a phase-I-study in healthy volunteers.

Methods: 14 HIV-negative subjects were immunized sc. with MVA-nef at week 0, 4 and 16. 10/14 subjects were vaccinia naive. Nef- and MVA-specific T-cells were monitored by g-IFN ELISPOT. A positive ELISPOT response was defined as $\geq 50 \mathrm{SFU} / 10^{6}$
PBMC after subtraction of a negative control. In additional stimulation assays thawed PBMC samples, frozen at the various time points of the study, were stimulated by HLA-matched peptide pools and outgrowing cells were analyzed after 1-2 weeks for recognition of the corresponding peptides.

Results: MVA-nef was well tolerated with injection site reactions in all subjects and mild systemic side effects in 10/14 subjects. All subjects developed a strong MVA-specific antibody response after the first vaccination and MVA-specific T-cells at week 18. Nefspecific T-cells were detected in freshly isolated PBMC of $7 / 14$ subjects (Nef-specific CTL in 6 subjects, Nef-spec. CD4-cells in one subjects and Nef-protein spec.T-cells in three subjects). ELISPOT analysis of peptide stimulated thawed PBMC detected induction of Nef-specific CTL during the vaccination period in additional two subjects. Nef-specific antibodies could be induced in two subjects.

Conclusions: MVA-nef was safe and immunogenic in uninfected subjects and induced a Nef-specific-T-cell response in 9/14 (64\%) of healthy volunteers, whereas Nef-specific antibodies could be detected only in $14 \%$. A strong MVA-specific T-cell and antibody response developed in all subjects.

\section{P090}

A Phase I Clinical Trial of a BDCA-1 ${ }^{+}\left(\mathrm{CD1c}^{+}\right)$ Blood Dendritic Cell Preparation Loaded With Control and Tumour-Associated Antigens, for the Immune Therapy of Metastatic Refractory Prostate Cancer

R. L. Prue, T. Rossetti, F. Vari, H. Tong, G. Crosbie, C. Tracey, H. Goossen, P. Swindle, D. N. J. Hart;

Mater Medical Research Institute, Brisbane, Australia

In vitro loaded dendritic cells (DC) are a promising new treatment for cancer patients. Blood DC (BDC) preparations are an attractive alternative to the commonly used monocyte-derived DC in terms of performance, logistics, cost and regulatory issues. Our pre-clinical studies have shown that isolated $\mathrm{CD}^{+} \mathrm{c}^{+} \mathrm{BDC}$ can induce MHC class I cytotoxic T lymphocyte responses to HLAA*0201 restricted tumour associated antigens and retain functional capacity following cyropreservation (Prostate 66:180, 2006).

We are conducting a Phase I clinical trial of $\mathrm{CD} \mathrm{c}^{+} \mathrm{BDC}$ pulsed with TAA, (PSA-3, PSMA-2, PAP-5), and control antigens influenza matrix protein peptide and Keyhole Limpet Hemocyaninin for the immunotherapy of metastatic hormone refractory prostate cancer. Patients receive 3 i.d. or i.v. injections at monthly intervals. The initial vaccine is administrated fresh and subsequent vaccines following cryopreservation. To date six patients have completed the immunotherapy protocol.

BDC are isolated using the CliniMACS ${ }^{\circledR} \mathrm{CDlc}^{+}\left(\mathrm{BDCA}_{-1}{ }^{+}\right)$ cell selection system. From a starting leukapheresis product (Mean: WBC $13.8 \times 10^{9}, N=6$ ) we obtained a mean of $39.5 \times 10^{6}$ $(N=6)$ cells with a mean CD1c ${ }^{+}$BDC purity of $86 \%(N=6)$. Recovery following cryopreservation was $52 \%(N=12)$, with a mean purity and viability $(N=12)$ of 86 and $90 \%$, respectively. PSA level, disease bulk, BDC numbers, DTH response and MHC class II and I restricted $\mathrm{T}$ cell responses are being assessed, in addition to other immunological parameters.

To date it has proven feasible to produce the BDC vaccine from this patient group and it has been well tolerated, demonstrating the practicability of using immunoselected cryopresevered BDC for immunotherapy protocols. 


\section{P091}

\section{Phase I/II study of immunotherapy using autologous tumor lysate-pulsed dendritic cells in patients with metastatic renal cell carcinoma}

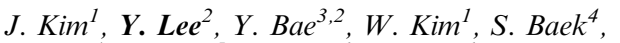 \\ H. Lee ${ }^{4}$, H. Doh ${ }^{5}$, C. Jeon ${ }^{6}$, C. Jung ${ }^{1}$; \\ ${ }^{1}$ Division of Hematology/Oncology, Department of Medicine, \\ Samsung Biomedical Research Institute, Samsung Medical Center, \\ Seoul, Republic of Korea, ${ }^{2}$ CreaGene Inc., Seongnam, Republic \\ of Korea, ${ }^{3}$ Department of Biological Science, Sungkyunkwan \\ University, Suwon, Republic of Korea, ${ }^{4}$ Samsung Biomedical \\ Research Institute, Samsung Medical Center, Seoul, Republic \\ of Korea, ${ }^{5}$ Research Institute, Dong-A Pharmaceutical Co., Ltd., \\ Giheung, Republic of Korea, ${ }^{6}$ CreaGene Inc., Seongnam-Si, \\ Gyunggi-do, Republic of Korea
}

This phase I/II study was conducted to evaluate the feasibility, safety, and efficacy of immunotherapy using autologous tumor lysate (TL)-pulsed dendritic cells (DCs) in patients with metastatic renal cell carcinoma (RCC). DCs were generated by culturing PBMCs in the presence of GM-CSF and IL-4 and were pulsed with autologous TL and keyhole limpet hemocyanin (KLH). Maturation of DCs was induced by a combined treatment of CD40 ligand, IFN- $\gamma$, and monocyte-conditioned medium. The patients were administered two cycles of DC vaccination, each of which comprised of four times injection subcutaneously at biweekly intervals.

Nine patients were included and well tolerated without severe side effects. One patient achieved an objective partial response (PR). Five patients showed stable disease (SD), and the remaining three had progressive disease (PD). With the median time to progression was 5.1 months and the median overall survival was 29 months. In the DTH test, six patients demonstrated a positive conversion to KLH and TL-pulsed DCs and the remaining three showed an augmented skin reaction after vaccination. In the KLH-induced lymphocyte proliferation assay, eight patients showed a marked enhancement of proliferative response, which tended to be stronger in patients with SD or PR than in patients with PD. The IFN- $\gamma$ ELISPOT assay was performed in two patients and had a strong response against KLH.

As a result, TL-pulsed DC-based immunotherapy in combination with nephrectomy affect the natural course of RCC and are associated with clinical benefits for patients with metastatic diseases

\section{P092}

\section{Phase I/II trial immunizing colorectal cancer patients with MelCancerVac ${ }^{\circledR}$ : issues on GMP vaccine production}

\author{
P. Kvistborg ${ }^{1}$, S. Burgdorf ${ }^{2}$, A. W. Pedersen ${ }^{1}$, A. Kirkin $^{1}$, \\ K. Dzhandzhugazyan ${ }^{1}, J$. Rosenberg ${ }^{2}$, M. H. Claesson ${ }^{3}$, \\ M. Zoccal; \\ ${ }^{1}$ Dandrit biotech, Copenhagen, Denmark, ${ }^{2}$ Department of surgical \\ gastroenterology, University hospital of Copenhagen Gentofte, \\ Copenhagen, Denmark, ${ }^{3}$ Department of Medical Anatomy, \\ University of Copenhagen, Copenhagen, Denmark
}

We performed a phase I/II trial testing the safety, toxicity (1) and clinical response of MelCancerVac ${ }^{\circledR}$, a vaccine consisting of autologous dendritic cells (DC) pulsed with allogenic tumor lysate. Patients received 10 doses of $3-5 \times 10^{6} \mathrm{DC}$, delivered intradermally.
The trial was conducted in our cGMP facilities and was the first Danish immunotherapy study fulfilling current EU GMP regulations. All vaccines produced were manufactured from peripheral blood $(200 \mathrm{ml})$ and we obtained vaccines in a range of 2-10 from one manufacturing round.

Vaccine production: Cell surface phenotype of the DC indicates that these DC were of intermediate maturity being HLA-D ${ }^{\text {high }}$, $\mathrm{CD}_{0} 0^{+}, \mathrm{CD}_{6} 6^{+}, \mathrm{CD} 83^{+/-}, \mathrm{CD} 14^{+/-}, \mathrm{CDla}^{+}$and $\mathrm{CCR} 7^{(+)}$.

Vaccine cytokine analysis: DC supernatants from four stable disease responders (SD) and four progressive disease non-resonders (PD) were analysed using a ten plex LUMINEX assay

Conclusion: Our GMP experiences from this study have made it possible to make QC guidelines. MelCancerVac ${ }^{\circledR}$ based on autologous DCs loaded with allogenic tumor lysate from a cell line known to have high MAGE expression. However, our study revealed that one of the SD patients had no MAGE tumor expression (1). Overall the study showed an impressively good performance of all patients throughout the study and stable Quality of life (QoL) data throughout and beyond the period of vaccination.

\section{Reference List}

1. Burgdorf SK, Fischer A, Claesson MH, Kirkin AF, Dzhandzhugazyan KN, Rosenberg J (2006) Vaccination with melanoma lysate-pulsed dendritic cells, of patients with advanced colorectal carcinoma: report from a phase I study. J Exp Clin Cancer Res $25: 201-206$

\section{P093}

Vaccination with autologous dendritic cells in bcr/abl + chronic myeloid leukemia: results of a clinical phase-I/II trial
J. Westermann ${ }^{1,2}$, J. Kopp ${ }^{2}$, A. van Lessen ${ }^{1}$, A. Hecker ${ }^{1}$, G. Baskaynak ${ }^{1}$, P. le Coutre ${ }^{1}$, K. Döhner ${ }^{3}$, H. Döhner ${ }^{3}$, B. Dörken ${ }^{1,2}$, A. Pezzutto $^{1,2}$;
${ }^{1}$ Charité-University Medicine Berlin, Campus Virchow-Klinikum, Berlin, Germany, ${ }^{2}$ Charité-University Medicine Berlin, Campus Berlin-Buch, Berlin, Germany, ${ }^{3}$ Department of Hematology and Oncology, University of Ulm, Ulm, Germany

In chronic myeloid leukemia (CML), DC and leukemic cells share a common progeny, leading to constitutive expression of putative tumor antigens such as bcr/abl in DC. T cells recognizing truly leukemia-specific antigens like bcr/abl or other leukemia-associated antigens such as proteinase- 3 and WT- 1 have been demonstrated by several groups.

In our clinical GMP study we used autologous DC as a vaccine in patients with chronic phase bcr/abl + CML, who had not achieved a major cytogenetic response after treatment with imatinib or $\alpha$-interferon.

10 patients were enrolled. DC were generated from peripheral blood monocytes in the presence of GM-CSF, IL- 4 and TNF- $\alpha$. In all patients, sufficient numbers of functional DC could be generated for subcutaneous vaccination. The mean value of bcr/abl-positive cells in the DC cultures was $33 \%$ (range 3-70\%) as determined by FISH analysis.

Vaccination was feasible and safe. An improvement of the cytogenetic/molecular response, as detected by FISH, was possibly related to vaccination in $4 / 10$ patients. In three of these patients, $T$ cells recognizing leukemia-associated antigens became detectable. All patients developed a strong DTH reaction, mostly after the third vaccination. The proliferative capacity of PBMC in response to autologous DC increased in all evaluable patients. 
We conclude that vaccination of CML patients after large scale GMP generation of bcr/abl + DC is feasible and safe. Leukemic $\mathrm{DC}$ are able to induce $\mathrm{T}$ cell responses in some patients.

DC vaccination might be particulary useful in CML as post remission therapy, i.e. after treatment with tyrosine kinase inhibitors

\section{P094}

\section{Immunological and clinical responses after vaccinations with RNA coding for tumour associated antigens in advanced RCC patients}

S. M. Schmidt ${ }^{1}$, M. Haentschel ${ }^{1}$, M. R. Mueller ${ }^{1}$, K. J. Weimer ${ }^{1}$, K. J. Weimer ${ }^{1}$, S. Wirths ${ }^{1}$, J. Wierecky ${ }^{2}, W$. Brugger ${ }^{3}$,

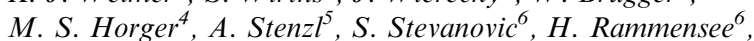
L. Kanz ${ }^{1}$, S. Pascolo ${ }^{6}$, P. Brossart ${ }^{1}$;

${ }^{1}$ Department of Hematology, Oncology, Rheumatology and Immunology, Tuebingen, Germany, ${ }^{2}$ Department of Hematology, Oncology, Hamburg, Germany, ${ }^{3}$ Department of Hematology, Oncology, Villingen-Schwenningen, Germany, ${ }^{4}$ Department of Radiology, Tuebingen, Germany, ${ }^{5}$ Department of Urology, Tuebingen, Germany, ${ }^{6}$ Department of Immunology, Tuebingen, Germany

We conducted a phase I/II trial to evaluate feasibility, safety and efficacy as well as immunological responses of a RNA based vaccination in patients with metastatic renal cell carcinoma (RCC). Vaccination was performed with mRNA encoding for tumour associated antigens (TAA) using two different treatment arms.

In vitro transcribed RNA was generated using plasmids coding for the TAA MUC1, CEA, Her-2/neu, telomerase, survivin and MAGE-1. Vaccinations were performed intradermally on days 1, 14, 28 and 42 in arm A and on days $0-3,7-10,28$ and 42 in an intensified arm B. Subsequently vaccinations were repeated monthly until tumour progression. On the day following RNA vaccination GM-CSF was applicated s.c.. Induction of vaccineinduced T-cell responses was monitored using IFN-g ELISPOT assays.

Thirty patients aged 36 to 79 years were enrolled, 14 in arm A and 16 in Arm B. Treatment was well tolerated with no severe side effects.

In five patients of arm A and four of arm B stable disease was achieved for at least 5 months, two showed temporary regression of metastases. Three patients were stable for over 1 year $(12,17$, and 18 months). Fifteen patients were still alive with disease 9 to 28 months after ending of the study.

In ongoing ELISPOT assays antigen-specific T-cell induction could be shown for several TAA in 7 of 9 analysed patients after 8 vaccinations.

This study demonstrates that vaccination with RNA coding for TAA can be effective in the treatment of RCC patients and induces clinical and immunological responses.

\section{P095 \\ Dendritic cell immunotherapy for advanced metastatic melanoma: interim report}

N. L. Wilson, A. Gravett, D. Fowler, A. Dalgleish; St Georges University of London, London, UK
Dendritic cell (DC) based therapy has been reported to induce marked clinical responses albeit infrequently in a number of tumour types. However, the number of different variables makes comparison difficult. We decided to incorporate those features which appear to be associated with an improved outcome in several studies.

Monocyte-derived DC cultured in GM-CSF and IL-4 were used. Antigens were provided by a lysate prepared from three allogeneic cell lines expressing at least 4 shared antigens (MAGE, MART-1, gp100, tyrosinase). The injection site was primed with imiquimod cream and half the injections were given intra-tumourally or -nodally and half the dose intradermally. Patients were then boosted with low dose IL-2 to expand primed killer T cells. The vaccine was given fortnightly for five administrations and monthly for the next four. Primary endpoints are the immune response, time to disease progression and survival. Secondary endpoints are tumour response as well as response to salvage treatment such as chemotherapy and radiotherapy.

Treatment has commenced for 30 patients, all of whom have measurable visceral disease. To date clinical responses have only been seen in small volume disease. Stable disease has been observed in previously progressive bulky disease. Notably, some patients report an improvement in quality of life and weight gain. Detailed analysis will be reported. A major issue in this study is the aggressive nature of stage IV disease, with three patients showing progressive disease within the period of enrolment and DC vaccine production. These patients were given chemotherapy as well as DCs.

\section{P096 \\ Vaccination with type-1 dendritic cells pulsed with multiple glioma-associated antigen peptides in combination with poly-ICLC in patients with recurrent malignant glioma}

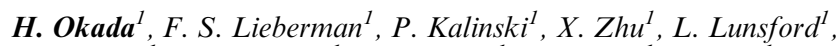

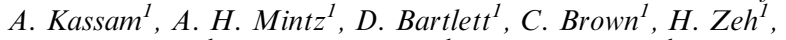

T. L. Whiteside ${ }^{l}$, L. H. Butterfield ${ }^{l}$, R. L. Hamilton ${ }^{1}$, A. M. Salazar ${ }^{2}$, I. F. Pollack ${ }^{1}$;

${ }^{1}$ University of Pittsburgh Cancer Institute, Pittsburgh, PA, USA,

${ }^{2}$ Oncovir, Inc., Washington, DC, USA

We implemented two early phase trials in patients with recurrent malignant gliomas to evaluate vaccine safety as the primary objective, and secondarily, to assess induction of immune response against glioma-associated antigen (GAA) peptides and clinical response. These trials were designed based on our previous studies/observations as follows: 1) isolation of human leukocyte antigen (HLA)-A2-restricted CD8 + CTL epitopes derived from human GAAs; 2) development of alphaDC1, and 3) intramuscular (i.m.) administration of a toll-like receptor-3 ligand poly-ICLC remarkably enhance the effect of vaccines in brain tumor-bearing mice.

In a pilot trial UPCI 04-136, HLA-A2 + patients were vaccinated with alphaDC1s loaded with four synthetic GAA epitopes every 2 weeks by ultrasound-guided intranodal (i.n.) injections. All three patients successfully received 4 i.n. injections of GAA peptide-loaded alphaDC1 vaccines with no evidence of progression and no adverse events nor need for corticosteroids during the therapy. Positive tetramer-response against IL-13Ralpha2 and EphA2-derived peptides was observed in one patient. Tumor tissues obtained from one patient with radiological progression demonstrated mostly inflammatory response, but not many tumor 
cells. Real-time PCR demonstrated expression of vaccine-targeted GAAs in pre-vaccine, but not in the post-vaccine tumor tissues from this patient.

We next implemented UPCI 05-115, in which i.m. injections of poly-ICLC are added to the regimen employed in UPCI-04-136. The first patient with recurrent glioblastoma received 4 alphaDC1 vaccination and a total 16 i.m. poly-ICLC injections with no adverse events, and exhibited partial radiological response. While preliminary, these data support safety and feasibility of polyICLC assisted alphaDC1-based vaccines.

\section{Immunomonitoring}

\section{P097 \\ Immune monitoring for anti-cancer dendritic cell (DC) vaccine : significance of CD16 + CD56low NK cells and CD4 + CD25 $+\mathrm{T}$ cell modulation}

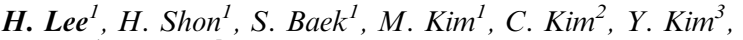
S. Kim ${ }^{4}, \mathrm{H} . \mathrm{Kim}^{5}$;

${ }^{1}$ The Cancer Center, Samsung Medical Center, Seoul, Republic of Korea, ${ }^{2}$ Department of Urology, Asan Medical Center, Seoul, Republic of Korea, ${ }^{3}$ Department of Obstetrics \& Gynecology, Asan Medical Center, Seoul, Republic of Korea, ${ }^{4}$ Department of Oncology, Asan Medical Center, Seoul, Republic of Korea, ${ }^{5}$ Stem cell Institute, FCB-Pharmicell Co. Ltd., Seoul, Republic of Korea
\end{abstract}

In the DC vaccine trial for cancer, immunological output and clinical responses are considered as major parameters to appreciate the trial results. In general the activity of tumor-specific Cytotoxic $\mathrm{T}$ cell (CTL) was detected as immune monitoring parameter however the clinical relevance was known to be weak. In this on going phase I/II clinical trial, we observed the alteration of activity or number of CD16 + CD56low cytotoxic NK cells and $\mathrm{CD} 4+\mathrm{CD} 25+$ regulatory $\mathrm{T}$ cells $(\mathrm{Tr})$ along with tumorspecific CTL to search better monitoring parameter. Six renal cell ca.(RCC), 1 breast ca. and 4 ovarian ca. pts were treated with autologous CD34 + hematopoietic stem cell-derived DC pulsed with autologous tumor cell lysate and $\mathrm{KLH}$, twice with 4 week intervals. Following each subcutaneous injection of DC vaccine, low dose (200MIU) IL-2 was introduced for 14 consecutive days as an immune adjuvant. Comparing before and 4 weeks after the final DC vaccination, $K L H$-specific in vitro $\mathrm{T}$ cell proliferation, as well as number of IFN- $\gamma$ secreting CD8 + cells were induced in 10/ 11 pts. NK activity (cytotoxicity test against Cr51 labeled-K562 cell line) was enhanced in $7 / 11$ patients $(320-2,000 \%$ of before DC vaccination control) with increase of CD16 + CD56low cytotoxic NK cell proportion. Alteration of CD $4+$ CD25 + Tr proportion was decreased in 2 RCC pts while increased in the breast and ovarian ca. pts. Significance of present data will be discussed to verify the relevance of immune parameters with clinical responses of DC vaccination.

\section{P098}

Melanoma vaccine-induced autoimmune versus tumor-specific responses: immunomonitoring of a clinical protocol in the absence of defined antigens

A. Machlenkin, S. Frankenburg, O. Elias, I. Ben David, T. Hamburger, L. Kadouri, T. Peretz, M. Lotem; Hadassah-Hebrew University Hospital, Jerusalem, Israel
Measurable parameters of immunity are especially needed when clinical response rates are low. Evaluating immune responses when the antigens involved are variable and undefined represents a particular challenge, since the most sensitive monitoring assays are based on defined antigens, usually HLA-restricted. In a previous study, we reported a strong correlation between delayed type hypersensitivity (DTH) skin reactions and prolonged survival of melanoma patients receiving an autologous whole cell vaccine. In the present study, the immune response of these patients before and after treatment was evaluated. To this end, PBMC from 33 patients, cryopreserved before and after immunization, were sensitized with autologous melanoma, followed by measurement of anti-melanoma and anti-PBMC reactivity. Interferon $-\gamma$ secretion by $\mathrm{CD} 4+$ and $\mathrm{CD} 8+\mathrm{T}$ cells (ELISPOT and ELISA), and cytolytic capacity of CD8 $+\mathrm{T}$ cells (CD107a mobilization assay) were evaluated. Autologous melanoma vaccination increased autoimmune anti-PBMC responses in the majority of patients. The strength of vaccineinduced autoimmunity was more marked in DTH + than in DTH- patients, and was associated mainly with $\mathrm{CD} 4+$ responses. Melanoma-specific $\mathrm{CD} 8+$ and $\mathrm{CD} 4+$ responses were detected in some patients, but did not correlate with DTH. This study demonstrates the feasibility of immunomonitoring in the absence of specific antigens. The results suggest that autoimmune, but not anti-melanoma responses, correlate with DTH, and thus may predict improved survival of vaccinated melanoma patients.

\section{P099}

\section{Increased immunoglobulin levels in patients responding to DC immunotherapy.}

\author{
N. L. Wilson, A. M. Gravett, J. Copier, A. G. Dalgleish, \\ M. D. Bodman-Smith; \\ St Georges University of London, London, UK
}

This study investigates the levels of immunoglobulin in serum from patients receiving autologous dendritic cell (DC) therapy for advanced metastatic melanoma and proposes that $\mathrm{DC}$ vaccination may influence the generation of subtypes of $\mathrm{IgG}$.

Dendritic cells were generated from monocytes isolated from patients and differentiated using GM-CSF and IL-4, then pulsed with tumour lysate for $2 \mathrm{~h}$. The lysate used was derived from allogeneic tumour cell lines expressing at least four shared antigens (MAGE, MART-1, gp100 and tyrosinase) After pulsing the loaded DCs were re-administered to the patient. Half of the DC vaccine (approx 1-2 $10^{6} \mathrm{DC}$ ) was given intra-tumourally or nodally, the second half intra-dermally. The vaccine was administered fortnightly for five vaccines and monthly for the remaining four.

Serial serum samples were taken from 25 patients starting before the first vaccination and then at various time points post vaccine. The levels of immunoglobulin M; A; E; G1; G2; G3 and G4 present in the serum was assessed using multi-analyte bead technology and analysed by Luminex.

We show a significant increase in the levels of $\operatorname{IgG} 1$ and $\mathrm{IgG} 2$ $(P=0.007$ and 0.028 respectively, student's $t$-test Bonferony corrected) in those patients deemed to have responded clinically to the DC therapy (stable disease) when compared to non-responders (progressive disease). Other immunoglobulin types showed no change.

These data suggest an increased, subtype specific, antibody response in patients responding to DC therapy. 


\section{P100}

HPV 16-associated tumours: in vitro effects of combined chemo-immunotherapy

\author{
M. Indrová ${ }^{1}, J$. Bieblová ${ }^{1}$, E. Pajtasz-Piasecka ${ }^{2}, J$. Śmová $^{1}$, \\ M. Reinišs ; \\ ${ }^{1}$ Institute of Molecular Genetics AS CR, v.v.i., Prague 4, Czech \\ Republic, ${ }^{2}$ Institute of Immunology and Experimental Therapy, \\ Polish Acad. Sci., Wroclaw, Poland
}

Moderately immunogenic HPV 16-associated tumours mimicking human HPV 16-associated neoplasms (MHC class I-positive tumour TC- 1 , its variant $\mathrm{TC}-1 / \mathrm{P} 3 \mathrm{C} 10$ with decreased $\mathrm{MHC}$ class I expression and MHC class I-deficient TC-1/A9, and MHC class Ideficient tumour MK16/1/IIIABC), were used to examine the effect of local interleukin 12 (IL-12) gene therapy with genetically modified tumour cell vaccines for the treatment of minimal residual tumour disease (CMRTD), obtained after cytoreductive chemotherapy with ifosfamide derivative CBM-4A. Intraperitoneal CBM-4A treatment of tumour-bearing mice exhibited a significant tumour-inhibitory effect and resulted in strong upregulation of immature myeloid $\left(\mathrm{Gr}-1^{+} / \mathrm{CD} 1 \mathrm{~b}^{+}\right)$cells in the spleens of treated mice. During the growth and treatment of tumours, downregulation of the $\mathrm{CD}^{+}$and $\mathrm{CD} 8^{+}$subpopulations in the spleens was observed, but the amount of $\mathrm{CD} 25^{+} \mathrm{CD} 4{ }^{+}$Treg cells remained unchanged. Further, the combined chemo-immunotherapy compared to chemotherapy only led to the upregulation of the IL-2, IFNgamma, TNFalpha and IL-5. In the spleens of animals treated with TC-1 (MHC class I ${ }^{+}$) but not with of TC-1/A9 (MHC class I') tumours, we found cytotoxic $\mathrm{CD} 8^{+} \mathrm{T}$ lymphocytes. These findings indicate the relevance for the treatment of CMRTD with genetically modified cellular vaccines.

Grants: 301/06/0774 (GA CR), Joint project PAN and AS CR (2006-8)

\section{P101}

\section{Generation of functional HLA-DR*1101 tetramers to characterize CD4 + T cells specific for MAGE-A3 Tumour associated antigen}

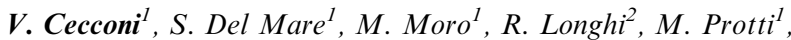 \\ P. Dellabona ${ }^{1}$, G. Casorati ${ }^{1}$; \\ ${ }^{1}$ DIBIT H San Raffaele, Milan, Italy, ${ }^{2}$ Istituto di Chimica \\ del Riconoscimento Molecolare, Milan, Italy
}

We aim at characterizing the spontaneous CD4 $+\mathrm{T}$ cell responses specific for the MAGE-3 tumour antigen in cancer patients. To the purpose, we generated fluorescent HLA-DR*1101 tetramers that can be loaded with the desired peptides to stain specific $\mathrm{T}$ cells. By combining bioinformatics and cellular immunology, we identified several MAGE-3-derived promiscuous CD4 epitopes. Four epitopes were selected for further characterisation: all peptides could activate specific $\mathrm{T}$ cells when presented by plasticbound HLADR1101 monomers, however, only MAGE-3191-205 (p39) epitope could stain specific T cells when loaded onto HLADR*1101 tetramers. These findings suggested that all peptide bound HLA-DR*1101 monomers, but their specific mode of binding was determining the tetramer capacity to stain $\mathrm{T}$ cells. We hypothesized that peptides must bear a single anchor residue to bind the HLA-DR allele in a homogeneous frame, to increase the overall avidity of the tetramer by generating four arms with identical peptide-HLA-DR complexes. Bioinformatic analysis confirmed that MAGE-3 p39 contained a single putative anchor residue, whereas the other three epitopes contained multiple ones. By identifying the minimal epitopes corresponding to these three MAGE-3 peptides, and generating minimal peptide analogues containing a single anchor residue, we indeed produced functional HLA-DR*1101 tetramers. These findings indicate a strategy to predict the structure of promiscuous peptides to generate functional HLA-DR tetramers.

\section{Immune Escape Mechanisms}

P102

Tumor associated macrophages display a unique population with features of macrophages and dendritic cells inducing regulatory $\mathbf{T}$ cells

J. C. Lehmann, K. Gurney, D. M. Danilenko, W. Lee, P. Godowski; Genentech Inc., South San Francisco, CA, USA

High levels of macrophages within breast tumors correlate with increased angiogenesis and poor prognosis. To understand the pathogenic role of tumor associated macrophages (TAM), we analyzed recruitment and function of myeloid cells in syngeneic and genetic murine cancer models. Although we observed myeloid infiltrates in all models, mature TAM were found only in the genetic models (MMTV.PyMTtg and MMTV.Her2tg). Gene expression profiling suggest that TAM are (1) distinct from other tissue macrophages in that they express markers of both macrophage and dendritic cells (DC), (2) important for inducing tolerance to the tumor and (3) up-regulate genes important for angiogenesis. In vitro, TAM are as potent as DC to stimulate $\mathrm{T}$ cell proliferation. However, unlike conventional immature DC, TAM potently induce two subsets of inhibitory T cells: IL-10 expressing Tr1 cells and FoxP $3+$ regulatory T cells. In addition, TAM induce THIL17 cells, which may contribute to angiogenesis. The relevance of these findings is strengthened by increased ratios of these three $\mathrm{T}$ cell subsets in tumor mice. Furthermore, TAM express an unique chemokine/cytokine pattern, that might contribute to the tolerogenic microenvironment of the tumor. Thus, TAMs are a novel myeloid subset functionally distinct from other macrophages or DC, which contribute to tumor tolerance. Understanding the function of TAM and their mode of action to promote tumor growth and metastasis may provide novel and innovative therapeutic strategies in the constant demand to help tumor patients.

\section{P103}

Correcting defective extracellular calcium responsiveness in chronic lymphocytic leukemia B cells - correlation with enhanced immunogenicity

C. M. Hammond ${ }^{1,2}$, D. White ${ }^{1}$, J. Tomic ${ }^{1,2}$, Y. Shi ${ }^{1}$, D. Spaner ${ }^{1,2}$; ${ }^{1}$ Sunnybrook Research Institute, Toronto, ON, Canada,

${ }^{2}$ University of Toronto, Toronto, ON, Canada

The failure of most cancers to be cured by conventional chemotherapy, along with tumour regression associated with enhanced immune activity, has motivated the development of immunotherapies, such as cancer vaccines. Therapeutic cancer vaccines have the 
ability to increase the number and activity of tumour-reactive $\mathrm{T}$ cells. However, cancer cells, such as chronic lymphocytic leukemia (CLL) B cells, express low levels of CD80, CD86 and CD83, and produce immunosuppressive factors (such as IL-10), which may prevent sustained effector function of tumour-reactive $\mathrm{T}$ cells. Accordingly, CLL immunotherapy might achieve more success if CLL cells rendered more immunogenic (i.e. increased CD83 expression and anti-inflammatory cytokine production).

We recently found that healthy $\mathrm{B}$ cells respond to calcium changes in their environment by a receptor-mediated mechanism that causes the release of calcium from intracellular stores and the activation of signaling cascades, leading to the NF-kappaB-mediated expression of CD83 and enabling them to prepare to engage in immune responses. CLL cells, however, are unable to release calcium from intracellular stores in response to extracellular calcium (resulting in negligible CD83 expression), which may be involved in their immune escape and disease progression. By inhibiting the chronic JNK activity in CLL cells, signaling between the calcium sensing receptor and intracellular calcium stores is restored and CLL cell immunogenicity is enhanced. While directed mainly to CLL, the results suggest that strategies to inhibit JNK in malignant $B$ cells may tip the balance of the immune system, improving the efficacy of $\mathrm{T}$ cell based immunotherapies.

\section{P104 \\ Prolongation of Interferon (IFN)-mediated STAT3 activation by phobol esters renders Chronic Lymphocytic Leukemia (CLL) B cells less immunogenic}

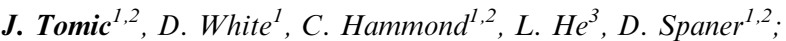 \\ ${ }^{1}$ Sunnybrook Research Institute, Toronto, ON, Canada, \\ ${ }^{2}$ Department of Medical Biophysics, University of Toronto, \\ Toronto, ON, Canada, ${ }^{3}$ Immunology platform, Sanofi-Pasteur, \\ Toronto, ON, Canada
}

CLL is the most frequent form of adult leukemia in the Western World and is incurable with conventional chemotherapy. Several lines of evidence suggest that $\mathrm{T}$ cell-mediated immunotherapy may be an effective modality for CLL. However, the intrinsically weak immunogenicity of CLL cells may inhibit this treatment. Consequently, cytokines that enhance the immunogenicity of CLL cells might be useful in improving immunotherapeutic approaches to this disease. Our laboratory has provided evidence that interferon (IFN)- $\alpha 2 b$ enhances the effectiveness of immunotherapies for malignant melanoma. In order to support the potential use of IFN in CLL, we first examined the effects of this cytokine on a number of immunogenic properties of CLL cells (i.e. costimulatory molecule expression, cytokine production, and ability to stimulate $\mathrm{T}$ cell proliferation). The results indicated that IFN had only mild effects on CLL cell immunogenicity in vitro and suggested that additional measures were needed for its use in CLL.

IFN activates primarily the transcription factor STAT1 (a tumor suppressor). A minor pathway of IFN-signalling is through STAT3 (an oncogene). We have found that the concomitant treatment with phorbol esters (PKC modulators) enhances the minor pathway. The mechanism appeared to involve inhibition of tyrosine phosphatases, which limit the extent of IFN-mediated STAT3 activation. Possibly as a result of tipping the balance towards STAT3, CLL cells remained weakly immunogenic. Specific abrogation of STAT3 phosphorylation resulted in stronger immunogenicity of CLL cells in vitro. Therefore, PKC agonists and IFN-mediated STAT3 phosphorylation inhibitors may be a clinical strategy to improve CLL immunogenicity.

\section{P105}

Blockade of PD-1 surface expression using siRNA improves immune functions of tumor-specific $\mathbf{T}$ cells

\author{
L. Borkner ${ }^{1}$, K. Schuster ${ }^{1}$, R. Andreesen ${ }^{1}$, A. Mackensen $^{1}$, \\ C. U. Blank ${ }^{1,2}$; \\ ${ }^{1}$ The University of Regensburg, Dept. Hematology/Oncology, \\ Regensburg, Germany, ${ }^{2}$ The Netherlands Cancer Institute - Antoni \\ van Leeuwenhoek Ziekenhuis (NKI-AVL), Amsterdam, \\ The Netherlands
}

Despite of sufficient homing to tumor sites, tumor associated antigen (TAA)-specific T cells seldom control tumor growth. We and others have shown that Programmed-death receptor-ligand 1 (PD-L1, B7-H1) on tumor cells plays an important role in this kind of tumor escape via interaction with Programmed death receptor-1 (PD-1) on the T cells. PD-1 has been shown to inhibit lymphocyte cytokine production and proliferation, and PD-1 gene-deficient $\mathrm{T}$ cells showed superior tumor growth control in animal models. In addition, we observed enhanced cytokine production and lytic function from human TAA-specific T cells targeting PD-L1-expressing tumors, when blocking PD-L1/PD-1 interaction. Therefore, the disruption of the PD-L1/PD1 pathway might be a promising approach to the creation of superior TAAspecific T cells. In regard of a possible clinical approach our goal is to suppress the expression of the PD-1 receptor exclusively on the TAA-specific $T$ cells by means of RNA interference. Transient transfection of EL4 lymphoma cells and antigen-activated T cells with double stranded short interfering RNA (siRNA) induced a considerable knockdown of PD-1. Retroviral transduction reached almost the levels of $\mathrm{PD}$-1-gene-deficient $\mathrm{T}$ cells, did not alter other surface molecule expressions, and resulted in improved $\mathrm{T}$ cell cytokine production. Furthermore, human B cell line C1RA2 and MelanA-specific T cells could be forced to decreased surface expressions of PD-1 or CTLA- 4 by the adequate siRNAs. In summary, using siRNA to inhibit the expression of negativecostimulatory molecules on tumor-specific $\mathrm{T}$ cells might improve the efficiency of anti-tumor immune responses either induced by vaccination or by ACT.

\section{P106 \\ Infection of mature DCs with $\mathrm{HSV}-1$ interferes with the IFN signaling pathway}

\author{
J. Eisemann, P. Muhl-Zurbes, A. Steinkasserer, M. Kummer; \\ Department of Dermatology, Erlangen, Germany
}

It has been shown that herpes simplex virus type 1 (HSV-1) blocks specific immune responses by various mechanisms. Cell lines infected with HSV-1 for instance show a severe impairment of the interferon-gamma (IFN- $\gamma$ ) induced STAT1 signaling pathway. Thus, we examined the influence of HSV-1 infection on the IFN- $\gamma$ signal transduction in mature dendritic cells (mDCs).

In this study, we report the downregulation of the IFN- $\gamma$ receptor $\alpha$ chain (IFNGR1) on mRNA level in HSV-1 infected mDCs. As a consequence, the expression of the IFNGR1 subunit on the cell surface of the infected cell was strongly reduced. Furthermore, we were able to show the inhibition of STAT1 phosphorylation following HSV-1 infection in mDCs, while protein levels of STAT1 remained constant. As a direct downstream effect of STAT1 phosphorylation, the activation of the transcription factor IRF-1 was also clearly inhibited and could no longer be induced by the addition of IFN- $\gamma$. Additional experiments using a virus strain lacking the $v h s$ gene showed that the 
mutant virus is more sensitive to IFN- $\gamma$ as STAT1 signaling was inhibited to a lesser extent. Infection with a UV-inactivated virus did not influence the STAT1 signaling pathway at all. In conclusion, we show that HSV-1 blocks IFN- $\gamma$ signaling in mDCs. We further provide evidence for the involvement of the viral protein vhs in the mechanism. Finally, a replication incompetent, UV-inactivated virus did not inhibit STAT1 phosphorylation, indicating that viral gene expression is neccessary to exert this effect.

\section{P107}

\section{Herpes Simplex Virus Type 1 (HSV-1) Induces CD83 Degradation in Mature Dendritic Cells with Immediate Early Kinetics via the Cellular Proteasome}

\author{
M. Kummer ${ }^{l}$, N. M. Turza ${ }^{1}$, P. Muhl-Zurbes ${ }^{1}$, M. Lechmann ${ }^{2}$, \\ C. Boutell ${ }^{3}$, R. S. Coffin ${ }^{4}$, R. D. Everett ${ }^{3}$, A. Steinkasserer $^{1}$, \\ A. Prechtel ${ }^{1}$; \\ ${ }^{1}$ Department of Dermatology, Erlangen, Germany, \\ ${ }^{2}$ Princess Margaret Hospital, Toronta, ON, Canada, \\ ${ }^{3}$ MRC Virology Unit, Institute of Virology, Glasgow, UK, \\ ${ }^{4}$ BioVex Ltd., Oxford, UK
}

Mature Dendritic Cells (DCs) are the most potent antigen-presenting cells within the human immune system. However, Herpes simplex virus type 1 (HSV-1) is able to interfere with DC biology and to establish latency in infected individuals. In this study we provide new insights into the mechanism by which HSV-1 disarms DCs by the manipulation of CD83, a functionally important molecule for DC activation. FACS analyses revealed a rapid downmodulation of CD83 surface expression within 6-8 h after HSV-1 infection, in a manner strictly dependent on viral gene expression. Soluble CD83 ELISA assays together with Western blot analysis demonstrated that CD83 rapidly disappears from the cell surface after contact with HSV-1 by a mechanism that involves protein degradation rather than shedding of CD83 from the cell surface into the medium. Infection experiments with an ICP0 deletion mutant demonstrated an important role for this viral Immediate-Early protein during CD83 degradation, since this particular mutant strain leads to strongly reduced CD83 degradation. This hypothesis was further strengthened by cotransfection of plasmids expressing CD83 and ICP0 into 293T cells, which led to significantly reduced accumulation of CD83. In strong contrast, transfection of plasmids expressing CD83 and a mutant ICP0 defective in its RING finger-mediated E3 ubiquitin ligase function did not reduce CD83 expression. Inhibition of the proteasome, the cellular protein degradation machinery, almost completely restored CD83 surface expression during HSV-1 infection, indicating that proteasome-mediated degradation and HSV-1 ICP0 play crucial roles in this novel viral immune escape mechanism.

\section{P108 \\ Tumour antigen specific Treg enriched in peripheral blood of breast cancer patients control natural tumour specific type-1 CD4 and CD8 $\mathrm{T}$ cell responses}

\author{
Y. G $\boldsymbol{e}^{1}$, H. Frebel ${ }^{1}$, F. Schütz ${ }^{2}$, P. Beckhove ${ }^{1}$; \\ ${ }^{I}$ The German Cancer Research Center, Heidelberg, Germany, \\ ${ }^{2}$ Department of Gynecology and Obstetrics, University hospital \\ of Heidelberg, Germany
}

Spontaneous immune responses against tumor antigens occur in many breast cancer patients and lead to an enrichment of $\mathrm{CD} 4^{+}$ and $\mathrm{CD} 8{ }^{+}$tumour-reactive memory $\mathrm{T}$ cells (memTCs) in the bone marrow (BM). Their appropriate reactivation ex-vivo by tumourantigen pulsed autologous dendritic cells and subsequent adoptive transfer resulted in complete rejections of autologous breast carcinomas in xenotransplant mouse models in vivo and respective strategy is currently under clinical evaluation. The insufficient reactivation of the natural repertoire of tumour-reactive memTCs in cancer patients remains unclear. Here, we investigated a potential impact of regulatory $\mathrm{T}$ cells (Treg) on the function of tumour-reactive memTCs in 22 breast cancer patients. Compared to $\mathrm{BM}$, the peripheral blood (PB) of tested patients contained higher amounts of $\mathrm{CD} 4{ }^{+} \mathrm{CD} 25^{+} \mathrm{FOXP} 3{ }^{+}$Treg as determined by flow cytometry $(3.4+1.1 \%$ vs. $1.9+3.1 \%, P<0.0001)$. These Treg appeared to be tumour specific, as stimulation by breast tumour antigen but not irrelevant antigens strongly inhibited the proliferation of polyclonally stimulated autologous $\mathrm{T}$ cells. They also exerted a strong inhibition of IFN- $\gamma$ secretion by tumourreactive type-1 CD4 and $\mathrm{CD} 8 \mathrm{~PB}$ memTCs, since the proportion of patients containing tumour-reactive memTCs increased significantly from 19 to $75 \%$ after Treg depletion as determined by short-term IFN- $\gamma$ Elispot. Tumour specific IFN- $\gamma$ secretion was also inhibited by addition of autologous, tumour antigen-activated Treg in a ratio of 1:8 Treg: nonTreg. We conclude that the PB of breast cancer patients is enriched for tumour-specific Treg that control the re-activation of natural tumour-specific CD4 and CD8 memTCs present in most breast cancer patients.

\section{Dendritic Cells}

\section{P109}

Plasticity of dendritic cell function in response to prostaglandin $\mathrm{E}_{2}\left(\mathrm{PGE}_{2}\right)$ and interferon-gamma (IFN- $\gamma)$.

\author{
M. Lehner, A. Stilper, P. Morhart, W. Holter; \\ Childreńs University Hospital, Erlangen, Germany
}

Current evidence suggests that maturing DCs depending on $\mathrm{PGE}_{2}$ acquire either a migratory phenotype to induce T-cell responses in lymph nodes or a pro-inflammatory phenotype to condition the microenvironment at peripheral sites. We describe a detailed analysis of the impact of $\mathrm{PGE}_{2}$ and IFN- $\gamma$ on the migratory and secretory behavior of Toll-like receptor matured human DCs and show dramatic changes in these features in a previously unrecognized manner. We propose four prototypic functional phenotypes and find, that TLR activation by R-848 in presence of IFN- $\gamma$ and $\mathrm{PGE}_{2}$ generates DCs integrating migratory and secretory function. Such dual functional DCs produced high levels of IL-12p70 and IL-23 and within $10 \mathrm{~h}$ started migration towards CCL19 and still continued to secrete substantial amounts of primary IL-12p70 after migration. The accelerated onset of migration was due to $\mathrm{PGE}_{2}$ and was associated with reduced plastic adherence and lower amounts of activated $\beta_{1}$-integrin (CD29). In contrast, IFN- $\gamma$ enhanced cell adhesion and despite induction of CD38 hindered CCR7 mediated migration.

These data suggest that appropriately co-stimulated DCs can rapidly migrate towards lymph nodes and carry with them a wave of primary cytokines. This could explain how "migratory type" DCs could possibly take part in cytokine supported differentiation of nodal $\mathrm{T}$ cells and, moreover, it is highly relevant for the development of DC vaccines. Finally, our data suggest a whole new role for 
IFN- $\gamma$ in the direct regulation of DC migration through enhanced cell adhesion perhaps to support the development of $\mathrm{T}$ cell effector functions at peripheral sites.

\section{P110 \\ Antigen release kinetics in the phagosome is critical to cross-presentation efficiency}

\author{
S. W. Howland, J. Mata-Fink, K. D. Wittrup; \\ Massachusetts Institute of Technology, Cambridge, MA, USA
}

Cross-presentation of exogenous antigens in MHC class I molecules by dendritic cells is the underlying basis for many developing immunotherapies and vaccines. In the phagosome-to-cytosol pathway, antigens in phagocytosed particles must become freely soluble before being exported to the cytosol, but the kinetics of this process has yet to be fully appreciated. We demonstrate with a yeast vaccine model that the rate of antigen release in the phagosome directly affects cross-presentation efficiency, with an apparent time limit of about 25 min post-phagocytosis for antigen release to be productive. Three distinct methods of manipulating antigen release kinetics and their effects on cross-presentation are presented here.

\section{P111 \\ Regulation of dendritic cell function by histone deacetylase inhibitors}

\author{
J. Beck ${ }^{1}$, A. Nencioni ${ }^{2,3}$, F. Grünebach ${ }^{1}$, A. Bringmann ${ }^{1}$, \\ L. Kanz ${ }^{1}$, F. Patrone ${ }^{2}$, A. Ballestrero ${ }^{2}$, P. Brossart ${ }^{1}$; \\ ${ }^{1}$ Abteilung für Hämatologie, Onkologie, Immunologie, \\ Rheumatologie und Pulmologie, Universitätsklinikum Tübingen, \\ Tübingen, Germany, ${ }^{2}$ Department of Internal Medicine, University \\ of Genova, Genova, Italy, ${ }^{3}$ Whitehead Institute for Biomedical \\ Research, Cambridge, MA, USA
}

Histone deacetylase (HDAC) inhibitors have promising antitumor activity and are presently explored in clinical studies for malignancies of different histology. Here, we show that two HDAC inhibitors of clinical use, sodium valproate and MS-275, profoundly affect cytokine-induced differentiation of human monocytes to dendritic cells (DCs), and impair DC phenotypic maturation in response to the Toll-like receptor (TLR) ligand polyinosinic:polycytidylic acid (poly I:C). HDAC inhibitors reduce cytokine secretion and immunostimulatory capacity of DCs and inhibit DC migration toward the chemoattractant CCL19/MIP-3 $\beta$. The observed defects in DC function were not due to an increased rate of apoptosis or production of IL-10. We finally investigated the effects of HDAC inhibition on signalling pathways that play a key role in DC differentiation and function. HDAC inhibitors have been reported to cause downregulation of Bcl-6 and the hematopoietic transcription factor PU.1. However, no significant reduction in protein levels of these factors could be detected in DCs treated with VPA. In contrast, addition of VPA blocked RelB nuclear relocalization in response to poly $\mathrm{I}: \mathrm{C}$ in a concentrationdependent manner. Interestingly, the nuclear levels of IRF-3 and IRF-8, which are involved in DC differentiation, IL-12 production and DC migration were also reduced by VPA in unstimulated as well as poly I:C-activated DCs. On the other hand, IRAK-1 downregulation in response to poly I:C was not affected by VPA, suggesting that the effects of HDAC inhibition on RelB, IRF-3 and IRF-8 are mediated downstream of MyD88 and IRAK-1. In conclusion, HDAC inhibitors exhibit strong immunomodulatory properties on human DCs.

\section{P112}

\section{hDectin-1 is involved in uptake and cross-presentation of cellular antigens}

\author{
F. Grünebach ${ }^{l}$, M. M. Weck ${ }^{1}, S$. Appel $^{l}$, D. Werth ${ }^{1}$, \\ C. Sinzger ${ }^{2}$, A. Bringmann ${ }^{1}$, P. Brossart ${ }^{1}$; \\ ${ }^{1}$ Abteilung für Hämatologie, Onkologie, Immunologie, \\ Rheumatologie und Pulmologie, Universitätsklinikum Tübingen, \\ Tübingen, Germany, ${ }^{2}$ Institut für Medizinische Virologie, \\ Universitätsklinikum Tübingen, Tübingen, Germany
}

Human (h)Dectin-1 is a member of the C-type-lectin-like receptor family that was shown to be the major receptor for fungal $\beta$ glucans and to play an important role in the cellular responses mediated by these carbohydrates. It is mainly expressed on human DCs and macrophages.

In our study, we observed that activation of monocyte-derived dendritic cells (MDCs) with TLR3 ligand (poly I:C) resulted in down-regulation of hDectin-1 expression and this down-regulation correlated with a reduced uptake of apoptotic cells in phagocytosis assays. The involvement of hDectin-1 in phagocytosis was further confirmed by the addition of the recombinantly expressed extracellular domain of hDectin-1 or hDectin-1-specific linear $(1 \rightarrow 3)$ - $\beta$-D-glucan polymer curdlan to the assays that led to a significant reduction of the uptake of apoptotic cells.

Furthermore, IFN- $\gamma$ ELIspot assay, using a cytomegalovirus (CMV)-pp65 specific CTL line, revealed that hDectin-1 is also involved in the uptake of CMV-infected human fibroblasts leading to cross-presentation of CMV-derived peptides on MHC class I molecules and activation of CMV pp65-specific CD8 + T-lymphocytes. These findings were verified by the addition of the inhibitors of the endogenous pathway of antigen presentation lactacystin or brefeldin $\mathrm{A}$, as these agents almost completely abolished the lysis of MDCs incubated with CMV + fibroblasts in 51Cr-release assays.

Summarized, the data reported here show that hDectin-1 functions not only as a pattern recognition receptor in innate immunity but is also involved in the clearing of apoptotic cells and cross-presentation of cellular antigens on MHC class I molecules to specific CTLs.

\section{P113}

\section{Characterization of dendritic cell-specific genes p275 and p306}

\author{
A. Bringmann ${ }^{l}$, I. Hilgendorf ${ }^{l}$, D. Kurz ${ }^{1}$, S. Appel $^{2}$, \\ F. Grünebach ${ }^{1}$, L. Kanz ${ }^{1}$, P. Brossart ${ }^{1}$; \\ ${ }^{1}$ Abteilung für Hämatologie, Onkologie, Immunologie, \\ Rheumatologie und Pulmologie, Tübingen, Germany, \\ ${ }^{2}$ Institute for Biomedicine, Bergen, Norway
}

Using a substractive cDNA library based on suppression hybridization between monocyte derived DC (mDC) cDNA and the reference monocyte cDNA, we identified two differentially in mDC expressed genes p275 and p306. p275 codes for a membrane protein and probably represents a splice isoform of the transport protein NAT-1. The predicted structure of protein p306 is globular, suggesting that the protein is either intracellular or secreted. The expression of both genes was confirmed by RT-PCR using cDNA isolated from peripheral blood monocytes and $\mathrm{DC}$, generated in vitro from monocytes or $\mathrm{CD} 34+$ progenitor cells. 
To further analyze the protein expression polyclonal antibodies were generated by immunization with synthetic peptides deduced from the identified sequences. Interestingly, inhibition of DC differentiation using IL-10 or STI571 (Imatinib) resulted in an impaired expression of both proteins.

Utilizing specific primers for two recently described splice variants of p306 we identified a new splice form expressed in $\mathrm{mDC}$.

Analyzing different DC populations in peripheral blood we show that p306 is expressed in LPS-activated DC isolated from peripheral blood.

Although the functions of p275 and p306 in mDC have yet to be determined, both genes play a role in $\mathrm{mDC}$ differentiation. Especially p306 might be an interesting marker as the predicted protein does not resemble any known protein structure.

\section{P114 \\ Antigen delivery to dendritic cells by receptor mediated endocytosis}

\section{Staudinger, M. Peipp, M. Gramatzki, R. Repp; Division of Stem Cell Transplantation and Immunotherapy, Kiel, Germany}

Dendritic cells (DC) are attractive candidates for anti-tumor vaccination strategies, as they are able to induce and regulate adaptive immune responses. In contrast to antigen uptake from the fluid phase, receptor mediated endocytosis of immunocomplexes would lead to an activation of both, T cell help and cytotoxic $\mathrm{T}$ cells, by cross presentation. To establish a protocol for an efficient antigen delivery to DCs, we comparatively investigated cells exhibiting a DC-like phenotype derived in vitro from isolated monocytes of healthy donors by cultivation using three different sets of cytokines (IL4/GM-CSF, IFN $\alpha / G M-C S F$, or IFN $\beta /$ IL3). These DCs have been analysed for their surface expression levels of $\mathrm{Fc}$ and lectin receptors useable for antigen uptake, their endocytotic capacities and their sensitivity to maturating proinflammatory cytokines or toll-like receptor ligands. To direct antigens to specific uptake receptors expressed on DCs, proteins of variable regions of antibodies specific for Fc-receptors Fc $\alpha$ RI (CD89) and Fc $\gamma$ RI (CD64) and the DCrestricted lectin receptor DC-Sign (CD209) fused to GFP or antigens have been designed, expressed in eucaryotic cell lines and purified by IMAC. Data verifying specific binding of these molecules and antigen loading of DCs will be presented. Finally, this approach may open up new perspectives to overcome in vitro manipulation of monocyte derived DCs for vaccination purposes by in vivo application of antigens specifically directed to DCs.

\section{P115}

\section{Aptamers as artificial antibodies for dendritic cells}

\section{V. Berezovski, M. Lechmann, T. W. Mak; \\ The Campbell Family Institute for Breast Cancer Research, Toronto, ON, Canada}

One of the most challenging tasks of biotechnology is the replacement of animal- or hybridoma-made antibodies with artificial competitors that can be easily selected and chemically synthesized. Aptamers are DNA or RNA single-stranded oligonucleotides that can bind to proteins on living cells with high affinity and specificity. Aptamers are affinity ligands with the potential to change the field of affinity probes and replace antibodies as diagnostic, analytical and therapeutic reagents. Aptamers have indisputable advantages over antibodies; they can be engineered completely in a test tube, are readily produced by chemical synthesis, possess desirable storage properties, and elicit little or no immunogenicity in therapeutic applications. In our work, we applied a cell-based SELEX technology to select aptamers for dendritic cells. The first pool of aptamers specifically recognized mature dendritic cells (mDC) versus immature dendritic cells (imDC). The second pool was bound well to the both type of cells and the third pool had a better affinity to imDC than to $\mathrm{mDC}$. The affinity was in the nanomolar range on ice, room temperature and $37^{\circ} \mathrm{C}$ in presence of background DNA. We also assessed the binding of the first pool by confocal microscopy. Remarkably, aptamers were bound to discrete regions on the surface of the mature dendritic cells. At the next stage, these aptamers will be used to find new biomarkers for dendritic cell activation. We also plan to identify highly specific activating or inhibitory aptamers which can be very interesting candidates to induce tolerance in transplantations.

\section{P116}

\section{The in vitro inhibitor effect of osteosarcoma cell lines on different dendritic cells subsets}

M. Muraro, O. M. Mereuta, F. Carraro, E. Madon, F. Fagioli;
Stem Cell Transplantation and Cellular Therapy Unit, Turin, Italy

Background: Dendritic cells (DCs) might be partly responsible for the defective immune response in tumor bearing hosts, but no study in osteosarcoma patients is still available. Therefore, we investigated the in vitro inhibitor effect of osteosarcoma cell lines on CD14 + DCs, DCs type 1 (DC1) and DCs type 2 (DC2).

Materials and methods: DCs populations were selected from PBMCs of healthy donors by specific magnetic isolation methods and maturated with appropriate stimuli. All DCs populations were plated with different concentration of osteosarcoma cell lines or their supernatants. After 5 or 6 days of culture we analyzed the expression of maturation markers: CD40, 80, 83, 86 and HLADR. In some cases, IL-12, CD40L and Indometacin were added to the DCs co-cultures.

Results: We found a significant decrease of the co-stimulatory molecules expression in all CD14 + DCs and DC2 experimental conditions, whereas the inhibition of DC1 maturation was different depending on the osteosarcoma cell line and ratio used. The addition of IL-12 or CD40L or Indometacin to the CD14 + DCs co-cultures partially blocked the immunosuppressive effect of the osteosarcoma cell lines. Depending on the osteosarcoma cell lines used, IL-12 and Indometacin reversed their immunosuppressive effect allowing a slight DC2 maturation. No effect was revealed on DC1 maturation.

Conclusions: Osteosarcoma cell lines highly interferes with the in vitro DCs immune function as antigen presenting cells. This better understanding of tumor biology and the avoiding of the immune system, underlines the need for new treatments to reverse this immune-surveillance inhibition for a specific osteosarcoma immunotherapy. 


\section{P117}

Sphingosine Kinase inhibitor suppresses

a Th1-polarization via the inhibition of

Immunostimulatory activity in murine bone marrow-derived dendritic Cells

\author{
I. Jung, Y. Jeong, C. Lee, Y. Park; \\ Pusan national university, Busan, Republic of Korea
}

Sphingosine kinase (Sphk) has been shown to be activated by growth factor and survival factors, and one of its products, sphingosine-1-phosphate (S1P), plays an important role in the regulation of various cellular responses. However, the effect of Sphk on the maturation and immunostimulatory function of dendritic cells (DCs) still remains largely unknown. In this study, we examined whether Sphingosine Kinase Inhibitor (SKI) can influence costimulatory molecules (CD40, CD80, CD86 and MHC class II) and cytokine production (IL-12 and IL-10) in murine bone marrowderived DCs (BMDCs). SKI significantly inhibited co-stimulatory molecules in DCs. SKI suppressed IL-12 production by DCs and IFN- $\gamma$ production by $\mathrm{T}$ cells. In addition, SKI inhibited LPS-induced the translocation of $\mathrm{NF}-\kappa \mathrm{B}$, whereas it did not affect the degradation of IL-1 receptor-associated kinase-1 (IRAK-1) by LPS. These novel findings provide new insight into the immunopharmacological role of SKI in terms of its effects on DCs. These findings open a possibility for further understanding of the immunopharmacological functions of SKI, as well as therapeutic adjuvants for the treatment of DC-related acute and chronic diseases.

\section{P118}

\section{Dendritic cells in an immune privilege}

site - characterization of maturation stage in normal and chronically inflamed rat testis

\author{
M. Fijak ${ }^{1}$, V. A. Guazzone ${ }^{2}$,L. Lustig ${ }^{2}$, W. von Wulffen ${ }^{3}$, \\ H. Hackstein ${ }^{4}$, E. Schneider ${ }^{1}$, A. Meinhardt ${ }^{1}$, C. Rival ${ }^{2}$; \\ ${ }^{1}$ Department of Anatomy and Cell Biology, JLU Giessen, Germany, \\ ${ }^{2}$ Center for Research in Reproduction, University of Buenos Aires, \\ Argentina, ${ }^{3}$ Department of Pulmonary and Critical Care Medicine, \\ University of Giessen Lung Center (UGLC), Germany, ${ }^{4}$ Institute \\ for Clinical Immunology and Transfusion Medicine, JLU Giessen, \\ Germany
}

Dendritic cells (DC) are potent antigen presenting cells and presentation of self antigens by DC is likely to play an important role in the initiation of autoimmunity and its progression. Our previous characterization of testicular autoantigens in experimental autoimmune orchitis (EAO), a model of chronic testicular inflammation, prompted us to investigate the activation state of DC in normal and EAO rat testis. Immunohistochemical and stereological analysis of Ox-62 + and CD11c + cells revealed that the number of DC in EAO testes (ca. $7 \times 10^{5} /$ testis) increased significantly compared to control rats (ca. $1 \times 10^{5} /$ testis). To understand the role the role of DC in testicular inflammation, we performed a detailed analysis of different maturation markers such as costimulatory molecules (CD80, CD86), MHCII, chemokine receptors (CCR2, CCR7) and cytokines (IL-10, IL-12p70) and developed a protocol for isolation of a highly pure fraction $(97 \%)$ of DC from the testis. We observed no significant differences in the expression of CD80, CD86 and MHC class II between groups, however, significantly elevated expression of CCR7 and a concomitant decrease of IL-12p40 subunit mRNA in the 50 day EAO group were evident. The CCR2 level in EAO animals was significantly decreased compared to adjuvant controls and expression for IL-10 and IL-12p35 subunit were detectable only in 50 day EAO group. Summarized, these results suggest that testicular DC in normal testis are not mature and tolerogenic, whereas in EAO testis DC are in a mature immunogenic state prior migration to the lymph nodes for $\mathrm{T}$ cell activation.

\section{P119 \\ Tolerogenic character of dendritic cells} in Ret-transgenic melanoma mouse

\author{
F. Zhao, D. Schadendorf, V. Umansky; \\ German Cancer Research Center (DKFZ), Heidelberg, Germany
}

Dendritic cell (DC) population is heterogeneous. Immature DCs are tolerogenic because they express low cell-surface levels of MHC molecules, CD40, CD80 and CD86. However, some tolerogenic DCs can also express maturation markers but secret less IL-12 and more IL-10.

Our aim is to investigate a role of tolerogenic dendritic cells in the spontaneous development of melanoma in Ret-transgenic mice (C57Bl/6 background), in which human proto-oncogene ret is overexpressed in melanocytes and which resembles human melanoma with respect to tumor genetics, histopathology and clinical development.

We found a significant reduction in numbers of $\mathrm{MHCII}^{+}$ $\mathrm{CD}_{11 \mathrm{c}^{+}}$cells (total DCs) as well as CD40 ${ }^{+} \mathrm{MHCII}^{+} \mathrm{CD} 11 \mathrm{c}^{+}$ cells (mature DCs) in the spleen and bone marrow (BM) of tumorbearing mice as compared to control groups (wild type or Ret-tg mice without melanoma), but not in peripheral lymph nodes. A strong increase of DCs with more immature phenotype was detected in larger tumor (more than $400 \mathrm{mg}$ ) as compared to smaller tumors (less than $400 \mathrm{mg}$ ).

When we stimulated freshly isolated CD11c ${ }^{+}$splenic DCs or DCs generated from BM with CpG1668 in vitro, DCs from tumor-bearing mice secreted more IL-10 and less IL12p70 than corresponding DCs from control mice.

In conclusion, we suggest that our data may provide an additional insight into the suppression of anti-tumor T-cell immune responses observed during melanoma progression.

\section{P120}

\section{Sequential signal delivery improves dendritic cell maturation and $T$ cell activation}

\section{T. Simon, S. Tanguy-Royer, P. Royer, M. Gregoire, Inserm U601, Nantes, France}

After capturing and processing antigens, dendritic cells (DC) migrate to lymph nodes where they activate $\mathrm{T}$ cells and generate specific immune responses. These properties have been exploited in many antitumoral immunotherapies. The positive but transitory immunological responses observed in these clinical trials point at the major question which remains unanswered: how to achieve effective maturation to successfully generate both immune benefit and clinical responses?

Yet, it is well known that helper T cells are necessary to licence and condition DC, which then become empowered to initiate a cytotoxic response. However, the immune response generated by this activated DC is actually under estimated. Thus, we developed in vitro a DC activation model mimicking maturation stimuli involved in sequential exposure that would occur in vivo. We used: 
- as first signals TNFalpha plus polyIC (inflammatory and pathogen signals)

- as second signals CD40L plus IFNgamma or activated CD4 + T cells. DC maturation states and T cell responses were assessed. Our results show that the sequential maturation process, above all with activated $\mathrm{CD} 4+\mathrm{T}$ cells, increased dramatically the DC maturation according to DC phenotype and cytokine secretion. Interestingly, Thl polarization and CTL activation was widely favoured. Furthermore, this sequential maturation system allows the generation of a long term memory $\mathrm{T}$ cell pool. Moreover, we noted an inhibiting impact on Treg population, as assessed by the decrease of FoxP $3+$ cell proportion. Thus our process should be considered in the future development of dendritic cell vaccination to tip over the balance of the immune system.

\section{P121}

\section{Fuorescence microscopy imaging to monitor Dendritic Cells' tumor lysate capturing and processing: preliminary data}

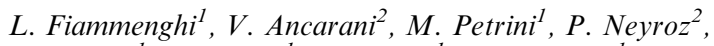
E. Pancisi ${ }^{l}$, L. Ridolf ${ }^{l}$, R. Ridolf ${ }^{l}$, A. Riccobon ${ }^{1}$;

${ }^{I}$ Immunoterapia e Terapia Cellulare Somatica Istituto Scientifico Romagnolo per lo Studio e la Cura dei Tumori, Meldola (FC), Italy, ${ }^{2}$ Dipartimento di Biochimica, Bologna, Italy

Part of the DCs cultured for therapeutic vaccination within a phase I-II clinical trial with DCs loaded with autologous tumor lysate on melanoma patients, ongoing at IRST, Meldola (FC), were used to monitor the tumor lysate capturing process by DCs. After loading with the fluorescent lysate, DCs were evaluated at four steps.

1. After $8 \mathrm{~h}$ loading (immature DC, iDC): an intensive and diffuse fluorescence was observed within the cytoplasmic compartment.

2. After $18 \mathrm{~h}$ loading (iDC): the cells' fluorescence intensity was significantly increased and lysate's fluorescence appeared to be localized in a restricted, cloudy-shaded, area of the citoplasmic compartment, with a typical polarized aspect.

3. As in step 2, plus $24 \mathrm{~h}$ maturation (mature $\mathrm{DC}, \mathrm{mDC}$ ): the cloudy area was more defined but fluorescence decreased. In addition, small fluorescent spots clearly appeared.

4. As in step 2, plus $48 \mathrm{~h}$ maturation (mDC): the fluorescence intensity of the cloudy-delimited area further decreased and the number and fluorescence intensity of the spot-like images contemporaneously increased.

Our results confirm that overnight pulsing with tumor lysate is appropriate and represents a good practice in vaccination trials with DCs.

Moreover, the morphological patterns obtained by fluorescence microscopy as a function of time from pulsing and maturation of DCs loaded with a fluorescent lysate are in excellent agreement with the recent survey published by Munz and coworkers (2007). They have shown the existence of constitutive autophagosome formation in MHC class II-positive cells. We propose that, in DCs, autologous tumor lysates may be processed and organized in autophagosomes.

\section{P122}

Improved in vitro maturation of human monocyte-derived dendritic cells with Monophosphoryl Lipid A under serum-free conditions

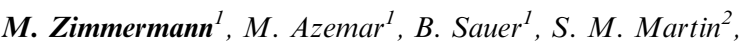

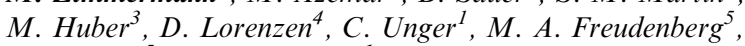 \\ C. Galanos ${ }^{5}$, B. Hildenbrand ${ }^{1}$;
}

${ }^{1}$ Tumor Biology Center, Freiburg, Germany, ${ }^{2}$ University Medical Center, Department of Dermatology, Freiburg, Germany,

${ }^{3}$ Molecular Immunology, Biology III, University Freiburg, Freiburg, Germany, ${ }^{4}$ Institut für Tumortherapy, Duderstadt, Germany, ${ }^{5}$ Max-Planck Institute for Immunobiology, Freiburg, Germany

Introduction: $\mathrm{T}_{\mathrm{H}} 1$-polarisation of matured dendritic cells $\left(\mathrm{DC}_{1}\right)$ is critical for the induction of an efficient Antigen-specific T-lymphocyte response and may be essential for the develop-ment of human vaccines relying on $\mathrm{T}$ cell immunity. Therefore, clinically approvable adjuvants for $\mathrm{DC}_{1}$ maturation are required. In this study we have tested Monophosphoryl Lipid A (MPL-A), a promising detoxificated preparation of Lipid A, to mature human monocyte-derived DC (moDC) under serum-free conditions in the presence or absence of Interferon-gamma (IFN- $\gamma$ ).

Methods: MoDC were generated in serum-free medium with GMCSF and IL-4 for 5 days and matured in different concentrations $(0.01-100 \mu \mathrm{g} / \mathrm{ml})$ for another $3,6,12,24$ or $48 \mathrm{~h}$ with MPL$\mathrm{A} \pm \mathrm{IFN}-\gamma$. Matured DC were phenotypically characterised and measured for IL-6, IL-10 and IL-12 p70 production by ELISA.

Results: We could show that MLP-A readily matures moDC in serum-free medium within 48 hours of incubation. The highest levels of IL-6 and IL-10 were achieved at a concentration of $1 \mu \mathrm{g} /$ ml. Simultaneous addition of IFN- $\gamma$ leads to significant higher levels of bioactive IL-12 and IL-6 concomitant with lower levels of IL-10. Therefore, MPL-A in combination with IFN- $\gamma$ enhances considerably the $\mathrm{T}_{\mathrm{H}} 1$-polarisation of moDC. Besides, addition of IFN- $\gamma$ resulted in more pronounced upregulation of CD83 and B7 molecules.

Conclusion: Highly purified MPL-A represents a suitable agent for the ex vivo maturation of human moDC under serum-free conditions. Simultaneous addition of IFN- $\gamma$ induces $\mathrm{T}_{\mathrm{H}} 1$-polarisation of matured moDC most efficiently. Therefore, together with IFN $-\gamma$, MPL-A seems to be a promising adjuvant for $\mathrm{DC}_{1}$-maturation.

\section{P123}

Tumour associated Tn-MUC1 glycoform is internalized through the macrophage galactose-type C-type lectin and delivered to the HLA Class I and II compartments in dendritic cells

C. Napoletano ${ }^{1}$, A. Rughetti ${ }^{1}$, M. P. Agervig Tarp ${ }^{2}$, J. Coleman ${ }^{3}$, E. P. Bennett ${ }^{4}$, G. Picco ${ }^{3}$, P. Sale ${ }^{5}$, K. Denda-Nagai ${ }^{6}$, T. Irimura ${ }^{6}$, U. Mandel ${ }^{4}$, H. Clausen ${ }^{2}$, L. Frati ${ }^{1}, J$. Taylor-Papadimitriou ${ }^{3}$, J. Burchell ${ }^{3}$, M. Nuti ${ }^{1}$;

${ }^{1}$ Department of Experimental Medicine, University of Rome "La Sapienza", Rome, Italy, ${ }^{2}$ Department of cellular and molecular medicine, University of Copenhagen, Copenhagen, Denmark, ${ }^{3}$ Breast Cancer Biology Group, Guy's Hospital, London, UK, ${ }^{4}$ School of Dentistry, Department of Oral Diagnostic, University of Copenhagen, Copenhagen, Denmark, ${ }^{5}$ Department of Cellular and Molecular Pathology, IRCCS S Raffaele, Rome, Italy,

${ }^{6}$ Laboratory of Cancer Biology and Molecular Immunology, University of Tokyo, Tokyo, Japan

The type of interaction between tumor associated antigens and specialized antigen presenting cells such as dendritic cells (DCs) is critical for the type of immunity that will be generated. MUC1, a highly $O$-glycosylated mucin, is over-expressed and aberrantly glycosylated in several tumor histotypes. This results in the expression of tumor-associated glycoforms and in MUC1 carrying the tumor specific glycan Tn (GalNAcî \pm 1 -O-Ser/Thr). Glycopeptides corresponding to three tandem repeats of MUC1, enzymatically glycosylated with 9 or 15 moles of GalNAc were shown 
to specifically bind and be internalized by immature monocyte derived DCs (iDCs). Binding required calcium and the GalNAc residue and was competed out by GalNAc polymer and Tn-MUC1 or Tn-MUC2 glycopeptides. The Macrophage Galactose-type Ctype Lectin (MGL) receptor expressed on iDCs was demonstrated to be responsible for the binding. Confocal analysis and ELISA performed on subcellular fractions of iDCs showed that the TnMUC1 glycopeptides co-localized with HLA class I and II compartments after internalization. Importantly, although Tn-MUC1 recombinant protein bound and was internalized by MGL, the glycoprotein entered to the HLA class II compartment, but not in HLA class I pathway. These data indicate that MGL expressed on iDCs is an optimal receptor for internalization of short GalNAc carrying immunogens to be delivered in HLA class I and II compartments. Such glycopeptides therefore represent a new way of targeting the HLA class I and II pathways of DCs. These results have possible implication in designing cancer vaccines.

\section{P124}

\section{Inhibitory effect of curcumin on indoleamine 2, 3-dioxygenase expression in murine dendritic cells}

\author{
Y. I. Jeong ${ }^{1}$, I. D. Jung ${ }^{2}$, C. M. Lee ${ }^{2}$, J. D. Lee ${ }^{1}$, \\ Y. M. Park ${ }^{2}$; \\ ${ }^{1}$ Department of Microbiology, Pusan National University College \\ of Natural Science, Busan, Republic of Korea, ${ }^{2}$ Department of \\ Microbiology and Immunology, and National Research Laboratory \\ of Dendritic Cell Differentiation \& Regulation, and Medical \\ Research Institute, Pusan National University College of Medicine, \\ Busan, Republic of Korea
}

Indoleamine 2, 3-dioxygenase (IDO), a key enzyme that catalyses the initial and rate-limiting step in the degradation of the tryptophan, is simultaneously expressed in murine dendritic cells stimulated with interferon- $\gamma($ IFN- $\gamma)$. More importantly, IDO has recently been established as a key enzyme in $\mathrm{T}$ cell suppression and induction of immune tolerance to tumor. In the present study, we investigated whether curcumin, which is suggested to exhibit antioxidant property, could suppress the functional expression of IDO in murine bone marrow-derived dendritic cells (BMDCs) stimulated with IFN- $\gamma$ and tumor-draining lymph nodes (TNLD) of tumor-bearing mice. Treatment with curcumin reduced the expression and activity of IDO both in IFN- $\gamma$-activated BMDCs in vitro and in $\mathrm{CD} 11 \mathrm{c}^{+} \mathrm{CD} 8 \alpha^{+}$DCs in vivo tumor-bearing mice model. Consequently, we obtained evidence that curcumin recovers the IDO-dependent $\mathrm{T}$ cell suppression. In this study, we demonstrated that curcumin inhibits activation and binding of STAT1 to the IRF-1 promoter in response to IFN- $\gamma$.

As a result of this effect, we may assume that curcumin is able to recover IDO-dependent $\mathrm{T}$ cell suppression via inhibition of IDO expression on BMDCs.

\section{P125}

In vivo evaluation of B-CLL immunotherapy with autologous and allogeneic DCs on SCID mouse model

\author{
I. Hus ${ }^{1}$, J. Kawiak ${ }^{2}$, G. Hoser ${ }^{2}$, J. Tabarkiewicz ${ }^{1}$, S. Radej ${ }^{l}$, \\ A. Dmoszynska ${ }^{1}, J$. Rolinski ${ }^{1}$; \\ ${ }^{1}$ Medical University of Lublin, Lublin, Poland, \\ ${ }^{2}$ Medical Centre of Postgraduate Education, Warsaw, Poland
}

In order to investigate in vivo therapeutic potential of DCs vaccines in B-CLL we transplanted human leukemic cells intraperitoneally into SCID mice and then try to treat the animals with human autologous or allogeneic monocyte-derived DCs pulsed with leukemic cell lysates. After 7 days from DCs injection, therapeutic effect of DC vaccines was evaluated by a measurement of CD19+/CD5 + human leukemic cells counts in the peritoneal fluid of SCID mice treated with DCs, as compared to control mice who received only PBMC of B-CLL patients. We found, that mean number of CD19+/CD5 + leukemic cells was lower in peritoneal fluid of mice treated with allogeneic $\left(140.33 \pm 152.6 \times 10^{3}\right.$ vs. $\left.197.33 \pm 203.11 \times 10^{3}\right)$ as well as autologous DCs $\left(416 \pm 656.6 \times 10^{3}\right.$ vs. $\left.580.8 \pm 738.22 \times 10^{3}\right)$ as compared to the animals not receiving immunotherapy. Response to the immunotherapy, defined as lower number of $\mathrm{CD} 19+/ \mathrm{CD} 5+$ cells in mice treated with autologous DCs $\left(283.4 \pm 532.0 \times 10^{3}\right)$ as compared to the mice, that received only B-CLL cells $\left(693.6 \pm 800.8 \times 10^{3}\right)$ was found in the animals, that were implanted with cells obtained from 5 out of 10 patients. Similar observations concerned allogeneic dendritic cells. Lower number of CD19+/CD5 + cells in peritoneal fluid of mice vaccinated with DCs $\left(75.7 \pm 66.1 \times 10^{3}\right)$ as compared to the mice receiving only B-CLL cells $\left(75.7 \pm 66.1 \times 10^{3}\right)$ was observed in the animals inoculated with cells obtained from four patients. Results obtained both with autologous, as well as allogeneic DCs were similar. It seems worth to continue the presented studies on SCID mouse model in order to improve the efficacy of DC immunotherapy in B-CLL patients.

\section{P126}

The affect of lymphoma lysate-pulsed syngeneic dendritic cells on activation of dendritic cells in tumor-bearing mice and on lymphoma process

\author{
A. L. Sevko ${ }^{1}$, M. R. Shurin ${ }^{2}$; \\ ${ }^{1}$ R.E.Kavetsky Institute of Experimental Pathology, Oncology \\ and Radiobiology NAS of Ukraine, Kyiv, Ukraine, \\ ${ }^{2}$ University of Pittsburgh Medical Center and University \\ of Pittsburgh Cancer Institute, Pittsburgh, PA, USA
}

After antigen stimulation dendritic cells (DC) move to the T-cell cites of lymph nodes and spleen. The co-stimulation events occurred between activated DC and T-cells, and the number of cytokines is produced under such interactions, like as IL 4, IL12, GM-CSF, FLT3 etc. These cytokines are favorable for DC differentiation and maturation. However, the tumor-derived factors cause the fail of DC maturation and T-cell activation. This includes down-regulation of tumor antigen processing and expression; lack of costimulatory molecules on tumor cells; release of immunosuppressive factors and others.

The aim of presented work was to study the affect of tumor antigen pulsed syngeneic DC on lymphoma process and recovering of DC maturation in tumor-bearing mice.

Methods: Immunophenotype of DC was evaluated using anti-IA ${ }^{\mathrm{b}}$, CD86, CD80, CD40, CD11c and CD11b monoclonal antibodies (Flow Cytometry); EL-4 lymphoma lysate (LL) was obtained by freezing-thawing; DC were maintained from bone marrow precursors in vitro.

Results: Partial recovering of recipients' DC was observed when LL-pulsed DC were injected intravenously (IV) and intratumorally (IT) in compare with DC from tumor-bearing mice, which were treated with naive DC or non-treated animals; the longer survival was shown in case of IV administration of LL-pulsed DC. 
Conclusion: There was observed recovering of maturation of recipients' DC with affect on EL-4 lymphoma development in IV treatment of lymphoma-bearing mice with syngeneic LL-pulsed DC. No single treatment has been successful in improving survival outcomes. This approach should be investigated for combined treatment using others, like chemotherapy, for improving results.

\section{P127}

\section{Application of tocotrienols as an adjuvant} in developing cancer vaccines

\author{
S. Abdul Hafid ${ }^{1,2}$, A. Radhakrishnan ${ }^{2}$, K. Nesaretnam ${ }^{1}$; \\ ${ }^{1}$ Malaysian Palm Oil Board (MPOB), Bandar Baru Bangi, \\ 43000 Kajang, Selangor, Malaysia, ${ }^{2}$ International Medical \\ University (IMU), Plaza Komanwel, Bukit Jalil, \\ 57000 Kuala Lumpur, Malaysia
}

Dendritic cell (DC) vaccine is a potent form of cancer immunotherapy due to the ability of DCs to process and present antigens to T-cells and stimulate specific immune responses. However, development of cancer vaccine has often been hampered by poor immunogenicity of the relevant antigenic components, which require potent adjuvants in order to efficiently promote a protective immune response. In this study, we have used Tocotrienol-rich fraction (TRF), a non-toxic natural compound as an adjuvant to induce an immune response and enhance the effectiveness of DCbased cancer vaccine to prevent tumor growth and spread in a mouse model of breast cancer. Our findings show that TRF in combination with DC pulsed with tumor lysate injected subcutaneously, significantly inhibited the growth of 4T1 mammary tumour as compared to control group and mice injected with DC alone. Frequent vaccinations in experimental mice showed further suppression of tumor growth. Analysis of mice splenocytes and mixed lymphocyte reaction (MLR) assay revealed significantly higher levels of IFN- $\gamma$ in the T-cells of mice treated with TRF and DC compared to control and mice injected with DC alone. Furthermore, CTL assay showed that subcutaneous injection of DC pulsed with tumor lysate in the presence of TRF induced tumor specific cytotoxic T-lymphocytes. This study demonstrates the potential of TRF as an adjuvant in enhancing the effectiveness of DC based vaccines in treating breast cancer in a mouse model.

\section{P128}

\section{Regulation of phagocytosis in dendritic cells:} A gene transfection approach

\author{
S. Wong ${ }^{1}, Y . \mathrm{Ho}^{1}, \mathrm{C} . \mathrm{Wang}^{2}, \mathrm{D} . \mathrm{Cai}^{1}$; \\ ${ }^{1}$ Yong Loo Lin School of Medicine, National University \\ of Singapore, Singapore, Singapore, ${ }^{2}$ Institute of Molecular \\ and Cell Biology, Singapore, Singapore
}

Phagocytosis is a specialised mechanism utilised by mammalian cells, particularly the cells of the immune system, such as the dendritic cells (DC) and macrophages, to protect the host against infection. The phagocytosis process also creates intracellular antigen pools essential for the antigen presentation machinery in DC. This process involves a complex cascade of pathways, from the ligation of surface receptors of phagocytes with components of microorganisms surface, formation of phagosomes and subsequently phagolysosomes, to the eventual presentation of foreign antigens. VAMP-3, VAMP-7, and VAMP-8/endobrevin have been shown previously to function in the endocytic pathways. To determine their roles in regulating antigen uptake via phagocytosis in DC, plasmid DNA encoding VAMP-3, VAMP-7, and VAMP-8 were transfected into DC and assayed for their effects on the phagocytic activity. Our results showed that VAMP-7, and VAMP-8 but not VAMP-3 co-localised with LAMP-2. In addition, significant amounts of VAMP-3, VAMP-7 and VAMP-8 were recruited to the phagosomes during bacterial ingestion. Over-expression of VAMP-8 but not VAMP-3 significantly inhibited phagocytosis in DC. Furthermore, the phagocytic activity of VAMP-8-deficient DC was significantly higher than wild-type DC, thus further confirmed that VAMP-8 negatively regulated phagocytosis in immature DC.

\section{P129}

\section{Identification of new endocytic receptors to improve tumor antigen presentation in dendritic cells}

\author{
E. Tagliani ${ }^{l}$, P. Guermonprez ${ }^{2}$, S. Amigorena ${ }^{2}$, F. Benvenuti $^{l}$, \\ O. R. Burrone ${ }^{1}$; \\ ${ }^{1}$ ICGEB, Trieste, Italy, 2U520 INSERM Institut Curie, Paris, \\ France
}

Valuable proofs of concept have been obtained with respect to the capacity of dendritic cells (DCs) to induce tumor specific T-cell responses. The modality of delivery of tumor antigens to DCs is one of the critical factors to improve current DC-based vaccination protocols.

We employed an antibody phage display approach to identify DCs endocytic receptors capable to deliver exogenous antigens to both MHC class I and MHC class II antigen presentation pathways. We isolated a high affinity antibody that recognizes CD36, a multiligand scavenger receptor primarily expressed by the CD $8 \alpha^{+}$ subset of conventional DCs. To assess the ability of CD36 to mediate the uptake, processing and presentation of soluble Ags, we produced a fusion protein containing the full length of Ovalbumin at the $\mathrm{C}$-terminus of the scFv molecule. We show that in vivo delivery of soluble OVA to DCs through CD36 efficiently activate antigen specific $\mathrm{T}$ helper lymphocytes (OT-II) and cytotoxic T lymphocytes (OT-I) endowed with effector functions. Mice immunized with scFv $\alpha \mathrm{CD} 36-\mathrm{OVA}$ develop antigen specific CD8 T cells and anti-OVA antibody response which prevent tumor growth upon challenge. These data define CD36 as a novel receptor to induce cross-presentation by $\mathrm{CD} 8 \alpha^{+}$DCs. In addition, its ability to induce both cytotoxic and humoral immune responses makes it an appealing candidate for the development of immunotherapeutical protocols.

\section{P130}

\section{Influence of Cytidine-phosphate-guanosine oligodeoxynucleotides (CpG-ODN) on maturation of canine bone marrow-derived dendritic cells}

\author{
C. Adam, S. Lange, S. Altmann, A. Sekora, M. Dahlhaus, \\ M. Freund, C. Junghanss; \\ University of Rostock, Rostock, Germany
}

Dendritic cells (DC) play an important role in allogeneic immune responses. The canine hematopoietic stem cell transplantation model has been shown to be excellent for development of new transplantation regimen and posttransplantation procedures. Here, we report on the use of CpG-ODN for maturation of canine DC. 
Methods: Canine mononuclear cells were isolated from bone marrow by density gradient centrifugation (density $1.074 \mathrm{~g} / \mathrm{ml}$ ). Cells were cultured in medium supplemented with human GM-CSF and canine IL-4. Different concentrations of CpG-ODN $(2,5,10 \mu \mathrm{g} / \mathrm{ml})$ and TNF $\alpha(10 \mathrm{ng} / \mathrm{ml})$ were added, respectively. Non-adherent cells were removed by medium exchange at days 3 and 5. DC were harvested at day 7 .

Results: Harvested cells showed characteristic morphology of DC. Comparison of cell numbers at days 0 and 7 indicated median DC yields of $38 \%$ in the control, $62 \%$ for TNF $\alpha$ treated cells, and 75 , 72 , and $83 \%$ for cells grown in the presence of 2,5 , and $10 \mu \mathrm{g} / \mathrm{ml}$ CpG-ODN, respectively. Differences were significant between control and the $10 \mu \mathrm{g} / \mathrm{ml} \mathrm{CpG-ODN}$ group. As determined by flow cytometry MHC II and CD11c expression increased ranging from 1.3- to 1.8-fold and 1.8- to 3.1-fold over time, respectively, in all groups.

Conclusion: The use of CpG-ODN for canine DC maturation allows higher cell yields compared to conventional maturation methods. Currently, the ability of canine CpG-ODN generated DC to induce allogeneic $\mathrm{T}$ cell response is tested in vitro and in vivo (CFSE-staining, posttransplantation vaccination experiments).

\section{P131 \\ Shift of dendritic cells from plasmacytoid to myeloid in leukemic plasmacytoid dendritic cell line (PMDC05) established from a patient with CD4 + CD56 + acute leukemia}

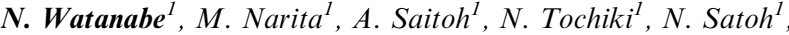 \\ S. Hashimoto ${ }^{2}$, T. Furukawa ${ }^{2}, K$. Toba $^{2}, K$. Toba $^{2}$, K. Toba $^{2}$, \\ K. Toba ${ }^{2}, K$. Toba $^{2}$, I. Fuse ${ }^{2}$, I. Fuse ${ }^{2}$, Y. Aizawa ${ }^{2}$, M. Takahashi ${ }^{1}$; \\ ${ }^{1}$ Laboratory of Hematology and Oncology, Graduate School \\ of Health Sciences, Niigata University, Niigata, Japan, \\ ${ }^{2}$ Department of Hematology, Graduate School of Medical \\ and Dental Sciences, Niigata University, Niigata, Japan
}

We established a leukemic plasmacytoid dendritic cell (pDC) line (PMDC05) from leukemia cells of a patient with CD4 + CD56 + acute leukemia. PMDC05 showed a complex chromosomal abnormalities (44, XY) including $\operatorname{add}(5)(\mathrm{q} 22)$, $\operatorname{add}(15)(\mathrm{q} 26)$ and $\operatorname{del}(15)(\mathrm{q} 11 \mathrm{q} 15)$, which were reported as the frequent aberrations in CD4 + CD56 + pDC leukemia/lymphoma. PMDC05, which morphology was similar to plasma cells, was positive for CD4, CD56, CD33, CD86, HLA-DR, and CD40 but negative for linage markers. Cytokine receptors for GM-CSF, IL3Ra (CD123) and IL-6Ra were positive on PMDC05. The expression of Trail and Flt-3L was positive. By the culture with IL-3, CpG-A/B, GM-CSF, molecules associated with antigen presentation such as CD1a and CD40 were upregulated. Besides, the addition of LPS increased the expression of CD40, CD80 and CD83 on PMDC05. PMDC05 by itself possessed a potent antigen presenting ability to naïve $T$ cells and the treatment of PMDC05 with IL-3, CpG-A/B, or GM-CSF enhanced the antigen presenting ability. TLR7, TLR 8 and TLR 9 as well as TLR1, TLR2, TLR4 were demonstrated to be expressed on PMDC05 by RT-PCR and RQ-PCR showed that the expression of TLR7 and TLR9 was most characteristic. lambdalike 14.1 and pre Ta was also demonstrated to be expressed on PMDC05 by RT/RQ-PCR. By culture of PMDC05 with IL-3, the major population of PMDC05 with CD123+/BDCA1(pDC) shifted to the population with CD123-/BDCA1 + (myeloid DC). These data demonstrated that newly established PMDC05 is involved in pDC lineage and a differentiation from plasmacytoid to myeloid could be plausible in DC lineage.
P132

Highly efficient antigen targeting to murine plasmacytoid dendritic cells (PDC) using mPDCA-1specific antibody conjugates: $P D C$ activation dependent priming of naive $\mathrm{CD} 4+\mathrm{T}$ cells

\section{J. A. A. Fischer, J. Schmitz, A. Dzionek; Miltenyi Biotec, Bergisch Gladbach, Germany}

PDC represent a distinct subset of dendritic cells characterised by their plasmacytoid morphology and the ability to produce large amounts of IFN-alpha in response to viruses and microbial compounds. It is still controversial to which degree PDC are capable of taking up exogenous antigens and play a role in priming of naïve $\mathrm{T}$ cells.

Here we investigated the function of the recently described PDC marker mPDCA-1 as an antigen-uptake receptor. Using a monoclonal antibody (mAb) against mPDCA-1 we were able to specifically target PDC both in vivo and in vitro. Ligation of mPDCA-1 leads to a rapid internalisation of the antibodyreceptor complex, indicating that mPDCA-1 might serve as a PDC-specific antigen-uptake receptor. Furthermore, targeting PDC with OVA-conjugated anti-mPDCA-1 mAb, but not with an equivalent amount of soluble OVA, or OVA conjugated to an isotype control $\mathrm{mAb}$ resulted in efficient proliferation of naive OVA-specific CD4 $+\mathrm{T}$ cell receptor transgenic $\mathrm{T}$ cells in vitro. However, this was clearly dependent on $\mathrm{CpG}$ oligonucleotide activation of PDC, since non-activated PDC were not able to prime OVA-specific $T$ cells. Our results indicate that efficient processing and presentation of antigen by PDC for stimulation of naïve $\mathrm{CD} 4+\mathrm{T}$ cells requires appropriate $\mathrm{PDC}$ stimulation. Currently we investigate, whether PDC may further contribute to adaptive immunity by cross-presenting antigens taken up via mPDCA-1 to naïve CD8 $+\mathrm{T}$ cell receptor transgenic $\mathrm{T}$ cells.

\section{P133}

\section{CD11c provides a uniquely effective immunotarget} for the generation of $\mathbf{T}$ and $\mathbf{B}$ cell responses in vivo

\section{A. L. White, F. Castro, A. Tutt, R. French, M. Glennie; Tenovus Research Laboratory, Southampton, UK}

The absolute and relative magnitude of B cell and CD4/CD8 T cell responses generated when antigen is targeted to dendritic cell (DC) receptors in vivo appears to depend in large part upon whether $\mathrm{CD} 8+$ or $\mathrm{CD} 8-\mathrm{DC}$ subsets are targeted. The CD11c/CD18 dimer is expressed at high levels on both CD8 + and CD8- DCs. In this study, we compared the benefits of targeting antigen to CD11c versus other APC surface molecules using antigen chemically conjugated to Fab' fragments of $\mathrm{mAb}$ specific for different receptors. Initial investigations showed that inoculation with [anti$\mathrm{CD} 11 \mathrm{cxOva}$ ] generated between 1.5 and 6-fold greater proliferation of adoptively transferred Ova-specific CD4 T cells, and fivefold greater proliferation of Ova-specific CD8 $\mathrm{T}$ cells than [antiDec205xOva] or [anti-MHCIIxOva]. However the major benefit of $\mathrm{CD} 11 \mathrm{c}$ targeting was seen in humoral responses. A single inoculation with $100 \mathrm{ng}$ of Ova coupled to anti-CD11c produced anti-Ova antibody titres of $1: 10,000$ by D7 without adjuvant. Similar titres were obtained with [anti-CD18xOva] or [anti-MHCIIxOva], but only with 5- and 25-fold higher doses of antigen, respectively. In contrast, targeting to Dec205 (on CD8 + DCs) or CD11b (another partner of CD18 on CD8- DCs) produced little or no antibody response. Immunisation of mice with immunoglobulin idiotype from the $\mathrm{B}$ cell lymphoma, $\mathrm{BCL}_{1}$, coupled to anti-CD11c provided 
protection from subsequent challenge with a large dose of tumour $\left(10^{7}\right.$ cells). Thus, targeting of antigen to CD11c is uniquely effective in the generation of both cellular and humoral immunity and may provide an efficacious vaccination protocol for protection against tumour growth.

\section{P134 \\ Phenotype,ultrastructure and function of hemophagocytic dendritic cells associated with natural killer dysfunction}

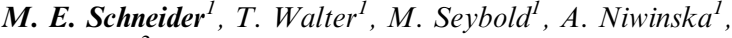 \\ P. Walther ${ }^{2}$; \\ ${ }^{1}$ Sektion Experimentelle Anaesthesiologie, Ulm, Germany, \\ ${ }^{2}$ Sektion Elektronenmikroskopie, Ulm, Germany
}

Introduction: NK deficiency is a hallmark of life threatening hemophagocytic lymphohistiocytosis (HLH). HLH manifests early in childhood, cells of the $\mathrm{Mo} / \mathrm{DC}$ lineage massively phagocytose hematopoetic cells but spare lymphocytes. Mutations in granule exocytosis molecules such as Perforin, Munc134, Rab27a and Syntaxin 11 have been demonstrated to be involved. In addition other immune deficiencies including sepsis may develop hemophagocytic syndromes but remain often unrecognized. We aimed at the description of phagocytic DC phenotypes in various hemophagocytic syndromes of genetic as well as acquired origin.

Methods: Phagocytic DC were enriched by cell cultures from blood or bone marrow within 28 days. Immune phenotypes, cytokine release and ultrastructural properties were compared to phagocytic dendrititc cells prepared from healthy donors using magnetic beads.

Results: Phagocytic DC from genetic HLH often repopulated from precursors co-expressing CD2. Surface antigen expression of the mature hemophagocytic DC was similar between genetic and acquired cases in that TLR2, CD86, CD40, were strongly expressed, but TLR3, CCR7, CD80 were negative. Ultrastructurally, the hemophagocytic cells had prominent intracellular vacuoles of engulfed cytoplasm. Fusion of individual vesicles was not observed but instead some vesicles had double and triple outer membrane layers.

Conclusion: Diseases characterized by high IFN-g due to severe virus infections and either genetic or acquired NK cell dysfunction induce a massive expansion of hemophagocytic DC lacking antigen presenting function, TLR3, CCR7, and TLR3 expression. Dysregulated phagocytosis and digestion of phagocytosed material appears to be due to impaired vesicle fusion events.

\section{P135 \\ Different DC populations preferentially interact with naïve, memory, and effector $\mathbf{T}$ cells, or with regulatory $\mathbf{T}$ cells}

\section{R. Muthuswamy, T. Reinhart, P. Kalinski; \\ University of Pittsburgh Cancer Institute, Pittsburgh, PA, USA}

Effective anti-cancer responses are counteracted by $\mathrm{FoxP}^{+}{ }^{+}$regulatory $\mathrm{T}$ cells. In attempt to develop improved DC-based cancer vaccines, able of preferentially inducing the effector-type (Teff), rather than regulatory-type (Treg) immune responses, we have analyzed the ability of different DC types to secrete the chemokines preferentially attracting naïve and central memory cells (CCL19),
Teff-cells (CCL5, CXCL9, CXCL10, and CXCL11), or Treg cells (CCL22 and CXCL12). We observed that the frequently-used in clinical trials "standard" DCs (sDC; induced by the combination of IL- $1 \beta / \mathrm{TNF} \alpha / \mathrm{IL}-6 / \mathrm{PGE}_{2}$ ), preferentially produce Treg-attracting CCL22 (and lesser amounts of CXCL12), but only marginal levels of the Teff-attracting chemokines, or CCL19. In sharp contrast, type-1 polarized DCs $(\alpha \mathrm{DC} 1 \mathrm{~s}$; induced by the combination of IL$1 \beta / \mathrm{TNF} \alpha / \mathrm{IFN} \alpha / \mathrm{IFN} \gamma / \mathrm{p}-\mathrm{I}: \mathrm{C})$, preferentially produced CCL19 and the Teff-attracting chemokines, but showed only marginal production of the Treg-attracting CCL22 and CXCL12. In accordance with these distinct chemokine production patterns, $\alpha \mathrm{DC} 1 \mathrm{~s}$ preferentially attracted naïve, memory, and effector T cells, while sDCs showed elevated ability to attract FoxP3 ${ }^{+} \mathrm{T}$ cells. The currentlydemonstrated preferential interaction of different types of DC with either naïve/memory and effector $\mathrm{T}$ cells or with Tregs opens the possibility of improving the clinical outcomes of cancer vaccination, by selectively activating the desirable types of immune cells.

\section{P136}

How inflaming the skin affects the lymph node: Phenotype of dendritic cells and cytokine response to topical CpG adjuvant

\author{
H. M. Najar, J. P. Dutz; \\ $C F$ Research Institute of Children and Women's Health, \\ Vancouver, BC, Canada
}

Topical administration of oligodinucleotide CpG, a Toll like receptor-9 (TLR-9) agonist, improves the immune response to subcutaneous protein antigen; enabling antigen-specific cytotoxic $\mathrm{T}$ lymphocyte priming. This provides a simple method to improve vaccine responses. The primary target for antigen and adjuvant are thought to be skin dendritic cells (DC). However, it is unclear which DC subset is responsible for the priming of $\mathrm{T}$ cells to subcutaneous antigen and how topical TLR-9 agonist affects this DC subset. We immunized the $\mathrm{C} 57 \mathrm{Bl} / 6$ mice with Fluorescinated ovalbumin (OVA-FITC) subcutaneously and applied CPG onto the overlying skin. Analysis of the draining lymph nodes at $24 \mathrm{~h}$ demonstrated that $\mathrm{CD} 11 \mathrm{c}^{\text {high }}, \mathrm{CD} 207^{\text {low }}, \mathrm{CD} 8^{\text {low }} \mathrm{DC}$ residing in draining lymph node (DLN) predominantly acquire the protein antigen. Topically FITC labeled LC appeared within the LN at $24 \mathrm{~h}$. The data shows that LC's do not acquire the subcutaneous antigen but $\mathrm{CpG}$ enhances their migration to the LN. It also indicates that all CD207 ${ }^{+}$ and CD207 ${ }^{-}$DC's show CD86 up-regulation as soon as $24 \mathrm{~h}$ after topical $\mathrm{CpG}$ administration. This provides evidence that microenvironment provided by $\mathrm{CpG}$, and through TLR9 stimulation, causes multiple DC populations in the skin to become activated. Further, topical $\mathrm{CpG}$ induces expression of type-1 interferons in the local skin and muscle as determined by MX staining. In contrast to parenteral $\mathrm{CpG}$ administration, no systemic cytokine release is noted following topical $\mathrm{CpG}$ administration. These results favor both a LC-dependent and an LC-independent mechanism of local inflammation in the adjuvant activity of topical $\mathrm{CpG}$.

\section{P137}

Development of Provenge(r) (sipuleucel-T), an active cellular immunotherapy for prostate cancer

\section{L. Urdal; \\ Dendreon Corporation, Seattle, WA, USA}

Provenge ${ }^{\circledR}$ (sipuleucel-T) is an investigational active cellular immunotherapy for prostate cancer. It is composed of autologous peripheral blood antigen presenting cells (APC) pulsed with 
PA2024, a recombinant Prostatic Acid Phosphatase (PAP)-cytokine fusion protein and is designed to stimulate an immune response to the patient's tumor.

Results from a double blind, placebo-controlled phase 3 trial (D9901) showed that asymptomatic patients with metastatic, androgen-independent prostate cancer, who received Provenge, had a median survival of 25.9 months compared to 21.4 months for patients in the placebo arm, a 4.5 month improvement ( $P$-value $=0.01$, hazard ratio $=1.7)$. In addition, 34 percent of patients who received Provenge were alive at 36 months compared to $11 \%$ of patients who received placebo. None of the patients were lost to follow-up for survival. Provenge was well tolerated with the most common adverse events reported being fever and chills lasting for 1-2 days.

An analysis of subsequent therapy men received after their disease had progressed indicated that there were no imbalances between the two treatment arms of the trial with regard to subsequent use of chemotherapy based regimens. In addition Cox regression multivariate analysis further substantiated the validity of the survival benefit seen in the studies.

The development history, clinical trial results and regulatory milestones will be discussed.

\section{P138}

\section{Dendritic cell cytotoxicity against tumor cells via a NO-dependent mechanism : implications for immunotherapy}

\section{A. Nicolas, J. Fraszczak, D. Cathelin, B. Bonnotte, INSERM U866, Dijon, France}

Dendritic cells play an essential role in anti-tumor immune response as they are powerful antigen-presenting cells for activation of specific antitumor $\mathrm{T}$ lymphocytes. Furthermore, dendritic cells may, after activation by IFN- $\gamma$ or LPS, also act as potent cytotoxic cells against tumor cells. We demonstrated that activated rat bone-marrow-derived dendritic cells (BMDCs) are powerful cytotoxic cells against rat, mouse and human tumor cells. The cytotoxic DCs exhibit mature DC phenotype, produce high amount of IL-12, but they still have their phagocytosis property as immature DCs. The DC cytotoxic activity required cell-cell contact. The lost of membrane integrity, the absence of chromatin condensation of the dead tumor cells suggest a non apoptotic pathway confirmed by the non-involvement of the caspases. Studies of tumor death cell mechanisms showed that DC cytotoxicity does not involve perforin/granzyme or death receptor pathway. We demonstrated that the DC cytotoxicity is mainly mediated by a NO-dependent mechanism. In vivo studies confirmed that LPS induced NO secretion by tumor-infiltrating DCs and a dramatic reduction of the tumor volume. Taken together, our results show that LPS-activated BMDCs are powerful cytotoxic cells against tumor cells and could be a very efficient tool for immunotherapy.

\section{P139 \\ A novel mannose-based antigen delivery system potentiates immunogenicity via specific DC subtypes and toll-like receptor $\mathbf{4}$ functionality}

K. Sheng, M. Kalkanidis, G. A. Pietersz, D. S. Pouniotis, S. Esparon, C. Tang, V. Apostolopoulos;

Burnet Institute, Melbourne, Australia
Antigen mannosylation is an evident approach to potentiate antigen immunogenicity, due to the enhanced antigen uptake and presentation by antigen presenting cells. To overcome several disadvantages associated with conventional methods for antigen mannosylation, we have, through chemical synthesis, developed a novel mannose-based antigen delivery system utilizing a polyamidoamine (PAMAM) dendrimer to optimize antigenicity. It is demonstrated that mannosylated dendrimer ovalbumin (MDO) is a potent immune inducer. With a strong binding avidity to dendritic cells (DCs), MDO potently induced OVA-specific $T$ cell proliferation in vitro due to enhanced antigen presentation and induction of DC maturation. Mice immunized with MDO generated strong OVA-specific CD4+/CD8+ $\mathrm{T}$ cell and antibody responses. MDO targeted lymph node DCs to cross-present OVA, leading to OTI CD8 $+\mathrm{T}$ cell proliferation. Upon challenge with B16-OVA melanoma, mice preimmunized with MDO either did not grow tumors or displayed a much more delayed onset, slower kinetics of tumor growth than that of OVA-immunized mice. Subsequent mechanism studies indicated that MDO differentially activated DC subsets derived from defined cytokine cultures. Surprisingly, in contrast to the previous study, MDO induced the cross-presentation capability in CD8-, rather than CD8 +, equivalent Flt3-L DCs. Moreover, DC maturation and its capacity in antigen processing and presentation were largely dependent on toll-like receptor 4 functionality, based on the $\mathrm{C} 3 \mathrm{H} / \mathrm{HeJ}$ and TLR 4 blocking antibody models. For the first time, the immunogenicity of a mannosebased antigen delivery system was studied in detail. With its exceptional adjuvanticity, mannosylated dendrimer has a significant immunotherapeutic value.

\section{P140}

\section{Dendritic cell like function of ER-TR9 ${ }^{+}$marginal zone macrophages}

M. yszkiewicz, N. Ziętara, M. Rodhe, K. Dittmar, J. Jabońska, S. Weiss;

Helmholtz Centre for Infection Research, Braunschweig, Germany

ER-RT9 $^{+}$marginal zone macrophages (MZM)) are found in the spleen at a strategic position for capturing blood borne antigen and pathogens. They are located where the central arteriole opens into the splenic sinus and form a tight network by extending long dendroid like protrusions. This DC like property prompted us to investigate the antigen presenting capacity of such cells. ER-TR9 ${ }^{+}$MZM were able to present peptide and soluble antigen to naïve CD4 $\mathrm{T}$ cells in vitro. Interestingly, such cells were also able to efficiently present peptide to major histocompatibility complex MHC class I restricted naïve CD8 T cells. More importantly, ER-TR9 ${ }^{+}$MZM cells were able to efficiently processe native OVA and crosspresent it to OT-I T cells in vitro while other splenic macrophages (MФ), like metalophillic $M \Phi$ and red pulp $M \Phi$ were unable to do so. This cross-presenting ability of ER-TR9 ${ }^{+}$ MZM was found for soluble OVA as well as for latex beadassociated OVA. ER-TR9 ${ }^{+}$MZM, in addition to $\mathrm{CD} 8 \alpha^{+}$DC and plasmacytoid DC, are effective in phagocytosis of Listeria monocytogenes that invade the spleen. By ex vivo studies, we could show that both ER-TR9 ${ }^{+}$MZM and CD8 $\alpha^{+}$DC were able to efficiently cross-present antigen secreted by L. monocytogenes to CD8 T cells. Our data identified ER-TR9 ${ }^{+}$MZM as professional splenic APCs with DC like properties. They are able to cross-present antigen and to activate naïve CD4 and CD8 T cells. 


\section{P141}

Intra-tumoral myeloid dendritic cells in $\mathrm{HCC}$ patients are impaired in their function and show monocytic characteristics

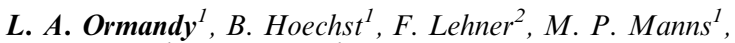 \\ T. F. Greten ${ }^{l}$, F. Korangy ${ }^{I}$; \\ ${ }^{1}$ Department of Gastroenterology, Hepatology and Endocrinology, \\ Hannover Medical School, Germany, ${ }^{2}$ Department of Visceral \\ Surgery, Hannover Medical School, Germany
}

Background and significance: Hepatocellular carcinoma (HCC) is the fifth most common cancer worldwide with a poor prognosis and one for which immunotherapy remains a viable option. A number of studies including our own have shown that tumorspecific humoral and cellular immune responses are detectable in HCC patients despite the progression of the tumor. A detailed characterization of the immune responses at the tumor site of HCC patients is crucial in development of successful immunotherapy of cancer. The aim of this study was to characterize intratumoral myeloid dendritic cells (mDCs) in patients with hepatocellular carcinoma.

Methods: CD1c ${ }^{+}$myeloid DCs were directly isolated from tumors, malignant ascites and peripheral blood of HCC patients. Cell surface markers (CD80, CD86, CD83, CD14, HLA-DR and CCR7) on myeloid DCs were analyzed by FACS. Cytokine secretion was measured by Intracellular cytokine staining. The cells were also FACS sorted and used in an allogeneic mixed lymphocyte reaction.

Results: Intra-tumoral myeloid DCs and mDCs from ascites showed an immature phenotype with lower expression of costimultary molecules (CD80, CD86, CD83, HLADR) that fail to upregulate upon LPS stimulation. Furthermore, intra-tumoral DCs secrete decreased amounts of IL-12 and no TNF- $\alpha$. The CD14 expression on mDCs from tumor of HCC patients shows that these cells have more characteristic of monocytes than dendritic cells.

Conclusions: Our results show that myeloid DCs from the tumor and ascites of HCC patients are impaired in their function and differ from the myeloid DCs in peripheral blood of these patients. These cells appear to be more monocytic and therefore can hamper anti-tumor immune responses at the tumor site in HCC patients.

\section{P142 \\ Differentiation and characterization of dendritic cells from HOXB4 transduced haematopoietic stem cells}

\section{A. M. Baru, M. Eder, M. Scherr, R. E. Schmidt, G. M. Behrens; Medizinische Hochschule Hannover, Hannover, Germany}

Dendritic cell based vaccination, especially in immunity against cancer, has drawn tremendous attraction over the past decade. Although an attractive approach, it suffers a major constraint in obtaining feasible number of autologous/allogenic cells to be available for transplantation. While in vitro expansion and differentiation of haematopoietic stem cells (HSC) can be foreseen as most viable alternative, the complexity to expand HSC in vitro poses a significant limitation for its clinical application.
Ectopic expression of HOXB4 in purified HSCs has been shown to enhance the ability of HSCs to regenerate about 1,000 folds higher without losing their stem cell capacity, impairment in normal differentiation or undergoing transformation. Thus we aimed to differentiate murine HSCs transduced with HOXB4, into dendritic cells and confirm this differentiation functionally. We standardized in vitro culture conditions required to differentiate HOXB4 transduced HSC into dendritic cells. After about 10-11 days of culture most of the living cells adhered lightly to plastic and showed dendritic morphology. These cells were confirmed to be dendritic cells by their microscopic appearance and expression of canonical phenotypic surface markers. These dendritic cells also underwent maturation on TLR stimulation as scored by upregulation of costimulatory molecules. The functional competency of these differentiated dendritic cells was confirmed by uptake assay using fluorescent labelled beads and by in vitro $\mathrm{T}$ cell stimulation assays using OVA transgenic $\mathrm{T}$ cells.

The in vitro differentiation of HOXB4 transduced HSC appears to be highly promising in the field of dendritic cell vaccination.

\section{Clinical Development}

\section{P143 \\ Improvement of clinical grade maturation of monocyte derived dendritic cells}

A. Ten Brinke, M. L. Karsten, M. C. Dieker, M. M. Meyering, J. J. Zwaginga, S. M. van Ham;

Sanquin Research, Amsterdam, The Netherlands

Ex vivo generation of autologous antigen-loaded DCs followed by patient vaccination with these DCs for the in vivo generation or boosting of antigen-specific $\mathrm{T}$ cell mediated immunity is currently studied in clinical trials.

Ex vivo, immature DCs can be generated from monocytes and subsequently differentiated into mature DCs by various cocktails of maturation factors. Clinical studies require protocols where a sufficient number of well characterized highly immunogenic DCs are produced according to current good manufacturing practice (cGMP) Guidelines. In addition, for antitumour immunotherapy, the ex vivo generated DCs should combine a few important characteristics in order to induce the tumour-specific CTLs. The DCs should (1) be capable to migrate to lymphoid organs, (2) induce Ag-specific T cell activation and (3) produce the polarisating cytokines (mainly IL-12) that will induce Th1 responses.

A common used $\mathrm{PGE}_{2}$-based maturation cocktail in clinical trials induces very good maturation, but no IL- 12 p70 production. Other maturation cocktails, which include TLR ligands, induce IL- 12 p70 production, but render these DCs less migratory. Thus, IL-12 production and migration by DCs seem to be inversely correlated processes. However Mailliard et al. (Cancer Research, 2004) have found a cocktail which induces DCs which can both migrate and produce IL-12, but because of its 5 components this cocktail is quite expensive.

Here, we report a cost-effective, new clinical grade maturation cocktail, consisting of MPLA and IFN $\gamma$, which fulfils the requirements for induction of type 1 immunity. Matured DCs combine migration with very high IL-12 production upon CD40L stimulation. 


\section{P144}

\section{Counterflow elutriation (Elutra ${ }^{\mathrm{TM}}$ ): a critical step towards clinical-grade closed system production of dendritic cells}

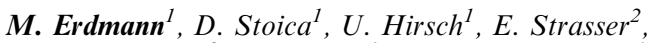 \\ M. Hendelmeier ${ }^{2}$, G. Schuler ${ }^{1}$, B. Schuler-Thurner ${ }^{1}$; \\ ${ }^{1}$ Department of Dermatology, Erlangen, Germany, \\ ${ }^{2}$ Department of Transfusion Medicine and Hemostaseology, \\ Erlangen, Germany
}

Dendritic cell vaccination is a promising option in malignoma therapy. We and other groups successfully induced tumor-specific T-cells in patients by Dendritic Cell immunotherapy resulting in clinical responses.

A major obstacle to a clinical-grade application of this approach is the requirement of clean room conditions in order to generate large amounts of dendritic cells in GMP quality. Therefore great efforts were made to generate a closed system.

We recently demonstrated that counterflow elutriation (CE) performed with autologous plasma elutriation/cultivation medium (RPMI) is feasible. Subsequent direct cultivation within in the same medium renders opening the system for medium exchange unnecessary and is an essential step towards a functionally closed system. Additionally we showed that a higher yield per leukapheresis can be achieved by CE compared to previous approaches. So far we treated over 20 patients with CE-generated Dendritic Cells without observing any in vitro cellular, functional or in vivo clinical difference compared to our previous adherence-generated vaccine.

Here we demonstrate that CE performed in CellGro ${ }^{\circledR}$ and $\mathrm{X}$-vivo ${ }^{\circledR}$ medium yields phenotypical and functional Dendritic Cells.

We therefore propose that CE is an essential step towards a closed system for large-scale Dendritic Cell based immunotherapy.

\section{P145}

\section{A core facility dedicated to transfer of research protocols towards clinical applications}

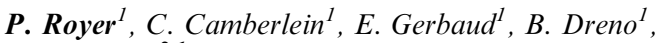
M. Gregoire ${ }^{2,1}$;

${ }^{1}$ Biotherapy CIC, Nantes, France, ${ }^{2}$ Inserm, U 601, Nantes, France

Cellular therapy protocols have to guarantee rigorous criteria concerning the cellular product definition and quality. Those criteria, required by national health agencies, refer to specifications that are generally not considered by experimental studies. Indeed, clinical grade productions need strict culture conditions with the use of good manufacturing practice (GMP) reagents (culture media, cytokines, etc.), according to production process validated and well defined. Unfortunately, this step of protocol optimisation is usually underrated and minimized due to limited interest for research needs.

Thus, within the context of the set up and development of clinical protocols, technological transfer step is essential in order to adapt cellular product from research to clinical constraints. We present here a core facility, named "Plate-forme DTC" (Développement et Transfert Clinique), based in Nantes Hospital and dedicated to transfer of cellular therapy research protocols towards clinical applications. This core facility, integrated within the Nantes Biotherapy Clinical Investigation Centre, was inaugurated in May 2007. It provides technical and technologi- cal supports within a context of tracability and quality insurance.

P146

Toll-like receptor 3 and 7/8 ligands combined with prostaglandin $\mathbf{E}_{2}$ results in high interleukin-12 production and dendritic cell migration

A. I. C. Boullart ${ }^{1,2}$, E. H. J. G. Aarntzen ${ }^{1,2}$, P. Verdijk ${ }^{1}$, J. F. M. Jacobs ${ }^{l}$, D. H. Schuurhuis ${ }^{l}$, D. Benitez-Ribas ${ }^{l}$, M. W. M. M. Van de Rakt ${ }^{l}$, N. M. Scharenborg ${ }^{l}, A$. De Boer ${ }^{1}$, M. Kramer ${ }^{1}$, C. G. Figdor ${ }^{1}$, C. J. A. Punt ${ }^{2}$, G. J. A. Adema ${ }^{1}$, I. J. M. De Vries ${ }^{I}$;

${ }^{1}$ NCMLS, Nijmegen, The Netherlands, ${ }^{2}$ Radboud University, Nijmegen, The Netherlands

Dendritic cells (DC) are professional antigen presenting cells of the immune system that play a key role in regulating $\mathrm{T}$ cell-based immunity. In vivo, the capacity of DC to activate $T$ cells depends on their ability to migrate to the $\mathrm{T}$ cell areas of lymph nodes as well as on their maturation state. Furthermore, their cytokinesecreting profile, especially IL-12p70, is important for skewing the response towards a $\mathrm{T}$ helper 1 type. Here we investigate the effects of Toll like receptor (TLR)-mediated maturation on ex vivo generated human monocytes-derived DC. We demonstrate that in contrast to cytokine-matured DC, TLR ligandmatured DC were able to produce high amounts of IL-12p70, but exhibit a reduced migratory capacity. The addition of prostaglandin $\mathrm{E}_{2}\left(\mathrm{PGE}_{2}\right)$ to the maturation factors poly I:C (TLR3 ligand) and R848 (TLR 7/8 ligand) improved the migratory capacity of TLR ligand-matured DC without affecting their IL-12p70 production upon secondary stimulation. Subsequently, TLR ligand matured DC, whether or not in the presence of $\mathrm{PGE}_{2}$, induced the secretion of a profound Th1 cytokine profile in co-culture with PBL. Based on the here presented data and the availability of the maturation factors for clinical use, we propose a novel clinical grade maturation protocol for ex vivo generated DC. The combination of TLR ligands and $\mathrm{PGE}_{2}$ results in clinical grade, fully mature and stable DC that have an excellent migratory capacity and maintained IL- $12 \mathrm{p} 70$ producing capacity upon T-cell encounter, this results in the secretion of a profound Th1 profile of cytokines.

\section{Future Vaccination Concepts}

\section{P147}

Future preventive and curative vaccination concepts: personal subunit vaccine based plant origin HSP70 and patient's urine derived antigens

\author{
T. Jaliashvili ${ }^{1}, F$. Cellini ${ }^{2}$, R. Iannacone $^{2}, F$. Bruno $^{1}$; \\ ${ }^{1}$ BRT-France Ltd, Dreux, France, ${ }^{2}$ Agro-Biotechnology $R \& D$ \\ Company, Metapontum Agrobios, Metaponto, Italy
}

Analysis of trends in diagnostics [1], vaccination [2,3] and spectacular just action of General Electric Healthcare (GE HC) [4] has pointed out drift towards:

A) Functional diagnostics, wherein dominant will blood and urine proteomics;

B) Personalization as curative so preventive medicines, to what suits virtuously: 
B.1) dendritic cells vaccine for use curative purposes generally;

B.2) subunit vaccine composed from peptide antigens and immunestimulant for use at early phase of diseases preferably.

And maximal efficiency of dendritic cell and subunit vaccines might arrange with:

- Heat stress proteins (HSP), e.g.: HSP70, HSP90 both with strong immune-stimulant and peptide antigens carrier capabilities, since they bind with high affinity to surface receptors of dendritic cells and induces its maturation.

- Patient urine derived peptide antigens, which maintain precise specificity to causative agents of diseases, nevertheless germs mutation or diversity of cancerous cells.We shall represent new opportunity of affordable plant origin HSP70 use for maturation and/or precise priming of dendritic cells vaccine.

1. Baker M (2006) New-wave diagnostics. Nat Biotechnol 24(N):931-938

2. Ulmer JB, Valley U, Rappuoli R (2006) Vaccine manufacturing: challenges and solutions. Nat Biotechnol 11(N11):1377-1383.

3. Berzofsky JA, Terabe M, Oh SK, Belyakov IM, Ahlers JD, Janik JE, Morris JC (2004) Progress on new vaccine strategies for the immunotheraphy and prevention of cancer. J Clin Invest 113(N11):1515-1525

4. Abbott sold laboratory diagnostics to GE HC for $\$ 8.13$ billion; see: Bio Ingredients January 18, (2007)

\section{P148 \\ Amplification of $\mathrm{CD} 4+\mathrm{T}$ cell responses by MHC-loading Enhancer (MLE)}

\section{S. Höpner, K. Dickhaut, J. Eckhard, K. Falk, \\ O. Rötzschke, \\ MDC-Berlin, Berlin, Germany}

In previous studies we identified a set of small organic compounds which have the capacity to enhance loading of peptides/ proteins onto MHCII molecules. The effect of these 'MHCloading enhancer' (MLE) was evident on soluble as well as on membrane bound MHCII-Molecule of living cells. In particular adamantane-ethanole (AdEtOH) strongly accelerates the antigen-loading rate. The catalytic activity of this MLE compound was restricted to HLA-DR molecules expressing Glycine at position $\beta 86$ of the conserved pocket $\mathrm{P} 1$. The residue at this dimorphic position (Glycine/Valine) determines the depth of the pocket P1 and MLE compounds transiently occupying P1 stabilize a receptive conformation, which promotes rapid ligand exchange.

On bone-marrow derived dendritic cells (DC) the presence of MLE compounds mediates effective loading of cell surface MHCII with $\mathrm{T}$ cell antigens. In vitro this effect translates into shifts of the dose/response curves by almost two orders of magnitude when used to challenge CD4 $+\mathrm{T}$ cells. Most importantly, however, there is clear evidence that MLE compounds like AdEtOH act as molecular tools to enhance immune reactions in vivo. In mouse tumour models we observed strongly enhanced $\mathrm{CD}^{+}{ }^{+}$- T-cell responses specific for tumour associated antigens (TAA) when using MLE compounds as vaccine additive ("adjuvant"). However the observation that organic compounds trigger rapid ligand exchange from outside in an allele selective way suggests that 'natural' MLE compounds may represent risk factor for allergy or autoimmune diseases. Preliminary data show that the presence of AdEtOH increases the severity of EAE in strains with MLE-susceptible MHC molecules.
P149

Bisphosphonate-activated human gamma delta $\mathbf{T}$ cells are potent antigen-presenting cells for the induction of Epstein-Barr-Virus (EBV)-specific cytolytic CD8 $+\mathrm{T}$ cells

\author{
S. Landmeier ${ }^{1}$, B. Altvater ${ }^{1}$, S. Pscherer ${ }^{1}$, L. Varnholt $^{1}$, \\ A. Hansmeier ${ }^{1}$, C. Bollard ${ }^{2}$, H. Juergens ${ }^{1}$, C. Rossig ${ }^{1}$; \\ ${ }^{1}$ Pediatric Hematology/Oncology, University Children's Hospital, \\ Münster, Germany, ${ }^{2}$ Center for Cell and Gene Therapy, \\ Baylor College of Medicine, Houston, TX, USA
}

Antigen presentation is an important prerequisite for the induction of $\mathrm{T}$ cell mediated immunity. Activated human $\gamma \delta \mathrm{T}$ cells were recently shown to efficiently present allogeneic and antimicrobial antigens. We extended these observations by evaluating the potential of $\gamma \delta$ T cells to present EBV-derived peptides and induce expansion of fully functional, virus-specific cytotoxic $\alpha \beta$ T cells.

Peripheral blood-derived $\gamma \delta$ T cells were expanded by stimulation with the aminobisphosphonate zoledronic acid for 9 days. Activated $\gamma \delta$ T cells acquired a phenotype characteristic for APC, including upregulation of HLA-DR as well as various costimulatory ligands. The APC function of activated $\gamma \delta$ T cells was tested in vitro using model peptides derived from the highly stimulatory EBV lytic cycle antigen BZLF1 (RAK; HLA-B8), and the low immunogenicity latency-associated antigen LMP2a (FLY; HLAA2). Coincubation of autologous peripheral blood lymphocytes with positively selected $(>90 \%)$ activated $\gamma \delta \mathrm{T}$ cells pulsed with RAK or FLY-peptide for 10 days resulted in selective expansion of pentamer-specific CD3 $+\mathrm{CD} 8+\mathrm{T}$ cells. The percentage of pentamer-reactive $\mathrm{T}$ cells was donor-dependent and slightly inferior to that obtained with peptide-loaded autologous mDCs. Both RAK and FLY-specific CD8 + T cells functionally interacted with peptide-loaded target cells, as demonstrated by specific lysis and IFN- $\gamma$ secretion, comparable to those generated in the presence of mDCs.

In conclusion, we show that bisphosphonate-activated $\gamma \delta \mathrm{T}$ cells have potent immunostimulatory capacity and may thus represent a novel source of easily available and highly efficient APCs for antigen-specific immunity of viral and malignant disease.

\section{P150}

\section{Activation of NF-(kappa)B: a novel approach to generating mature dendritic cells in vivo for vaccination}

\author{
J. Wales ${ }^{1}$, E. Andreakos ${ }^{1}$, B. Foxwell ${ }^{1}$, R. Williams ${ }^{1}$, \\ G. Buchan ${ }^{2}$, M. Feldmann ${ }^{1}$; \\ ${ }^{1}$ Kennedy Institute of Rheumatology, Imperial College, \\ London, UK, ${ }^{2}$ University of Otago, Dunedin, New Zealand
}

Providing safe and efficacious vaccination strategies for viral disease and cancer has proved problematic and requires the development of effective vaccine adjuvants. NF- $\kappa$ B Inducing Kinase (NIK) is an intracellular signalling molecule that, when over-expressed, can activate $\mathrm{NF}-\kappa \mathrm{B}$. When over-expressed in dendritic cells it was shown to increase pro-inflammatory (TNF $\alpha$, IL-6) and Th1 (IL-12, IL-15, IL-18) cytokines. Over-expression induced a mature phenotype with dendrite formation and expression of MHCI/II, CD80, CD86 and IL-2R $\alpha$ on the cell-surface.

Therefore it was assessed for its ability to act as a vaccine adjuvant in vivo. Polyethylenimine complexed to a plasmid that 
expressed NIK was delivered subcutaneously to mice. These complexes were shown to increase specific lymphocyte proliferation and IFN $-\gamma$ production $(P<0.05)$ to an Influenza protein antigen. However as NIK was delivered on a separate vector to the antigen it did not induce a protective immune response in an Influenza challenge model. Therefore, NIK was co-expressed with a model antigen by an adenovirus. In vivo, NIK was able to enhance the immune response to the co-expressed antigen and induce the production of a predominantly type- 1 immune response. Indicated by the increased ratio of $\operatorname{IgG} 2 \mathrm{a}$ to $\operatorname{IgG1}$, as well as increased IFN- $\gamma$ production $(P<0.01)$ and CTL responses when compared to recombinant antigen delivered in Freunds adjuvant. We have now developed adenoviruses that express both NIK and an Influenza antigen to evaluate this approach to protect against disease in vivo.

The activation of NF- $\kappa \mathrm{B}$ directly provides a novel and effective vaccine approach to be used to prevent disease.

\section{P151}

\section{Anti-cancer therapy using dendritic cells and apoptotic tumour cells : pre-clinical investigations for clinical trials}

M. M. Grégoire ${ }^{l}, P$. Royer $^{1}$, L. Lévèque $^{1}, P$. Chevallier $^{l},{ }$, Tarte $^{2}$, V. Quillien ${ }^{3}$, S. Saïagh

${ }^{1}$ INSERM U601, Nantes, France, ${ }^{2}$ UPRES, Rennes, France,

${ }^{3}$ Centre Eugène Marquis, Rennes, France, ${ }^{4} U T C G$, Nantes, France

We have reported, in an experimental model, that treatments based on the injections of dendritic cells which had phagocytosed apoptotic bodies derived from tumour cells were particularly effective in the cure of tumour-bearing animals [1]. We now propose a system using processing and presentation of antigenic molecules from antigen-presenting cells (dendritic cells: DC) primed with apoptotic extracts, which offers new opportunities in anti-cancer adjuvant treatment of myeloid acute leukemia.

We first established the technical conditions for purification, characterisation and production of blast tumour cells isolated from fresh blood in patients before chemotherapy and second, the loading and maturation of efficient activated DC. Here, we describe the induction of stressed-apoptotic preparation of the blast cells as sources of tumor antigens. We also detail the protocol method, in the clinical grade conditions, for generation of the mature dendritic cells pulsed with apoptotic tumour cells and production of the vaccine.

All the experimental procedures that we have used were developed with good manufacturing products for clinical applications. We are presently investigating the feasibility of such a "vaccine" for the treatment of acute myeloid leukaemia in remission phase.

[1] Henry F, Boisteau O, Bretaudeau L, Lieubeau B, Meflah K, Grégoire M (1999) Antigen-presenting cells that phagocytose apoptotic tumor-derived cells are potent tumor vaccines. Cancer Res 59(14):3329-3332

\section{P152}

\section{Targeting of anti-viral cytotoxic T-lymphocytes to tumour cells using MHC-I / antibody derivatives}

B. C. R. King, M. J. Glennie;

Southampton University, Southampton, UK
Anti-tumour responses are not always capable of clearing neoplastic cells. Ignorance, evasion, and anergy contribute to tumour escape, and inhibit the production of effective CD8 + effector cells. However, retargeting of cytotoxic $\mathrm{T}$ lymphocytes recognising cognate peptide from a different antigen, for example common viral antigens, could harness a pre-existing potent cytotoxic response. Levels of these cells can be boosted by vaccination with immunostimulatory $\mathrm{mAb}$. Retargeting can then be achieved by use of a bispecific molecule incorporating both an antibody Fab' fragment against tumour antigen, and a MHC I molecule bearing an immuno-dominant viral peptide. In this proof-of-principle study, OT-I cells recognising the Ova peptide SIINFEKL will be retargeted to kill tumour cells expressing human CD20. Using BL21 (DE3) pLysS E. coli cells, we have expressed and purified $\mathrm{H} 2-\mathrm{Kb}$ in complex with beta 2-microglobulin and the SIINFEKL peptide, and shown that this binds specifically to mouse OT-I T cells. Functional assays show that this construct is capable of redirecting cytotoxic activity against $\mathrm{CD} 20+$ tumour cells in vitro. Heterodimers will be formed by chemical conjugation of the MHC I monomer to the Fab arm of the anti-CD20 mAb Rituximab, using a maleimide linkage. To achieve this the MHC I has been expressed as a fusion protein with the hinge region of a mouse IgG, mutated to retain only one of the original three cysteine residues. These bivalent heterodimers will be tested in vitro. In addition, in vivo retargeting assays have been developed, whereby OT-I cells are retargeted to kill various CD20-expressing cells.

\section{P153 \\ DC for cancer vaccination; exploring new strategies for antigen loading}

\author{
K. Birkholz ${ }^{1}$, J. Dörrie ${ }^{1}$, N. Schaft ${ }^{1}$, M. Schwenkert ${ }^{2}$, \\ C. Kellner ${ }^{2}$, G. Fey ${ }^{2}$, G. Schuler ${ }^{I}$; \\ ${ }^{1}$ Department Dermatology, University Hospital Erlangen, \\ Erlangen, Germany, ${ }^{2}$ Chair of Genetics, University \\ of Erlangen-Nuremberg, Erlangen, Germany
}

Although dendritic cell (DC)-based vaccination of tumor patients has shown encouraging responses in melanoma and renal cell carcinoma, the efficiency of DC vaccines needs further improvement. In order to optimize antigen loading of DC, different methods, such as electroporation of defined tumor-antigen RNA or total tumor RNA, phagocytosis of tumor cells, direct peptide loading, or the use of antibody-antigen constructs need to be thoroughly analyzed and compared. As a model tumor antigen we chose MAGE-A3, a cancer-testis antigen expressed in many tumors including malignant melanoma and multiple myeloma. Furthermore, stimulation of $\mathrm{CD}^{+}{ }^{+}$or $\mathrm{CD}^{+} \mathrm{T}$ cells electroporated with TCR recognizing certain MHC-presented MAGE-A3 epitopes was used as a functional read-out for antigen-presentation efficiency. As one possible loading strategy we applied MAGE-A3-DCLAMP RNA. The DCLAMP sequence targets the antigen to lysosomes, and leads to MHC class II presentation of the antigen. Indeed, we saw in preliminary experiments that electroporation of mature $(\mathrm{m}) \mathrm{DC}$ with MAGE-A3-DCLAMP RNA resulted in HLA-DP4 restricted presentation of the MAGEA3 peptide KKLLTQHFVQENYLEY (KKL, aa 243-258) For a different loading strategy, we cloned antibody-antigen constructs, consisting of a single-chain variable fragment $(\mathrm{scFv})$ directed against DEC205, an endocytosis receptor expressed on the surface of DC, genetically linked to different parts of the MAGE-A3 antigen. The MAGE-A3 antigen should be delivered into the DC by receptor-mediated endocytosis, and subsequently presented on MHC class II molecules. We expressed and purified these con- 
structs, and detected them in western blot analysis. Furthermore, these fusion proteins displayed specific binding to $\mathrm{mDC}$ and DEC205-transfected CHO cells.

\section{P154}

Fusion proteins for flexible antigen targeting to dendritic cell surface receptors

\author{
P. van Endert, R. Kratzer; \\ INSERM U580, Paris, France
}

Targeting of protein vaccines to antigen presenting cells is an attractive strategy for eliciting cellular immune responses. However, because of the difficulty of coupling frequently hydrophobic proteins to targeting devices such as antibodies, its potential has been little exploited. We have produced five antigens, including four hydrophobic viral proteins with an interest as vaccines, as fusion proteins that overcome this difficulty. The soluble proteins also comprise protein $\mathrm{G}$ domains for binding to immunoglobulins, and ubiquitin for proteasome targeting in cross-presentation pathways. The fusion proteins form complexes with a variety of targeting antibodies that mediate binding to dendritic cells and internalization into endolysosomal compartments. This feature should render the fusion proteins highly attractive for rapid screening of various cell surface receptors and antibodies as tools for antigen targeting. Using the model antigen ovalbumin, we show that the fusion proteins, when coupled to several antibodies, elicit both CD $4+$ and CD $8+T$ cell responses in vitro and in vivo at least 100 -fold more efficiently than antigen alone, demonstrating the potential of the strategy for vaccination. Preliminary data indicate that modification of the ubiquitin moiety may further enhance the efficiency of $\mathrm{T}$ cell stimulation by the fusion proteins. In ongoing experiments, the potential of the strategy to elicit $T$ cell responses, in vitro and in vivo, against HIV-nef and HCV core, in humanized HLA-A2 + DR1 + mice is under evaluation.

\section{P155 \\ HER cross-reactivity as a new tool for immunotherapy}

\author{
H. Conrad, K. Gebhard, J. Neudorfer, C. Peschel, \\ H. Bernhard; \\ Klinikum rechts der Isar, Munich, Germany
}

Overexpression of both HER2 and HER 3 is highly correlated in breast cancer. The HER2/HER3 heterodimer functions as an oncogenic unit contributing to the malignant phenotype of the tumor. Double targeting of HER2 and HER3 with cross-reactive cytotoxic T lymphocytes (CTL) may therefore be a promising new aspect in immunotherapy.

We generated tumor-reactive HER $2_{369-377}$ specific $T$ cell clones using either stimulation of CD8 + T cells with autologous HLAA2 positive dendritic cells (DC) transfected with HER2-mRNA or stimulation of HLA-A2 negative CD $8+\mathrm{T}$ cells with HLA-A2

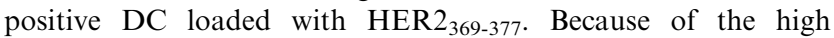
homology within the EGFR-family the resulting CTL clones were tested in lysis assays with $\mathrm{T} 2$ cells loaded with peptides from EGFR, HER3 and HER4 corresponding to HER2 369-377. To investigate the recognition of endogenously processed HER 3 and HER4 we conducted IFN- $\gamma$ ELISA with K562tA2 cells trans- fected with the respective HER plasmids as well as with a HER2 and HER3 expressing cell line treated with siRNA against HER2 and HER3, respectively.

Interestingly, the HER $2_{369-377}$ specific CTL clones were not only able to recognize the synthetic HER 3 and/or HER4 peptides corresponding to HER $2_{369-377}$ but also a cell line expressing either HER2, HER3 or HER4. Using downregulation of the respective HERs we could show that HER2 and HER3 both contribute to the recognition by the CTL clones.

These interesting new findings open new avenues for the development of HER-directed immunotherapies e.g. vaccination with peptides stimulating cross-reactive CTL or adoptive $\mathrm{T}$ cell transfer of cross-reactive CTLs.

\section{P156}

\section{Activation of tumor-specific $T$ cells with DCs expressingNY-ESO-1 after transfection with the lipophosphoramide synthetic vector KLN5}

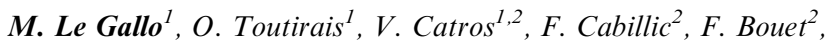

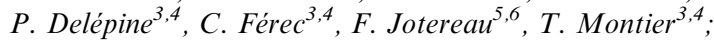 \\ ${ }^{1}$ Université de RENNES I, Rennes, France, ${ }^{2} \mathrm{CHU}$ Pontchaillou, \\ Rennes, France, ${ }^{3}$ INSERM, Unité 613, Brest, France, ${ }^{4}$ Université \\ de Bretagne Occidentale, Brest, France, ${ }^{5}$ INSERM, Unité 601, \\ Nantes, France, ${ }^{6}$ Université de Nantes, Faculté des Sciences, \\ Nantes, France
}

Genetic modification of human dendritic cells (DCs) with tumor associated antigen (TAA) cDNA sequences is a promising strategy for active immunotherapy of cancer. As an alternative to the commonly used viral vectors, we report a nonviral gene transfer method based on the use of the lipophosphoramide transfection reagent KLN-5. Transfection efficiency was first investigated using the GFP reporter gene. Expression level was found proportional to the amount of transfection reagent used. Experimental conditions related to the ratio of KLN5/plasmid DNA and allowing both significant transgene expression and high viability of DCs were then determined. With the established method, the NY-ESO-1 cancer germ line TAA coding gene was found efficiently transfected as shown by tumor antigen mRNA expression analysis. Antigen processing and presentation of the immunodominant (SLLMWITQC157-165) epitope in the HLA-A*0201 context was demonstrated by specific activation of the NY-ESO-1 CD8 + T cell clone. Our data show that transfection of DC using KLN-5 represents a new efficient way to stimulate TAA-specific T cells.

\section{P157}

CD40-activated $B$ cells as antigen presenting cells: High-dose vaccinations are feasible and non-toxic in vivo

\author{
T. Liebig ${ }^{1}$, A. Shimabukuro-Vornhagen ${ }^{1}$, C. Wickenhauser ${ }^{2}$, \\ N. Klein-Gonzalez ${ }^{1}$, L. Gryschok ${ }^{1}, S$. Tawadros ${ }^{1}$, \\ M. von Bergwelt-Baildon ${ }^{l}$; \\ ${ }^{1}$ Molecular Tumorbiology and Tumorimmunology, \\ University of Cologne, Germany, ${ }^{2}$ Institute of Pathology, \\ University of Cologne, Germany
}

Dendritic cells (DC) are a key regulator of adaptive immune responses and are thus used as cellular adjuvant for active immu- 
notherapy. Nevertheless, challenges to this approach have been identified - in particular cell purity and yield required for repetitive vaccinations in clinical trials.

Recently CD40-activated B cells (CD40-B) have been studied as a complementary APC. They can be expanded rapidly from small amounts of $\mathrm{PB}$ at $>95 \%$ purity under GMP-like conditions and efficiently prime and expand naïve $\mathrm{T}$ cells in vitro.

For preclinical in vivo testing prior to the first trial in humans using high-dose, repetitive vaccinations with CD40-B we developed a system to generate murine CD40-B (mCD40-B) from B6 mice. mCD40B cells can be expanded more than sixfold within 14 days with a $>90 \%$ purity. To determine long-term toxicity mice were vaccinated twice weekly for 5 weeks using high-doses $\left(3 \times 10^{6} \approx 1 \times 10^{8} / \mathrm{kg}\right)$ of mCD40-B subcutaneously, intravenously or intraperitoneally. Acute toxicity was assessed by vaccinating mice once with very high doses $\left(1 \times 10^{7} \approx 3.3 \times 10^{8} / \mathrm{kg}\right)$ of mCD40-B. In both models there was no difference in survival, weight and clinical appearance compared to control mice. Histopathological assessment of relevant organs did not reveal any pathologic changes. Furthermore, no signs of autoimmunity or alterations in the B cell compartment were observed.

Based on these finding we are now developing an optimized vaccination algorithm for the induction of effective immune responses in a B16 melanoma model as a next step towards the clinical application of CD40-B cells.

\section{P158}

\section{Lentiviral vector-programmed self-differentiated dendritic cells co-expressing GM-CSF/IL-4 and antigens: One-hit, one-day ex vivo manipulation, robust viability and increased performance.}

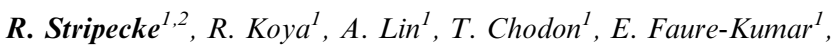 \\ T. Kimura ${ }^{l}$, N. Kasahara ${ }^{1}$, A. Ribas ${ }^{1}$; \\ ${ }^{1} U C L A$, Los Angeles, CA, USA, ${ }^{2}$ Department of Hematology, \\ Medizinische Hochschule Hannover, Hannover, Germany
}

We are developing approaches to improve and facilitate the generation of dendritic cell (DC) vaccines for large-scale trials. We have previously established the "proof-of-concept" that one-hit lentiviral vector transduction of CD14+ purified human monocytes for hGM-CSF and hIL-4 co-expression promoted their self-differentiation into cells with typical DC phenotype and functions. With stable expression of the growth factors, these programmed DCs displayed steady self-maintenance and robust longevity (4 weeks). In analogy, we have demonstrated that DC precursors from mouse bone marrow lentivirally transduced for co-expression of mGM$\mathrm{CSF} / \mathrm{mIL}-4$ underwent autonomous differentiation into DCs. When injected subcutaneously into mice shortly after lentiviral transduction, persistent cellular viability ( $>40$ days) in the injection sites and perceptible dispersion towards the adjacent lymph nodes were observed. Immunohistochemical analyses confirmed the identity of the genetically programmed DCs in the skin and in highly inflamed lymph nodes. The immunostimulatory efficacy of the selfdifferentiated DC vaccines was evaluated with MART1 and TRP2 as lentivirally co-expressed melanoma antigens. C57BL/6 mice vaccinated with programmed DC vaccines showed consistently higher CD8 + antigen-specific responses (measured by cytotoxic T lymphocyte assays, intracellular IFN-g staining and TRP2-specific tetramer staining) than conventionally ex vivo grown DCs. Potent protective and therapeutic anti-tumor effects against B16 melanoma were obtained. Although long-term observation ( $>15$ months) of mice vaccinated with self-differentiated DCs has so far not shown any signs of pathologies, for future clinical studies, we have now validated a safer tricistronic lentiviral design for simultaneous co-expression of HSV-TK/GM-CSF/IL-4 that yields DC self-differentiation and allows their scheduled elimination upon ganciclovir treatment.

\section{P159}

CD40 targeted adenoviral vectors for the in situ targeting of antigens to dendritic cells in human and murine models

\author{
B. N. Hangalapura ${ }^{1}$, P. G. J. T. Wijnands ${ }^{2}$, D. Oosterhoff ${ }^{1}$, \\ A. J. M. van den Eertwegh ${ }^{l}$, D. T. Curiel ${ }^{3}$, A. Pereboev ${ }^{3}$, \\ R. J. Scheper ${ }^{2}$, T. D. de Gruijl ${ }^{1}$; \\ ${ }^{1}$ Department of Medical Oncology, Vrije University Medical \\ Centre, Amsterdam, The Netherlands, ${ }^{2}$ Department of Pathology, \\ Vrije University Medical Centre, Amsterdam, The Netherlands, \\ ${ }^{3}$ Division of Human Gene Therapy, University of Alabama at \\ Birmingham, Birmingham, AL, USA
}

Targeting of tumor antigens to Dendritic cells (DC) has great potential for immunotherapy. Adenoviruses (Ad) are commonly used as vectors for gene thereapy. However, Ad-mediated targeting of DC is difficult as they lack Coxsakie and Adenovirus Receptor (CAR). The interaction of CD40 on DC with its ligand (CD40L) is critical in the generation of protective cell-mediated tumor immunity. To target Ad to DC, we therefore used CFm40L, a bispecific adaptor molecule with the ectodomain of CAR on one end linked to the extracellular domain of mouse CD40L on the other end by a trimerization motif. This adaptor molecule bridges the fiber knob domain of Ad to CD40 on DC. Re-targeting of Ad to $\mathrm{CD} 40$ using $\mathrm{CFm} 40 \mathrm{~L}$ enhances Ad transduction and also induces phenotypic and functional maturation of in vitro differentiated and cultured DC of both murine and human origins. Using CFm40L-targeted Ad encoding MART-1 to transduce human DC in vitro, a superior priming efficiency of specific $\mathrm{CD} 8^{+} \mathrm{T}$ cells was established. These T cells proved to be fully functional, high avidity CTL, lysing MART- $1^{+}$melanoma cells in an HLA-A2 restricted fashion. We are currently studying the in vivo effects of a CFm40L-redirected Ad vaccine in terms of T cell activation and tumor protection in the B16 melanoma model. In order to optimize in vivo delivery for DC targeting, we are also studying CFm40Ldirected Ad transduction in human and murine skin and lymph node samples, tracking transduction, but also phenotypic and functional changes of DC in an interspecies comparative study.

\section{P160 \\ Antigen-pulsed dendritic cells derived exosomes-cell free vaccines against infection

\author{
M. Fajardo-Moser, S. Berzel, C. Hambrecht, J. K. Schnitzer, \\ H. Moll; \\ Institute for Molecular Infection Biology, Würzburg, Germany
}

Dendritic cells (DC) are able to orchestrate the host's immune response to pathogens. The type of $\mathrm{T}$ helper $(\mathrm{TH})$ cells response induced by DC is the result of a complex network of interactions among the different DC subsets, the nature of the microbial and environmental stimuli and the activation status of the DC. In our previous studies we were successful in developing a novel protocol for the generation of adequately activated DC to be used for vaccination. Surprisingly, the IL-12 expression by the immunizing DC was not required for induction of resistance towards L. major. 
We could determine that susceptible mice vaccinated with PFAfixed antigen-pulsed DC are also able to elicit complete and long lasting protection against infection. Furthermore we could determine that adoptively transferred DC migrate to the spleen after vaccination where they are being taken up by resident DC. Those resident DC represent the source of IL-12 required for the induction of protective immunity. In line with these results and in order to develop a cell free vaccine system we are currently investigating the capacity of Ag-pulsed DC derived Exosomes to protect against infection.

\section{P161 \\ Intratumoral injection of dendritic cells loaded with lysate from glioma cells prolongs survival of mice with GL261 gliomas: immunological assessment of the local antitumor effect.}

S. Pellegatta ${ }^{1}$, P. L. Poliani ${ }^{2}$, D. Corno ${ }^{1}$, F. Orzan ${ }^{1}$, E. Stucchi ${ }^{1}$, C. A. Colombo ${ }^{1}$, V. Caldera ${ }^{1}$, S. Musio $^{1}$, G. Finocchiaro ${ }^{1}$;

${ }^{1}$ Istituto Neurologico C. Besta, Milan, Italy,

${ }^{2}$ University of Brescia, Brescia, Italy

Recent work has suggested that the intra-tumoral (it) and peripheral injection of dendritic cells (DC) may prolong survival of patients affected by malignant gliomas (Yamanaka et al. 2005). To evaluate the effect of it injections of DC on survival of mice bearing GL261 gliomas immune-competent mice were injected with GL261 cells and treated with DC loaded with a GL261-lysate. All mice received three subcutaneous (sc) injections with $1 \times 10 \mathrm{e}^{6}$ pulsed DC (pDC) spaced one week, one group also received one it injection of $2 \times 10 \mathrm{e}^{5}$ $\mathrm{pDC}$ (it-sc). The combination of systemic and it vaccination cured $70 \%$ of the mice, whereas sc injection cured $50 \%$ of mice ( $P<0.0001$ and $P=0.002$ vs controls, respectively). To characterize the effect of the treatments on tumor microenviroment we used RT-PCR and found that TGF-beta expression is 1.4 fold higher in sc than it-sc $(P=0.04)$ and IFN-gamma expression twofold higher in it-sc than sc $(P=0.02)$. The analysis of regulatory T cells (Treg) by flow cytometry and RT-PCR on peripheral blood revealed a slight decrease in vaccinated compared to naïve mice. Histological evaluations revealed the persistence of viable $\mathrm{pDC}$ it and the interaction with tumor infiltrating lymphocytes, suggesting that DC can mediate biological activities in situ. Also of interest are in vitro observations that $\mathrm{pDC}$ produce higher level of IFN-gamma and TNF-alpha and lower level of IL-6 than unpulsed DC. Our results suggest that it DC can modulate tumor microenvironment and potentiate the anti-tumor immune response, exerting a dual function on tumor biology and immune suppression.

\section{P162}

\section{Bacterial ghost platform technology for DNA delivery}

\section{U. B. Mayr, W. Lubitz; \\ BIRD-C, Kritzendorf, Austria}

Bacterial Ghosts (BG) are non-living Gram-negative bacterial cell envelopes produced by controlled expression of lysis gene $\mathrm{E}$ from bacteriophage PhiX174. They are devoid of cytoplasmic contents and retain their natural outer surface make-up including bioadhesive properties. Therefore BG offer efficient targeting functions for protein antigens, mediators of the immune response and DNA to antigen presenting cells (APC) such as macrophages and dendritic cells (DC). The procedure of loading BG with DNA plas- mids is simple and the localisation of the external loaded DNA is in close association with the inner membrane of BG.

E. coli and M. haemolytica ghosts loaded with plasmid DNA encoding green fluorescent protein showed transfection efficiencies of $60 \%$ for murine macrophages, and $85 \%$ for human monocytederived DC. Intradermal and intramuscular immunisation studies of Balb/c mice with BG loaded with $\mathrm{pCMV} \beta$ DNA demonstrated efficient humoral and cellular immune responses against encoded $\beta$ galactocidase. As a direct consequence of the BG carrier used, the Thelper cell $(\mathrm{TH})$ response was modulated from a mixed $\mathrm{TH} 1 / \mathrm{TH} 2$ to a more dominant $\mathrm{TH} 2$ pattern. In model studies for potential human applications of the BG-DNA technology, intravenous immunisation of mice with autologous DC transfected ex vivo with pCMV $\beta$-loaded BG elicited antigen specific immune responses.

Recently, the multistep procedure of loading BG with DNA plasmids could be simplified into a one-step procedure with in vivo production of membrane-anchored minicircle DNA. The major advantage of the novel technology lies in the establishment of a simple GMP process for the production of minicircle DNA carried in $\mathrm{BG}$.

\section{Immune Stimulation}

\section{P163}

Impaired and inflammatory clearance lead to the initiation and perpetuation of chronic autoimmunity

\author{
U. S. Gaipl ${ }^{1,2}$, L. E. Munoz ${ }^{1}$, G. Grossmayer ${ }^{1}$, K. Sarter $^{1}$, \\ C. Schulze ${ }^{l}$, S. Franz ${ }^{1}$, M. Herrmann ${ }^{1}$; \\ ${ }^{1}$ Institute of Clinical Immunology and Rheumatology, Erlangen, \\ Germany, ${ }^{2}$ Department of Experimental Medicine I, Erlangen, \\ Germany
}

Systemic lupus erythematosus (SLE) is a very heterogeneous systemic autoimmune disease, in which autoantibody synthesis against nuclear constituents is the main immunological characteristic. We previously showed that in lymph nodes of some patients with SLE apoptotic material was not sufficiently cleared by tingible body macrophages. Instead, it was observed to be associated with the surfaces of follicular dendritic cells (FDC).

Chemotactic attraction signals of phagocytes for apoptotic cells exist. We analysed sera from SLE patients in migration assays and observed that the attraction signals of apoptotic cells were blocked by sera of approximately $30 \%$ of the SLE patients. Furthermore, macrophages differentiated from CD34 positive stem cells of the peripheral blood displayed anomalies in morphology and a reduced adherence and phagocytic capacity in the case of patients with SLE. However, whole blood assays showed that non-professional phagocytes from the peripheral blood (monocytes and granulocytes) took up necrotic nuclei much better than controls, which ignore this kind of "prey". This late phase clearance was strictly dependent on serum factors present only in the plasma of patients with SLE. The best candidates for this adjuvant activity for phagocytosis are autoantibodies binding to the surfaces of the dead cells.

We conclude that in SLE patients the retention of autoantigens on the surfaces of FDC as well as the presentation of nuclear autoantigens by dendritic cells may override the highly important mechanism of $\mathrm{B}$ and $\mathrm{T}$ cell tolerance, respectively. Additionally, autoantibody-mediated inflammatory clearance of certain nuclear targets contributes to the perpetuation of autoimmunity. 


\section{P164}

Endogenous annexinA5 influences the immune response to apoptotic and necrotic cells

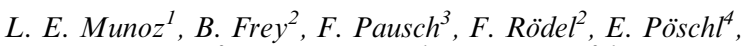
K. von der Mark ${ }^{3}$, M. Herrmann ${ }^{1}$, U. S. Gaipl ${ }^{3,1}$;

${ }^{1}$ Institute of Clinical Immunology and Rheumatology, Erlangen, Germany, ${ }^{2}$ Department of Radiooncology, Erlangen, Germany,

${ }^{3}$ Department of Experimental Medicine I, Erlangen, Germany,

${ }^{4}$ Biomedical Research Centre, University of East Anglia,

Norwich, UK

As the exposition of phosphatidylserine (PS) is a hallmark of apoptotic cells, the high affinity binding of labeled annexinA5 (AxA5) was extensively used for identifying apoptotic cells. In vivo, PS ensures a swift recognition and uptake by phagocytes of apoptotic and necrotic cells. AxA5 is a natural occurring highly specific ligand for PS and may interfere with the immunosuppressive effects of apoptotic cells.

Using AxA5-deficient mice we studied the influence of endogenous AxA5 on the allogeneic reaction against apoptotic and necrotic cells. AxA5-deficient mice showed no allogeneic reaction against cells necrotized by mechanical stress. In striking contrast, an allogeneic reaction was observed in WT mice. Furthermore, late apoptotic cells also led to a significantly higher delayed type hypersensitivity reaction in the case of WT animals. Very interestingly, AxA5-deficient macrophages had a higher immunosuppressive potential in vitro. We also injected subcutaneously allogeneic CT26 colorectal tumor cells and monitored the immune response. In WT animals a significantly faster decline in size of the allogeneic tumor was to be observed in comparison to AxA5-deficient mice.

We conclude that a disturbed PS-dependent clearance by macrophages of apoptotic cells leads to the accumulation of the latter and to the occurrence of late apoptotic/secondary necrotic cells. Danger signals are released and a pro-inflammatory microenvironment results. The described mechanism can be applied for efficient tumor vaccines. The immunity against allogeneic apoptotic and necrotic cells is increased in the presence of endogenous AxA5 and could be generally heightened by adding AxA5 to tumor cells in vaccination trials.

\section{P165}

\section{Coencapsulation of adjuvants and antigen into the same microspheres enables the generation of potent cytotoxic $T$ lymphocyte responses}

\author{
E. Schlosser ${ }^{1}$, M. Müller ${ }^{1}$, S. Fischer ${ }^{2}$, S. Basta ${ }^{1}$, D. H. Busch ${ }^{3}$, \\ B. Gander ${ }^{2}$, M. Groettrup ${ }^{1}$; \\ ${ }^{1}$ Division of Immunology, Konstanz, Germany, ${ }^{2}$ Institute of Phar- \\ maceutical Sciences, ETH Zürich, Zürich, Switzerland, ${ }^{3}$ Institute \\ for Medical Microbiology, Immunology, and Hygiene, Technical \\ University Munich, Munich, Germany
}

Immature dendritic cells phagocytose viruses or bacteria which leads to presentation of T cell epitopes on MHC class I and II molecules and simultaneously to their maturation triggered via tolllike receptors (TLR). Recently, it has been shown in vitro that antigens are presented on class II more efficiently, when a TLR ligand and an antigen are contained within the same particle. Here we addressed whether this finding can be extended to cross-priming of cytotoxic T lymphocytes (CTL) in vivo and exploited for the formulation of $\mathrm{T}$ cell vaccines. We found that the efficiency of crosspriming in mice after vaccination with biodegradable poly(D,Llactide-co-glycolide) (PLGA) microspheres (MS) was two fold en- hanced when ovalbumin was coencapsulated together with either $\mathrm{CpG}$ oligonucleotides as compared to co-inoculation of ovalbumin bearing MS with either soluble or separately encapsulated $\mathrm{CpG}$ oligonucleotides. A single immunization with $\mathrm{MS}$ containing $\mathrm{CpG}$ oligonucleotides and ovalbumin yielded $9 \%$ of SIINFEKL $/ \mathrm{H}-2 \mathrm{~Kb}$ tetramer positive CTLs, production of IFN- $\gamma$, efficient cytolysis of epitope charged target cells in vivo and in vitro, and full protection from recombinant vaccinia viruses. Taken together, coencapsulation of adjuvant and antigen into microparticles is an important paradigm for the design of vaccines and the generation of potent CTL responses. Furthermore we began to establish PLGA MS encapsulated LCMV derived nucleoprotein as a second antigen model. However, the aspired aim of this project is to develop a PLGA-MS based immunotherapy against prostate carcinoma using the TRAMP mouse, a well accepted transgenic mouse model in which adenocarcinomas develop within the prostate.

\section{P166}

Interleukin-15 liver gene transfer increases the number and function of IKDCs and NK cells

\author{
O. Murillo, A. Arina, A. Azpilicueta, I. Gabari, C. Alfaro, \\ J. L. Perez-Gracia, S. Hervás-Stubbs, I. J. Melero; \\ CIMA. Universidad de Navarra, 31008 Pamplona, Spain
}

An expression cassette encoding human IL-15 (hIL-15) has been transferred by hydrodynamic injection into the liver of mice, resulting in transient expression of the cytokine that peaks in plasma $8 \mathrm{~h}$ after injection and is detectable during the first 24-48 h. The fast decline of hIL-15 in serum seems to be the result of the rapid elimination of the plasmid DNA from the liver. Nonetheless, doses can be repeated in the same mouse achieving similar levels of circulating cytokine. hIL-15 hydrodynamic gene transfer results in an expansion of total NK cells (NK1.1 + CD3-) and the recently defined Interferon-producing killer dendritic cell (IKDC) subpopulation (NK1.1 + CD3CD11cintB220 + GR1-). The attained expansion of these leukocytes in the liver is more dramatic, although it can also be detected in the spleen. Numeric increases are at least in part the result of proliferation from already differentiated cells and are followed by enhanced cytolytic activity against YAC-1 cells and the surface expression of TRAIL and CD137. However, transient hIL-15 liver gene transfer by itself has no significant effects against various syngenic tumors with the exception of a beneficial effect on B16F10 melanoma liver metastasis. The novel link between IL-15 and IKDC further suggests potential for combination immunotherapy strategies.

\section{P167}

'The phosphatidylserine ligand annexin A5 heightens the immunogenicity of irradiated tumour cells in vivo'

\author{
C. Schulze ${ }^{1}$, K. Sarter ${ }^{1}$, B. Frey ${ }^{2}$, L. E. Munoz ${ }^{1}$, F. Pausch ${ }^{3}$, \\ M. Hermann ${ }^{1}$, U. S. Gaipl ${ }^{1,3}$; \\ ${ }^{1}$ Institute of Clinical Immunology and Rheumatology, Department \\ of Internal Medicine 3, University Hospital Erlangen, Erlangen, \\ Germany, ${ }^{2}$ Department of Radiooncology, Friedrich-Alexander- \\ University of Erlangen-Nuremberg, Erlangen, Germany, ${ }^{3}$ Depart- \\ ment of Experimental Medicine I, Nikolaus-Fiebiger-Centre of \\ Molecular Medicine, Friedrich-Alexander-University of Erlangen- \\ Nuremberg, Erlangen, Germany
}

Strategies to increase the immunogenicity of tumour cells are eagerly demanded. The immunization with irradiated lymphoma 
cells leads to a tumour specific immune response. However, the effectivity is low, since the immunogenic properties of apoptotic cells are poor because of their non- or even anti-inflammatory clearance. The protein annexinA5 (AxA5) binds phosphatidylserine (PS) of cell membranes with high affinity in vitro and in vivo and thereby interferes with the swift uptake by macrophages of PS-exposing apoptotic cells. We could show that the treatment of apoptotic human cells with AxA5 significantly increased their immunogenicity.

Here, we investigated the immunogenic properties of irradiated tumour cells. Mice were subcutaneously immunized with irradiated highly tumourigenic Rauscher virus-induced H-2 RMA lymphoma cells. The irradiated RMA cells were pre-incubated with or without AxA5. We could show that AxA5 conducted UVirradiated lymphoma cells to CD8 positive dendritic cells in vivo and proinflammatory cytokines were released. The immune response was specific as immunised mice were not able to antagonise not-related tumour cells. Besides, the immune response was provided with memory. Mice effectively rejected viable RMA cells 72 days after the immunisation. Most importantly, the injection of AxA5-coupled UV-irradiated tumour cells evoked a reduction of already formed tumours in the mouse.

We conclude that the availability of AxA5 at the site of clearance of dead tumour cells disturbs their PS-dependent recognition and removal und thus abrogates their immunosuppressive clearance. Consequently, adjuvants like AxA5 can be used for the effective immunological attack and clearance of growing tumour cells (e.g. metastases).

\section{P168}

\section{Hydrophobic portions of molecules; Damage-associated molecular pattern that initiates innate immune responses}

\author{
M. Bae, H. Moon, S. Lee, S. Seong; \\ Hyppo Lab., Department of Microbiology and Immunology, \\ Seoul National University College of Medicine, 28 Yeongeon-dong, \\ Jongno-gu, Seoul, Republic of Korea
}

It is currently thought that immune responses are initiated by pathogen-associated molecular patterns (PAMPs) or by damageassociated molecular patterns (DAMPs). However, these two groups of molecules might not be mutually exclusive. Many of them might be part of an evolutionarily ancient alert system, in which the hydrophobic portions (Hyppos) of biological molecules, when exposed, act as universal DMAPs to initiate repair and immunity. Since Hyppos are normally hidden from the aqueous environment and exposed Hyppos rapidly form non-productive or even toxic aggregates, organisms expend a great deal of metabolic energy to prevent such exposure and maintain homeostasis. Therefore, when cells die necrotically, a number of Hyppos released into its immediate environment may act as danger signals or DAMPs.

We report here that necrotic cells (NCs) can induce activation of dendritic cells (DCs) via Toll like receptor 4 (TLR4) and TLR4 recognizes DAMPs as much as PAMPs. Compared with DCs from wild type mice, those from TLR4-/- mice were less responsive to NCs in induction of costimulatory molecule, IL-12p40 secretion, and naïve $\mathrm{T}$ cell activation. Microarray analysis revealed that NCs can also trigger chemokine expression in DCs via TLR4. The DC activation by NCs was inhibited by apolipoprotein. In addition, we identified cardiolipin, a major component of inner membrane of mitochondria and structurally similar to lipid A of LPS, as an additional DAMP capable of activating DCs. Therefore, we suggest that Hyppos from NCs are evolutionarily ancient DAMPs and TLR4 is involved in DC activation by Hyppos.

\section{P169}

A novel role of complement factor $\mathrm{C1q}$ in augmenting the presentation of antigen captured in immune complexes to CD8 + T lymphocytes

N. van Montfoort, J. de Jong, D. Schuurhuis, E. van der Voort, M. Camps, C. van Kooten, M. Daha, T. Huizinga, S. Verbeek, R. Toes, F. Ossendorp;

Leiden University Medical Center, Leiden, The Netherlands

Antigen-antibody immune complexes (IC) are naturally occurring antigen complexes that bind with high affinity to Fc receptors on the surface of dendritic cells (DC). Fc receptor binding facilitates both highly efficient uptake of antigen and maturation of DC. The matured DC can effectively process and cross-present antigenic peptide, derived from the antigen captured in IC, in MHC class I and can induce strong CTL responses in vivo.

Here, we studied the role of different $\mathrm{Fc} \gamma \mathrm{R}$ and complement components in cross-presentation of i.v. administered OVA antigen captured in IC to OVA-specific CD8 $+\mathrm{T}$ cells in genetically modified mice deficient for $\mathrm{F} c \gamma \mathrm{R}$ and complement components. Cross-presentation was partly reduced in FcR $\gamma$-chain-deficient mice, but not affected in Fc $\gamma \mathrm{RI} / \mathrm{II} / \mathrm{III}$-deficient mice, complement factor C3-deficient mice or $\mathrm{Fc} \gamma \mathrm{RI} / \mathrm{II} / \mathrm{IIIxC} 3$-deficient mice. Importantly, cross-presentation was significantly reduced in mice deficient for C1q. CD8 $+\mathrm{T}$ cell responses could be restored in these mice when IC were incubated with purified $\mathrm{Clq}$ before injection. In addition, in vivo uptake of OVA-IC in CD11c + cells in the spleen was significantly reduced in $\mathrm{Clq}$ deficient mice compared to wt mice. In vitro studies showed that purified $\mathrm{Clq}$ could strongly enhance the uptake of IC and antigen presentation in MHC class I by cultured DC. Heat inactivation abrogated the Clq-mediated IC uptake and antigen presentation. Together, these results indicate a novel function of $\mathrm{Clq}$, which is present in high levels in the bloodstream, by directly enhancing the uptake and MHC class I presentation of antigen captured in IC by APC to $\mathrm{CD} 8+\mathrm{T}$ cells.

\section{P170}

\section{Dendritic cell differentiation state and their interaction with NKT cells determines Th1/Th2 differentiation in the murine model of Leishmania major infection}

\author{
C. Wiethe ${ }^{1}$, A. Debus ${ }^{2}$, A. Steinkasserer ${ }^{1}$, M. B. Lutz $^{3}$, \\ A. Gessner ${ }^{2}$; \\ ${ }^{1}$ Department of Dermatology, Erlangen, Germany, ${ }^{2}$ Clinical \\ Microbiology, Immunology and Hygiene, Erlangen, Germany, \\ ${ }^{3}$ Department of Virology and Immunology, Würzburg, Germany
}

Recent reports demonstrated that dendritic cells (DC) sense inflammatory and microbial signals differently, redefining their classical subdivision into an immature endocytotic and a mature antigen-presenting differentiation stage. While both signals induce DC maturation by upregulating MHC II and costimulatory molecules, only Toll-like receptor signals are able to trigger proinflammatory cytokine secretion by DC, including Th1-polarising IL-12.

Here, we explored the murine Leishmania major infection model to examine the $\mathrm{CD} 4{ }^{+} \mathrm{T}$ cell response induced by differentially 
matured DC. Initial experiments showed that pulsing DC with $L$. major lysate did not affect DC maturation with $\mathrm{TNF} \alpha$ or LPS + anti-CD40. When partially matured TNF-DC were injected into $\mathrm{Balb} / \mathrm{c}$ mice prior to infection, the mice failed to control L. major infection and developed a Th2 response which was dependent on IL- $4 \mathrm{R} \alpha$ signaling. In contrast, injections of fully matured LPS + CD40-DC induced a Th1 response controlling the infection. Furthermore, when the expression of different Notch ligands on DC was analyzed, we found increased expression of Th2-promoting Jagged 2 in TNF-DC whereas LPS + CD40-DC upregulated the Th1-inducing Delta4 and Jagged 1 molecules. The Th2 polarization induced by TNF-DC required interaction with CD1d-restricted NKT cells. However, NKT cell activation by L. major lysatepulsed DC was not affected by blockade of the endogenous glycolipid suggesting exchange with exogenous parasite-derived CD1 glycolipid antigen.

In sum, the differentiation stage of $\mathrm{DC}$ as well as their interaction with NKT cell determines Th1/Th2 differentiation. These results have generic implications for the understanding of DC instructed Th cell responses and the development of improved DC vaccines against leishmaniasis.

\section{P171}

\section{Targeting chemoresistant cancer stem cells using tumor-specific $\mathbf{T}$ cells}

A. M. Leen, J. Shafer, A. Lu, M. K. Brenner, C. M. Rooney, C. M. Bollard, A. E. Foster;
Center for Cell and Gene Therapy, Baylor College of Medicine, Texas Children's Hospital, Houston, TX, USA

Resistance of cancer stem cells to chemotherapy has been attributed to the expression of multidrug-resistance pumps, such as MDR-1 and ABCG2, that actively efflux chemotherapeutic agents preventing their intracellular accumulation and cell death. This subpopulation likely contributes to disease relapse and can be indentified by flow cytometry using Hoechst dye, where the cells appear as a distinct "side-population" (SP). We hypothesized that if target antigens for immunotherapy could be identified on these cancer stem cells, then a combination of chemotherapy and cell therapy might improve long term tumorfree survival. To test this hypothesis in Hodgkin disease (HD), we evaluated $5 \mathrm{HD}$ cell lines for the presence of SP cells and identified 2 HD cell lines (HDLM2 and L428) with distinct SP populations (frequency $0.8-2 \%$ ). In contrast, the 3 HD cell lines lacking SP cells showed chemosensitivity. To identify potential immunotherapeutic target antigens expressed in the SP populations, we screened SP and non-SP cells for cancer testis antigens (CTA) and found expression of SSX2, MAGE-A4, and NY-ESO. We then developed protocols for the activation and expansion of cytotoxic T lymphocytes (CTL) targeting these CTAs. By stimulating CD8 $+\mathrm{T}$ cells with dendritic cells pulsed with an overlapping peptide library spanning the SSX2 protein in the presence of IL7, IL12, and IL15, we successfully generated SSX2-specific CTL that recognize SSX2-expressing targets. If CTA-specific CTL can specifically target the chemoresistant SP population, novel adjuvant strategies could be developed using combined chemo- and immuno-therapies to improve outcomes for patients with HD. 